\title{
SINGULAR PERTURBATION IN A NONLOCAL DIFFUSION PROBLEM
}

\author{
CLAUDIA LEDERMAN AND NOEMI WOLANSKI
}

\begin{abstract}
We study a singular perturbation problem for a nonlocal evolution operator. The problem appears in the analysis of the propagation of flames in the high activation energy limit, when admitting nonlocal effects.

We obtain uniform estimates and we show that, under suitable assumptions, limits are solutions to a free boundary problem in a viscosity sense and in a pointwise sense at regular free boundary points.

We study the nonlocal problem both for a single equation and for a system of two equations.

Some of the results obtained are new even when the operator under consideration is the heat operator.
\end{abstract}

\section{INTRODUCTION}

The reaction-diffusion equation with ignition term

$$
\Delta u-u_{t}=\beta(u) \quad \text { in } \mathbb{R}^{N} \times(0,+\infty),
$$

arises in combustion theory to describe the propagation of curved premixed equi-diffusional deflagration flames. Here $\beta$ is assumed to be a Lipschitz continuous function, support $\beta=[0,1]$ and $\beta>0$ in $(0,1)$. The function $u$ represents the normalized temperature of the mixture -more precisely, $u=\lambda\left(T_{f}-T\right)$, with $T_{f}$ the flame temperature and $\lambda$ a normalization factor- or $u$ may be seen as the concentration of the reactant, so it is in general assumed to be nonnegative.

It is of particular interest the high activation energy analysis for this model. Namely, the study of equation (1.1) for small values of a parameter involved in the model - the inverse of the activation energy. In this case, the problem under study takes the form

$$
\Delta u^{\varepsilon}-u_{t}^{\varepsilon}=\beta_{\varepsilon}\left(u^{\varepsilon}\right) \quad \text { in } \mathbb{R}^{N} \times(0,+\infty),
$$

with $u^{\varepsilon} \geq 0, \beta_{\varepsilon}(s)=\frac{1}{\varepsilon} \beta\left(\frac{s}{\varepsilon}\right), \beta$ as above, and $\varepsilon>0$ small. For the derivation of the model we refer to [3], [17].

The study of the limit $\varepsilon \rightarrow 0$ was proposed in the 1930s in [19] and has been much discussed in the literature in the last years, starting with the pioneering works by Berestycki, Caffarelli and Nirenberg [2] and by Caffarelli and Vazquez [9]. It has been shown, under certain assumptions, that it is possible to pass to the limit in (1.2) and that the limit function $u$ is a solution to the

1991 Mathematics Subject Classification. 35R35, 35K60, 80A25.

Key words and phrases. Free boundary problem, viscosity solutions, nonlocal diffusion, nonlocal evolution operator, combustion.

Departamento de Matemática, Facultad de Ciencias Exactas y Naturales, Universidad de Buenos Aires, (1428) Buenos Aires, Argentina, clederma@dm.uba.ar, wolanski@dm.uba.ar.

The research of the authors was partially supported by Fundación Antorchas Project 13900-5, ANPCyT PICT No. 03-05009 and CONICET PIP0660/98. The authors are members of CONICET . 
free boundary problem

$$
\begin{array}{ll}
\Delta u-u_{t}=0 & \text { in }\{u>0\} \\
|\nabla u|^{2}=2 M & \text { on } \partial\{u>0\}
\end{array}
$$

in some appropriate sense. Here $\int \beta(s) d s=M$. For the precise results we refer to $[2,6,7,8,9$, $10,15,18]$.

In the recent work [4], the following nonlocal problem was considered:

$$
\theta \Delta u+(1-\theta)(J * u-u)-u_{t}=\beta(u) \text { in } \mathbb{R}^{N} \times(0,+\infty),
$$

with $0 \leq \theta \leq 1, \beta$ as above and $J=J(x)$ an even nonnegative kernel with unit integral (the symbol $*$ denoting spatial convolution).

The motivation for considering the nonlocal problem (1.4) lies in the fact that the linear operator $A$ defined by $A u=J * u-u$ appears as a natural nonlocal counterpart of the Laplacian, sharing with it some properties, such as a form of maximum principle. On the other hand, as $J$ has unit integral, the operator $A u$ has the alternative form

$$
A u(x)=\int J(x-y)(u(y)-u(x)) d y .
$$

The integral $\int J(x-y) u(y) d y$ may be seen as the proportion of reactant that migrates to the point $x$ from all points $y$ in space due to diffusion and $\int J(x-y) u(x) d y$ as the proportion of reactant that migrates from $x$ to all other points $y$ in space. So that $A u(x)$ represents the variation of concentration of reactant at the point $x$ due to diffusion.

These facts, among others, have been considered by several authors in different contexts in order to replace the Laplacian by the operator $A$ in the modelling of reaction diffusion problems (see [11] and [16] for excellent surveys on nonlocal reaction diffusion models).

Returning to the model under consideration, in [4] the author studies the one dimensional travelling wave solutions of the nonlocal problem (1.4), obtaining the same type of results that were known for the combustion model (1.1).

So a natural question is: what happens with the high activation energy analysis for the nonlocal combustion model (1.4), namely, with the nonlocal singular perturbation problem

$$
\theta \Delta u^{\varepsilon}+(1-\theta)\left(J * u^{\varepsilon}-u^{\varepsilon}\right)-u_{t}^{\varepsilon}=\beta_{\varepsilon}\left(u^{\varepsilon}\right) \quad \text { in } \mathbb{R}^{N} \times(0,+\infty),
$$

as $\varepsilon \rightarrow 0$ ? More precisely, is it possible to pass to the limit? Is the limit problem a free boundary problem? In that case, is it possible to obtain for problem (1.5) the same type of results that are known for problem (1.2), as $\varepsilon \rightarrow 0$ ? Moreover, what is the interaction between the nonlocal term and the free boundary condition of the limit problem?

As a first step towards the understanding of the nonlocal singular perturbation problem (1.5), we would like to point out that given a family $v^{\varepsilon}$ of nonnegative solutions to problem (1.2), and given $0<\theta \leq 1$, the rescaled functions

$$
u^{\varepsilon}(x, t)=v^{\varepsilon}\left(\frac{x}{\sqrt{\theta}}, t\right)
$$

are solutions to

$$
\theta \Delta u^{\varepsilon}+(1-\theta)\left(J * u^{\varepsilon}-u^{\varepsilon}\right)-u_{t}^{\varepsilon}=\beta_{\varepsilon}\left(u^{\varepsilon}\right)+g^{\varepsilon} \quad \text { in } \mathbb{R}^{N} \times(0,+\infty),
$$

with

$$
g^{\varepsilon}=(1-\theta)\left(J * u^{\varepsilon}-u^{\varepsilon}\right) .
$$


Thus, such a family provides us with an example of solution to a problem of type (1.5), with a forcing term $g^{\varepsilon}$ appearing in the right hand side. This is an example in which we know the answers to the questions posed above. In particular, the limit problem is a free boundary problem and the free boundary condition when $\Delta u$ is replaced by $\theta \Delta u+(1-\theta)(J * u-u)$ becomes $\theta|\nabla u|^{2}=2 M$ instead of $|\nabla u|^{2}=2 M$.

So a natural question now is: what happens with the nonlocal problem (1.6) when we have an arbitrary forcing term $g^{\varepsilon}$, not of the type (1.7)? What happens with the nonlocal problem (1.6) when we have in particular $g^{\varepsilon} \equiv 0$ ?

\section{Some conclusions}

In this paper we prove existence and uniqueness of solutions to

$$
\begin{aligned}
\theta \Delta u^{\varepsilon}+(1-\theta)\left(J * u^{\varepsilon}-u^{\varepsilon}\right)-u_{t}^{\varepsilon} & =\beta_{\varepsilon}\left(u^{\varepsilon}\right)+g^{\varepsilon} \quad \text { in } \mathbb{R}^{N} \times(0,+\infty), \\
u^{\varepsilon}(x, 0) & =u_{0}^{\varepsilon}(x) \quad \text { in } \mathbb{R}^{N},
\end{aligned}
$$

where $0<\theta \leq 1, g^{\varepsilon}$ are given functions, and $\beta_{\varepsilon}$ and $J$ are as above. We obtain uniform estimates and conditions under which $u^{\varepsilon}$ are nonnegative. When $u^{\varepsilon} \geq 0$ we show that we can pass to the limit $(\varepsilon \rightarrow 0)$ and that, under suitable assumptions, the limit function $u$ is a solution to the following free boundary problem

$$
\begin{aligned}
\theta \Delta u+(1-\theta)(J * u-u)-u_{t} & =g & & \text { in }\{u>0\} \\
\theta|\nabla u|^{2} & =2 M & & \text { on } \partial\{u>0\}
\end{aligned}
$$

in a viscosity sense and in a pointwise sense at regular free boundary points. Here $g=\lim g^{\varepsilon}$ and $\int \beta(s) d s=M$.

In particular, for any forcing term $g^{\varepsilon}$ we obtain, under suitable assumptions, the same answers as in the case when $g^{\varepsilon}$ is the one in (1.7). This is, the limit problem of (1.8) is a free boundary problem and the free boundary condition when $\Delta u$ is replaced by $\theta \Delta u+(1-\theta)(J * u-u)$ becomes $\theta|\nabla u|^{2}=2 M$ instead of $|\nabla u|^{2}=2 M$.

We thus notice that, although the nonlocal operator $A u=J * u-u$ shares some properties with the Laplacian, it plays a completely different role in this context.

It is also worth observing that, when we pass to the limit $\varepsilon \rightarrow 0$ in (1.8), we obtain a free boundary problem where the jump of the gradient on the free boundary, namely $\sqrt{\frac{2 M}{\theta}}$, becomes unbounded as $\theta \rightarrow 0$. This is in contrast with the situation observed in the analysis performed for problem (1.5) with $\varepsilon$ fixed in [4], where the relevant magnitudes, such as the speed of the travelling waves, remain bounded as $\theta \rightarrow 0$.

In the process of studying the problem posed above we dealt with new and interesting questions for related problems that we describe below.

\section{Main results and outline of the paper}

We start the paper by proving in Section 2 some preliminary results about the nonlocal problem (1.8), $\varepsilon$ fixed, such as existence, uniqueness and comparison of solutions.

Then we have that, under suitable assumptions, the solutions $u^{\varepsilon}$ of (1.8) are nonnegative and uniformly bounded in $L^{\infty}$ norm and therefore the functions

$$
\bar{u}^{\varepsilon}(x, t)=u^{\varepsilon}(\sqrt{\theta} x, t)
$$

are nonnegative, uniformly bounded solutions to

$$
\left(P_{\varepsilon}\left(f^{\varepsilon}\right)\right) \quad \Delta \bar{u}^{\varepsilon}-\bar{u}_{t}^{\varepsilon}=\beta_{\varepsilon}\left(\bar{u}^{\varepsilon}\right)+f^{\varepsilon}
$$


with

$$
f^{\varepsilon}(x, t)=-(1-\theta)\left(J * u^{\varepsilon}-u^{\varepsilon}\right)(\sqrt{\theta} x, t)+g^{\varepsilon}(\sqrt{\theta} x, t)
$$

uniformly bounded.

Thus, the secondary goal of this paper is the study of problem $P_{\varepsilon}\left(f^{\varepsilon}\right)$ - that had only been studied when $f^{\varepsilon} \equiv 0$. When $f^{\varepsilon} \not \equiv 0$ we encountered interesting new fenomena that can be seen, in particular, in the examples in Section 3. Namely, we found bounded global stationary solutions (Figures 2 and 4), periodic stationary solutions (Figure 2) and stationary solutions with limits (as $\varepsilon \rightarrow 0)$ that degenerate (Figure 5).

This last example shows that there exist limits $u($ as $\varepsilon \rightarrow 0)$ of solutions $u^{\varepsilon}$ to $P_{\varepsilon}\left(f^{\varepsilon}\right)$ which degenerate on $\partial\{u>0\}$ even when these limits have smooth $\partial\{u>0\}$. This cannot happen if $f^{\varepsilon} \equiv 0$ by Hopf's Principle since, in that case, the limits $u$ are caloric functions in $\{u>0\}$.

In particular, this example shows that we cannot expect the free boundary condition $|\nabla u|^{2}=$ $2 M$ to hold for any limit $u$ - not even in the stationary case and with $\partial\{u>0\}$ smooth.

Section 3 also contains examples of situations with $f^{\varepsilon} \equiv 0$ that had not been observed previously in the literature (see Prop. 3.2 and Figure 1).

In Sections 4 to 8 we consider a family $u^{\varepsilon}$ of solutions to $P_{\varepsilon}\left(f^{\varepsilon}\right)$ in a domain $\mathcal{D} \subset \mathbb{R}^{N+1}$ such that $u^{\varepsilon}$ and $f^{\varepsilon}$ are uniformly bounded in $L^{\infty}(\mathcal{D})$. First we obtain further uniform estimates that allow the passage to the limit of $u^{\varepsilon}$ as $\varepsilon \rightarrow 0$, analyze some basic limits and study the behavior of limit functions $u$ near $\partial\{u>0\}$. Then, in Sections 7 and 8 we prove that, under suitable assumptions, a limit $u$ of solutions $u^{\varepsilon}$ of $P_{\varepsilon}\left(f^{\varepsilon}\right)$ is a solution (in some appropriate sense) of the free boundary problem

$$
\begin{array}{ll}
\Delta u-u_{t}=f & \text { in }\{u>0\} \\
|\nabla u|=\sqrt{2 M} & \text { on } \partial\{u>0\}
\end{array}
$$

where $f=\lim f^{\varepsilon}$.

In fact, in Section 7 we prove that the free boundary condition $|\nabla u|=\sqrt{2 M}$ is satisfied (in the sense of an asymptotic development) at any point $\left(x_{0}, t_{0}\right) \in \partial\{u>0\}$ where the free boundary has an interior unit spatial normal in the parabolic measure theoretic sense. The result holds if $u$ satisfies a nondegeneracy assumption at the point. This is done in Theorem 7.1 , that is new even when $f^{\varepsilon} \equiv 0$. The nondegeneracy assumption is necessary as the examples in Section 3 show. On the other hand, Theorem 6.2 gives conditions under which the limit does not degenerate at a free boundary point.

In particular we find, when $f \leq 0$, that just some regularity of the free boundary at a free boundary point is enough to get the free boundary condition at the point (Corollary 7.1). The fact that the free boundary condition is satisfied if the free boundary is smooth at the point, without any further condition, is also new even when $f^{\varepsilon} \equiv 0$.

Notice that the sign condition on $f$ in Corollary 7.1 is not necessary to get the free boundary condition as example 3) in Proposition 3.4 shows.

In Section 8 we prove results on asymptotic developments at free boundary points in which there is a tangent ball contained either in $\{u>0\}$ or in $\{u \equiv 0\}$, that are new even when $f^{\varepsilon} \equiv 0$ (Theorems 8.1 and 8.2). As a corollary we prove that limits $u$ of solutions to $P_{\varepsilon}\left(f^{\varepsilon}\right)$ that satisfy a nondegeneracy assumption are viscosity solutions to $P(f)$ (Corollaries 8.1 and 8.2). The comments made above regarding the nondegeneracy assumption of Section 7 apply also to this section.

For the understanding of general limits, without any assumption, we refer to the results in Proposition 4.1 and Theorem 6.1. 
Then, in Section 9 we return to the nonlocal problem. In fact, we consider a family $u^{\varepsilon}$ of solutions to the nonlocal problem (1.8) and we apply the results of Sections 4 to 8 to the functions $\bar{u}^{\varepsilon}$ defined in (1.10) — that satisfy $P_{\varepsilon}\left(f^{\varepsilon}\right)$ with $f^{\varepsilon}$ defined in (1.11).

Moreover, we show that when $g^{\varepsilon} \equiv 0$ - as is the case in our original problem (1.5) - the forcing term $f=\lim f^{\varepsilon}$ in the equation for $\bar{u}=\lim \bar{u}^{\varepsilon}$ has some particular properties. Indeed, $f<0$ at any free boundary point. This rules out the possibility of degeneracy of $\bar{u}$ at the free boundary if the free boundary has some smoothness and thus, the free boundary condition in (1.9) is satisfied. The same conclusion holds if $g \leq 0$ (see Corollary 9.1).

In Section 9 we also discuss examples of solutions of the nonlocal problem (1.8). On one hand, we show that, under suitable assumptions, if $u=\lim u^{\varepsilon}$ has bounded initial support, then the support (and thus the free boundary) remains bounded and moreover, it shrinks. On the other hand, we find examples of choices of data $u_{0}^{\varepsilon}$ and $g^{\varepsilon}$ such that the solutions of the nonlocal problem (1.8) satisfy that $\bar{u}^{\varepsilon}$ meet the hypotheses of Theorem 9.3 , so that $\bar{u}$ is a viscosity solution to $P(f)$ in $\mathbb{R}^{N} \times(0, T)$.

Finally, in Section 10 we discuss the general model in the equidiffusional case that consists of a system of two nonlocal reaction diffusion equations. This system reduces to (1.8) when the initial condition for both unknowns as well as the forcing terms of both equations coincide, and was studied in the case of heat operators without forcing terms in [10]. Our results are new even in the case considered in [10]. The system in [10] appears in combustion to model the propagation of flames when Lewis number is 1 .

We include an Appendix where we prove general results on asymptotic developments for positive caloric functions. These developments are crucial for the proofs in Section 8. We believe that the results in the Appendix are of independent interest.

\section{Notation and assumptions}

Throughout the paper $N$ will denote the spatial dimension and, in addition, the following notation will be used:

For any $x_{0} \in \mathbb{R}^{N}, t_{0} \in \mathbb{R}$ and $\tau>0$

$$
\begin{array}{ll}
B_{\tau}\left(x_{0}\right):=\left\{x \in \mathbb{R}^{N} /\left|x-x_{0}\right|<\tau\right\}, & B_{\tau}\left(x_{0}, t_{0}\right):=\left\{(x, t) \in \mathbb{R}^{N+1} /\left|x-x_{0}\right|^{2}+\left|t-t_{0}\right|^{2}<\tau^{2}\right\}, \\
Q_{\tau}^{-}\left(x_{0}, t_{0}\right):=B_{\tau}\left(x_{0}\right) \times\left(t_{0}-\tau^{2}, t_{0}\right], & Q_{\tau}\left(x_{0}, t_{0}\right):=B_{\tau}\left(x_{0}\right) \times\left(t_{0}-\tau^{2}, t_{0}+\tau^{2}\right),
\end{array}
$$

and for any set $K \subset \mathbb{R}^{N+1}$

$$
\begin{aligned}
& \mathcal{N}_{\tau}(K):=\left\{(x, t) /(x, t) \in Q_{\tau}\left(x_{0}, t_{0}\right) \text { for some }\left(x_{0}, t_{0}\right) \in K\right\} \\
& \mathcal{N}_{\tau}^{-}(K):=\left\{(x, t) /(x, t) \in Q_{\tau}^{-}\left(x_{0}, t_{0}\right) \text { for some }\left(x_{0}, t_{0}\right) \in K\right\}
\end{aligned}
$$

When necessary, we will denote points in $\mathbb{R}^{N}$ by $x=\left(x_{1}, x^{\prime}\right)$, with $x^{\prime} \in \mathbb{R}^{N-1}$. Also, $\langle\cdot, \cdot\rangle$ will mean the usual scalar product in $\mathbb{R}^{N}$. Given a function $v$, we will denote $v^{+}=\max (v, 0)$, $v^{-}=\max (-v, 0)$. In addition, the symbols $\Delta$ and $\nabla$ will denote the corresponding operators in the space variables; the symbol $\partial_{p}$ will denote parabolic boundary.

We will say that a function $v$ is in the class $\operatorname{Lip}_{\text {loc }}(1,1 / 2)$ in a domain $\mathcal{D} \in \mathbb{R}^{N+1}$, if for every $\mathcal{D}^{\prime} \subset \subset \mathcal{D}$, there exists a constant $L=L\left(\mathcal{D}^{\prime}\right)$ such that

$$
|v(x, t)-v(y, \tau)| \leq L\left(|x-y|+|t-\tau|^{\frac{1}{2}}\right)
$$

for every $(x, t),(y, \tau)$ in $\mathcal{D}^{\prime}$. If the constant $L$ above does not depend on the set $\mathcal{D}^{\prime}$, we will say that $v \in \operatorname{Lip}(1,1 / 2)$ in $\mathcal{D}$.

We assume that the functions $\beta_{\varepsilon}$ are defined by scaling of a function $\beta: \mathbb{R} \rightarrow \mathbb{R}$ satisfying:

i) $\beta$ is a Lipschitz continuous function, 
ii) $\beta>0$ in $(0,1)$ and $\beta \equiv 0$ otherwise,

iii) $\int_{0}^{1} \beta(s) d s=M$.

And we define $\beta_{\varepsilon}(s)=\frac{1}{\varepsilon} \beta\left(\frac{s}{\varepsilon}\right)$ and $B_{\varepsilon}(s)=\int_{0}^{s} \beta_{\varepsilon}(\tau) d \tau$. On the kernel $J$ we assume:

i) $J(x)=J(-x)$ in $\mathbb{R}^{N}$,

ii) $J(x) \geq 0$ in $\mathbb{R}^{N}$,

iii) $\int_{\mathbb{R}^{N}} J(x) d x=1$.

The symbol $*$ will denote spatial convolution and $\theta$ will be a fixed constant, $0<\theta \leq 1$.

Finally, throughout the paper when we refer to the nonlocal problem we will mean that the operator $J * u-u$ appears in the equation.

\section{Preliminary Results}

In this section we prove some preliminary results about the nonlocal problem (1.8), $\varepsilon$ fixed, such as existence, uniqueness and comparison of solutions, that will be used in the paper.

Throughout the section we assume that the kernel $J$ is as in Section 1.

Proposition 2.1. Let $u_{0}^{\varepsilon} \in C\left(\mathbb{R}^{N}\right) \cap L^{\infty}\left(\mathbb{R}^{N}\right), g^{\varepsilon} \in L^{\infty}\left(\mathbb{R}^{N} \times\left(0, T_{1}\right)\right)$ and let $\beta_{\varepsilon}$ be as in Section 1. There exists $T_{0}=T_{0}\left(\left\|u_{0}^{\varepsilon}\right\|_{L^{\infty}\left(\mathbb{R}^{N}\right)},\left\|g^{\varepsilon}\right\|_{L^{\infty}\left(\mathbb{R}^{N} \times\left(0, T_{1}\right)\right)},\left\|\beta_{\varepsilon}^{\prime}\right\|_{L^{\infty}(\mathbb{R})}\right), T_{0} \leq T_{1}$, such that the problem

$$
\left\{\begin{aligned}
\theta \Delta u^{\varepsilon}+(1-\theta)\left(J * u^{\varepsilon}-u^{\varepsilon}\right)-u_{t}^{\varepsilon} & =\beta_{\varepsilon}\left(u^{\varepsilon}\right)+g^{\varepsilon} & \text { in } \mathbb{R}^{N} \times\left(0, T_{0}\right), \\
u^{\varepsilon}(x, 0) & =u_{0}^{\varepsilon}(x) & \text { in } \mathbb{R}^{N},
\end{aligned}\right.
$$

has a unique solution $u^{\varepsilon} \in C\left(\mathbb{R}^{N} \times\left[0, T_{0}\right]\right) \cap L^{\infty}\left(\mathbb{R}^{N} \times\left(0, T_{0}\right)\right)$.

Proof. We will proceed by a fixed point argument. In fact, let $M$ be such that $\left\|u_{0}^{\varepsilon}\right\|_{L^{\infty}\left(\mathbb{R}^{N}\right)} \leq M$. For $0<T \leq T_{1}$ let

$$
X=\left\{u \in C\left(\mathbb{R}^{N} \times[0, T]\right) /\|u\|_{L^{\infty}\left(\mathbb{R}^{N} \times(0, T)\right)} \leq 2 M\right\} .
$$

Next, let $v \in X$ be defined by $\mathcal{T}(v)=u$, with $u \in C\left(\mathbb{R}^{N} \times[0, T]\right) \cap L^{\infty}\left(\mathbb{R}^{N} \times(0, T)\right)$ the unique solution to

$$
\left\{\begin{aligned}
\theta \Delta u-u_{t} & =-(1-\theta)(J * v-v)+\beta_{\varepsilon}(v)+g^{\varepsilon} & \text { in } \mathbb{R}^{N} \times(0, T), \\
u(x, 0) & =u_{0}^{\varepsilon}(x) & \text { in } \mathbb{R}^{N} .
\end{aligned}\right.
$$

Then, there holds that $\mathcal{T}$ is a contraction in $X$ if $T$ is small enough.

Proposition 2.2. Let $u, v \in C\left(\mathbb{R}^{N} \times[0, T]\right) \cap C^{2,1}\left(\mathbb{R}^{N} \times(0, T]\right)$, $u$ and $v$ bounded, $u$ a subsolution and $v$ a supersolution to

$$
\theta \Delta w+(1-\theta)(J * w-w)-w_{t}=h(w)+g \quad \text { in } \mathbb{R}^{N} \times(0, T),
$$

with $h \in \operatorname{Lip}(\mathbb{R})$ and $g \in L^{\infty}\left(\mathbb{R}^{N} \times(0, T)\right)$. Assume that $u(x, 0) \leq v(x, 0)$ in $\mathbb{R}^{N}$. Then $u \leq v$ in $\mathbb{R}^{N} \times[0, T]$.

Proof. Step $I$. We will first show that if $U \in C\left(\mathbb{R}^{N} \times[0, T]\right) \cap C^{2,1}\left(\mathbb{R}^{N} \times(0, T]\right), U$ bounded, is such that

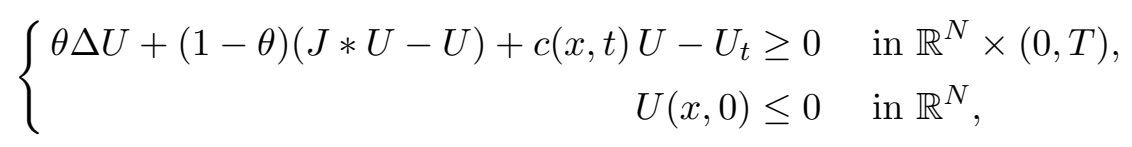


with $c \in L^{\infty}\left(\mathbb{R}^{N} \times(0, T)\right), c \leq 0$, then $U \leq 0$ in $\mathbb{R}^{N} \times[0, T]$. In order to prove this, let us first consider a family of kernels $J_{\delta}$ with compact support satisfying

$$
J_{\delta}(x)=J_{\delta}(-x), \quad J_{\delta}(x) \geq 0, \quad \int_{\mathbb{R}^{N}} J_{\delta}(x) d x=1, \quad J_{\delta} \rightarrow J \quad \text { in } \quad L^{1}\left(\mathbb{R}^{N}\right)(\delta \rightarrow 0),
$$

and observe that there holds

$$
\left(J_{\delta} *|y|^{2}\right)(x)-|x|^{2}=\int_{y_{1}>0} J_{\delta}(y)\left(|x-y|^{2}+|x+y|^{2}-2|x|^{2}\right) d y=2 \int_{y_{1}>0} J_{\delta}(y)|y|^{2} d y=C_{\delta} .
$$

Now let $A=\|U\|_{L^{\infty}\left(\mathbb{R}^{N} \times(0, T)\right)}$ and define, for $R>0$ and $\varepsilon>0$, the function $W=U-\frac{2 A N}{R^{2}}\left(\frac{|x|^{2}}{2 N}+\right.$ $\theta t)-\varepsilon t$. Let $\mathcal{L}_{\delta}$ be the operator in $(2.1)$, with $J$ replaced by $J_{\delta}$. We have

$$
\begin{aligned}
\mathcal{L}_{\delta} W & =\left(\theta \Delta U+(1-\theta)(J * U-U)+c U-U_{t}\right)+(1-\theta)\left(J_{\delta}-J\right) * U-(1-\theta) \frac{A}{R^{2}} C_{\delta} \\
& -c \frac{2 A N}{R^{2}}\left(\frac{|x|^{2}}{2 N}+\theta t\right)-c \varepsilon t+\varepsilon \geq-A\left\|J_{\delta}-J\right\|_{L^{1}\left(\mathbb{R}^{N}\right)}-\frac{A}{R^{2}} C_{\delta}+\varepsilon \geq \varepsilon / 2,
\end{aligned}
$$

where the last inequality holds if we first choose $\delta$ small enough so that $A\left\|J_{\delta}-J\right\|_{L^{1}\left(\mathbb{R}^{N}\right)} \leq \varepsilon / 4$ and then choose $R$ big enough so that $\frac{A}{R^{2}} C_{\delta} \leq \varepsilon / 4$.

On the other hand, there holds that $W(x, 0) \leq 0$ in $\mathbb{R}^{N}$ and $W(x, t) \leq 0$ for $|x| \geq R$ and $t \in[0, T]$. We claim that $W \leq 0$ in $\mathbb{R}^{N} \times[0, T]$. If not, there would exist $\left(x_{0}, t_{0}\right) \in B_{R}(0) \times(0, T]$ a positive maximum for $W$ in the whole region $\mathbb{R}^{N} \times[0, T]$. But this implies

$$
0 \geq \mathcal{L}_{\delta} W\left(x_{0}, t_{0}\right) \geq \varepsilon / 2,
$$

a contradiction and thus, $W \leq 0$ in $\mathbb{R}^{N} \times[0, T]$. Now letting $R \rightarrow \infty$ first and then $\varepsilon \rightarrow 0$ we deduce that $U \leq 0$ in $\mathbb{R}^{N} \times[0, T]$, completing the proof of Step I.

Step II. Now let $u$ and $v$ be as in the statement and, for $P>0$, define $U=e^{-P t}(u-v)$. Then,

$$
\theta \Delta U+(1-\theta)(J * U-U)-U_{t} \geq e^{-P t}(h(u)-h(v))+P U=\tilde{c}(x, t) U+P U=-c(x, t) U,
$$

with $\tilde{c} \in L^{\infty}\left(\mathbb{R}^{N} \times(0, T)\right)$ and $c(x, t)=-\tilde{c}(x, t)-P<0$, if $P$ is taken large enough. It follows that $U$ satisfies (2.1) and therefore $U \leq 0$. The result follows.

Proposition 2.3. Let $u^{\varepsilon}, v^{\varepsilon} \in C\left(\mathbb{R}^{N} \times[0, T]\right) \cap L^{\infty}\left(\mathbb{R}^{N} \times(0, T)\right)$, be such that

$$
\begin{aligned}
\left\{\begin{aligned}
\theta \Delta u^{\varepsilon}+(1-\theta)\left(J * u^{\varepsilon}-u^{\varepsilon}\right)-u_{t}^{\varepsilon} & =\beta_{\varepsilon}\left(u^{\varepsilon}\right)+g^{\varepsilon} & & \text { in } \mathbb{R}^{N} \times(0, T) \\
u^{\varepsilon}(x, 0) & =u_{0}^{\varepsilon}(x) & & \text { in } \mathbb{R}^{N},
\end{aligned}\right. \\
\left\{\begin{aligned}
\theta \Delta v^{\varepsilon}+(1-\theta)\left(J * v^{\varepsilon}-v^{\varepsilon}\right)-v_{t}^{\varepsilon} & =\beta_{\varepsilon}\left(v^{\varepsilon}\right)+f^{\varepsilon} & & \text { in } \mathbb{R}^{N} \times(0, T) \\
v^{\varepsilon}(x, 0) & =v_{0}^{\varepsilon}(x) & & \text { in } \mathbb{R}^{N},
\end{aligned}\right.
\end{aligned}
$$

with $g^{\varepsilon}, f^{\varepsilon} \in L^{\infty}\left(\mathbb{R}^{N} \times(0, T)\right)$ and let $\beta_{\varepsilon}$ be as in Section 1. Assume that $u_{0}^{\varepsilon} \leq v_{0}^{\varepsilon}$ in $\mathbb{R}^{N}$ and $g^{\varepsilon} \geq f^{\varepsilon}$ in $L^{\infty}\left(\mathbb{R}^{N} \times(0, T)\right)$. Then $u^{\varepsilon} \leq v^{\varepsilon}$ in $\mathbb{R}^{N} \times[0, T]$.

Proof. Let $u_{0 \delta}^{\varepsilon}, v_{0 \delta}^{\varepsilon} \in C^{\infty}\left(\mathbb{R}^{N}\right)$ be such that $u_{0 \delta}^{\varepsilon} \leq v_{0 \delta}^{\varepsilon}$ in $\mathbb{R}^{N}, u_{0 \delta}^{\varepsilon} \rightarrow u_{0}^{\varepsilon}, v_{0 \delta}^{\varepsilon} \rightarrow v_{0}^{\varepsilon}$ uniformly on compacts of $\mathbb{R}^{N}$ as $(\delta \rightarrow 0)$

$$
\left\|u_{0 \delta}^{\varepsilon}\right\|_{L^{\infty}\left(\mathbb{R}^{N}\right)} \leq\left\|u_{0}^{\varepsilon}\right\|_{L^{\infty}\left(\mathbb{R}^{N}\right)}, \quad\left\|v_{0 \delta}^{\varepsilon}\right\|_{L^{\infty}\left(\mathbb{R}^{N}\right)} \leq\left\|v_{0}^{\varepsilon}\right\|_{L^{\infty}\left(\mathbb{R}^{N}\right)},
$$

and let $g_{\delta}^{\varepsilon}, f_{\delta}^{\varepsilon} \in C^{\infty}\left(\mathbb{R}^{N} \times[0, T]\right)$ be such that $g_{\delta}^{\varepsilon} \geq f_{\delta}^{\varepsilon}$ in $\mathbb{R}^{N} \times[0, T], g_{\delta}^{\varepsilon} \rightarrow g^{\varepsilon}, f_{\delta}^{\varepsilon} \rightarrow f^{\varepsilon}$ a. e. in $\mathbb{R}^{N} \times(0, T) \quad(\delta \rightarrow 0)$,

$$
\left\|g_{\delta}^{\varepsilon}\right\|_{L^{\infty}\left(\mathbb{R}^{N} \times(0, T)\right)} \leq\left\|g^{\varepsilon}\right\|_{L^{\infty}\left(\mathbb{R}^{N} \times(0, T)\right)}, \quad\left\|f_{\delta}^{\varepsilon}\right\|_{L^{\infty}\left(\mathbb{R}^{N} \times(0, T)\right)} \leq\left\|f^{\varepsilon}\right\|_{L^{\infty}\left(\mathbb{R}^{N} \times(0, T)\right)} .
$$


Now consider $u_{\delta}^{\varepsilon}, v_{\delta}^{\varepsilon} \in C\left(\mathbb{R}^{N} \times\left[0, T_{0}\right]\right) \cap L^{\infty}\left(\mathbb{R}^{N} \times\left(0, T_{0}\right)\right)$, the unique solutions to (2.2) and (2.3) with $T, g^{\varepsilon}, u_{0}^{\varepsilon}, f^{\varepsilon}$ and $v_{0}^{\varepsilon}$ replaced by $T_{0}, g_{\delta}^{\varepsilon}, u_{0 \delta}^{\varepsilon}, f_{\delta}^{\varepsilon}$ and $v_{0 \delta}^{\varepsilon}$ respectively, which exist by Prop. 2.1 for some $T_{0}>0$ independent of $\delta$. Also, from the proof of Prop. 2.1 we deduce that

$$
\left\|u_{\delta}^{\varepsilon}\right\|_{L^{\infty}\left(\mathbb{R}^{N} \times\left(0, T_{0}\right)\right)} \leq 2|| u_{0}^{\varepsilon}\left\|_{L^{\infty}\left(\mathbb{R}^{N}\right)}, \quad\right\| v_{\delta}^{\varepsilon}\left\|_{L^{\infty}\left(\mathbb{R}^{N} \times\left(0, T_{0}\right)\right)} \leq 2\right\| v_{0}^{\varepsilon} \|_{L^{\infty}\left(\mathbb{R}^{N}\right)} .
$$

Standard regularity results give $u_{\delta}^{\varepsilon}, v_{\delta}^{\varepsilon} \in C^{2,1}\left(\mathbb{R}^{N} \times\left(0, T_{0}\right]\right)$ and thus from Prop. 2.2 we get that $u_{\delta}^{\varepsilon} \leq v_{\delta}^{\varepsilon}$ in $\mathbb{R}^{N} \times\left[0, T_{0}\right]$. Then, letting $\delta \rightarrow 0$, we obtain that $u^{\varepsilon} \leq v^{\varepsilon}$ in $\mathbb{R}^{N} \times\left[0, T_{0}\right]$ (here we have used that Prop. 2.1 gives uniqueness to (2.2) and (2.3) up to time $T_{0}$ ).

Let us now define

$$
T^{*}=\sup \left\{0 \leq s \leq T / u^{\varepsilon} \leq v^{\varepsilon} \text { in } \mathbb{R}^{N} \times[0, s]\right\} .
$$

We have $T_{0} \leq T^{*} \leq T$. If $T^{*}<T$ we can repeat the argument above, starting at $t=T^{*}$, and conclude that $u^{\varepsilon} \leq v^{\varepsilon}$ in $\mathbb{R}^{N} \times\left[0, T^{*}+\sigma\right]$, for some $\sigma>0$, which gives a contradiction. Therefore $u^{\varepsilon} \leq v^{\varepsilon}$ in $\mathbb{R}^{N} \times[0, T]$.

Theorem 2.1. Let $u_{0}^{\varepsilon} \in C\left(\mathbb{R}^{N}\right) \cap L^{\infty}\left(\mathbb{R}^{N}\right), g^{\varepsilon} \in L^{\infty}\left(\mathbb{R}^{N} \times(0, T)\right)$ and let $\beta_{\varepsilon}$ be as in Section 1. There exists a unique $u^{\varepsilon} \in C\left(\mathbb{R}^{N} \times[0, T]\right) \cap L^{\infty}\left(\mathbb{R}^{N} \times(0, T)\right)$, solution to

$$
\left\{\begin{array}{rlrl}
\theta \Delta u^{\varepsilon}+(1-\theta)\left(J * u^{\varepsilon}-u^{\varepsilon}\right)-u_{t}^{\varepsilon} & =\beta_{\varepsilon}\left(u^{\varepsilon}\right)+g^{\varepsilon} & & \text { in } \mathbb{R}^{N} \times(0, T) \\
u^{\varepsilon}(x, 0) & =u_{0}^{\varepsilon}(x) & \text { in } \mathbb{R}^{N} .
\end{array}\right.
$$

There holds that $u^{\varepsilon} \in C^{1+\alpha, \frac{1+\alpha}{2}}\left(\mathbb{R}^{N} \times(0, T]\right)$ and

$$
\left\|u^{\varepsilon}\right\|_{L^{\infty}\left(\mathbb{R}^{N} \times(0, T)\right)} \leq T\left\|g^{\varepsilon}\right\|_{L^{\infty}\left(\mathbb{R}^{N} \times(0, T)\right)}+\left\|u_{0}^{\varepsilon}\right\|_{L^{\infty}\left(\mathbb{R}^{N}\right)} .
$$

Thus, if $\left\|u_{0}^{\varepsilon}\right\|_{L^{\infty}\left(\mathbb{R}^{N}\right)} \leq \mathcal{A}_{1}$ and $\left\|g^{\varepsilon}\right\|_{L^{\infty}\left(\mathbb{R}^{N} \times(0, T)\right)} \leq \mathcal{A}_{2}$ for some $\mathcal{A}_{1}>0, \mathcal{A}_{2}>0$, it follows that $\left\|u^{\varepsilon}\right\|_{L^{\infty}\left(\mathbb{R}^{N} \times(0, T)\right)} \leq C$, with $C=C\left(T, \mathcal{A}_{1}, \mathcal{A}_{2}\right)$.

If, in addition, $u_{0}^{\varepsilon} \geq 0$ in $\mathbb{R}^{N}$ and $g^{\varepsilon} \leq 0$ in $\mathbb{R}^{N} \times(0, T)$, then $u^{\varepsilon} \geq 0$ in $\mathbb{R}^{N} \times[0, T]$.

Proof. First assume that such a $u^{\varepsilon}$ exists. Then, uniqueness follows from Prop. 2.3 and standard estimates give that $u^{\varepsilon} \in C^{1+\alpha, \frac{1+\alpha}{2}}\left(\mathbb{R}^{N} \times(0, T]\right)$.

On the other hand, if we let $L=\left\|g^{\varepsilon}\right\|_{L^{\infty}\left(\mathbb{R}^{N} \times(0, T)\right)}, A=\left\|u_{0}^{\varepsilon}\right\|_{L^{\infty}\left(\mathbb{R}^{N}\right)}, w^{\varepsilon}=-t L-A, v^{\varepsilon}=$ $t L+A$, we observe that $w^{\varepsilon}$ and $v^{\varepsilon}$ satisfy

$$
\begin{gathered}
\left\{\begin{array}{rr}
\theta \Delta w^{\varepsilon}+(1-\theta)\left(J * w^{\varepsilon}-w^{\varepsilon}\right)-w_{t}^{\varepsilon}=\beta_{\varepsilon}\left(w^{\varepsilon}\right)+L & \text { in } \mathbb{R}^{N} \times(0, T) \\
w^{\varepsilon}(x, 0)=-A & \text { in } \mathbb{R}^{N},
\end{array}\right. \\
\left\{\begin{array}{rr}
\theta \Delta v^{\varepsilon}+(1-\theta)\left(J * v^{\varepsilon}-v^{\varepsilon}\right)-v_{t}^{\varepsilon}=\beta_{\varepsilon}\left(v^{\varepsilon}\right)+\left(-L-\beta_{\varepsilon}\left(v^{\varepsilon}\right)\right) & \text { in } \mathbb{R}^{N} \times(0, T) \\
v^{\varepsilon}(x, 0)=A & \text { in } \mathbb{R}^{N} .
\end{array}\right.
\end{gathered}
$$

Since $-L-\beta_{\varepsilon}\left(v^{\varepsilon}\right) \leq g^{\varepsilon} \leq L$ and $-A \leq u_{0}^{\varepsilon} \leq A$, we deduce from Prop. 2.3 that

$$
-t L-A \leq u^{\varepsilon} \leq t L+A \text { in } \mathbb{R}^{N} \times[0, T],
$$

and therefore estimate (2.5) follows.

Also the application of Prop. 2.3 will give $u^{\varepsilon} \geq 0$ in $\mathbb{R}^{N} \times[0, T]$, in case there holds that $u_{0}^{\varepsilon} \geq 0$ in $\mathbb{R}^{N}$ and $g^{\varepsilon} \leq 0$ in $\mathbb{R}^{N} \times[0, T]$.

In order to prove existence of such a solution we first notice that Prop. 2.1 gives existence of a solution $u^{\varepsilon} \in C\left(\mathbb{R}^{N} \times\left[0, T_{0}\right]\right) \cap L^{\infty}\left(\mathbb{R}^{N} \times\left(0, T_{0}\right)\right)$ for some $T_{0}>0$. We next deduce from (2.5) that whenever a solution $u^{\varepsilon}$ to $(2.4)$ exists in $C\left(\mathbb{R}^{N} \times[0, s]\right) \cap L^{\infty}\left(\mathbb{R}^{N} \times(0, s)\right)$ with $0<s \leq T$, there holds

$$
\left\|u^{\varepsilon}\right\|_{L^{\infty}\left(\mathbb{R}^{N} \times(0, s)\right)} \leq T\left\|g^{\varepsilon}\right\|_{L^{\infty}\left(\mathbb{R}^{N} \times(0, T)\right)}+\left\|u_{0}^{\varepsilon}\right\|_{L^{\infty}\left(\mathbb{R}^{N}\right)},
$$


and then Prop. 2.1 gives existence of a solution up to time $t=s+\sigma$, with $\sigma>0$ independent of $s$. This implies the existence of a solution $u^{\varepsilon} \in C\left(\mathbb{R}^{N} \times[0, T]\right) \cap L^{\infty}\left(\mathbb{R}^{N} \times(0, T)\right)$ and the theorem follows.

\section{First examples}

The purpose of this section - before continuing with the analysis of the nonlocal problem (1.8) - is to present some examples that will provide important information on the behavior of general solutions of our problem. We also exhibit new examples for the well studied problem $(1.2)$.

Let us first notice that, from the estimates in the preceding section, we know that if $u^{\varepsilon}$ is a family of solutions to

$$
\begin{aligned}
\theta \Delta u^{\varepsilon}+(1-\theta)\left(J * u^{\varepsilon}-u^{\varepsilon}\right)-u_{t}^{\varepsilon} & =\beta_{\varepsilon}\left(u^{\varepsilon}\right)+g^{\varepsilon} \quad \text { in } \mathbb{R}^{N} \times(0, T) \\
u^{\varepsilon}(x, 0) & =u_{0}^{\varepsilon}(x) \quad \text { in } \mathbb{R}^{N},
\end{aligned}
$$

which is under the assumptions of Theorem 2.1, then $\left\|u^{\varepsilon}\right\|_{L^{\infty}\left(\mathbb{R}^{N} \times(0, T)\right)} \leq C$, with $C$ independent of $\varepsilon$. Then, defining

$$
\bar{u}^{\varepsilon}(x, t)=u^{\varepsilon}(\sqrt{\theta} x, t)
$$

it follows that

$$
\Delta \bar{u}^{\varepsilon}-\bar{u}_{t}^{\varepsilon}=\beta_{\varepsilon}\left(\bar{u}^{\varepsilon}\right)+f^{\varepsilon},
$$

with $\left\|\bar{u}^{\varepsilon}\right\|_{L^{\infty}\left(\mathbb{R}^{N} \times(0, T)\right)} \leq C$ and $\left\|f^{\varepsilon}\right\|_{L^{\infty}\left(\mathbb{R}^{N} \times(0, T)\right)} \leq C$, where we have called

$$
f^{\varepsilon}(x, t)=-(1-\theta)\left(J * u^{\varepsilon}-u^{\varepsilon}\right)(\sqrt{\theta} x, t)+g^{\varepsilon}(\sqrt{\theta} x, t) .
$$

On the other hand, proceeding as in the introduction, we can show that solutions to (3.2) can be seen as solutions to the nonlocal equation in (3.1) with a particular forcing term.

With this in mind, we discuss in this section the behavior of the simplest solutions to problem (3.2), i.e, of one dimensional stationary solutions with constant (or piecewise constant) forcing term.

We first discuss the problem without forcing term:

Proposition 3.1. Let $u^{\varepsilon}=u^{\varepsilon}(s)$ be the solution to

$$
\begin{aligned}
u_{s s}^{\varepsilon} & =\beta_{\varepsilon}\left(u^{\varepsilon}\right) \quad s \in \mathbb{R}, \\
u^{\varepsilon}(0) & =\varepsilon, \quad u_{s}^{\varepsilon}(0)=\alpha .
\end{aligned}
$$

Let us denote $u(s)=\lim _{\varepsilon \rightarrow 0} u^{\varepsilon}(s)$. Then,

1) If $\alpha=0$, then $u^{\varepsilon} \equiv \varepsilon$ and $u \equiv 0$.

2) If $0<\alpha<\sqrt{2 M}$, then $\lim _{|s| \rightarrow \infty} u^{\varepsilon}(s)=+\infty$ and $u(s)=\alpha s^{+}+\alpha s^{-}$.

3) If $\alpha=\sqrt{2 M}$, then $u_{s}^{\varepsilon}(s)>0, \lim _{s \rightarrow-\infty} u^{\varepsilon}(s)=0$ and $u(s)=\sqrt{2 M} s^{+}$.

4) If $\alpha>\sqrt{2 M}$, then both $u^{\varepsilon}$ and $u$ change sign.

Proof. When $s>0$ the results follow by direct integration, since $\beta_{\varepsilon} \geq 0$ and $\beta_{\varepsilon}(\tau) \equiv 0$ for $\tau \geq \varepsilon$.

When $s<0$ the results follow from the analysis in Lemma 4.1 in [14], letting there $\psi(s)=$ $\frac{1}{\varepsilon} u^{\varepsilon}(-\varepsilon s)$.

Complementing the previous proposition we obtain: 
Proposition 3.2. Let us assume that there exist $\sigma, \rho_{0}>0$ such that $\beta(\rho) \geq \sigma \rho$ for $\rho \in\left[0, \rho_{0}\right]$. Let $u^{\varepsilon}=u^{\varepsilon}(s)$ be the solution to

$$
\begin{aligned}
u_{s s}^{\varepsilon} & =\beta_{\varepsilon}\left(u^{\varepsilon}\right) \quad s \in \mathbb{R}, \\
u^{\varepsilon}(0) & =\varepsilon, \quad u_{s}^{\varepsilon}(0)=\alpha_{\varepsilon} .
\end{aligned}
$$

Let us denote $u(s)=\lim _{\varepsilon \rightarrow 0} u^{\varepsilon}(s)$ and $B(s)=\int_{0}^{s} \beta(\tau) d \tau$.

Assume that $0<\alpha_{\varepsilon}<\sqrt{2 M}$ with $\alpha_{\varepsilon} \rightarrow \sqrt{2 M}$, as $\varepsilon \rightarrow 0$, and let $a_{\varepsilon}:=B^{-1}\left(M-\frac{1}{2} \alpha_{\varepsilon}^{2}\right)$. Then,

1) If $\left|\varepsilon \ln a_{\varepsilon}\right| \rightarrow 0$, then $u(s)=\sqrt{2 M} s^{+}+\sqrt{2 M} s^{-}$.

2) If $\left|\varepsilon \ln a_{\varepsilon}\right| \rightarrow+\infty$, then $u(s)=\sqrt{2 M} s^{+}$.

3) If $c_{1}<\left|\varepsilon \ln a_{\varepsilon}\right|<c_{2}$ with $0<c_{1}, c_{2}<+\infty$ then, for a subsequence and some $s_{0}<0$, $u(s)=\sqrt{2 M} s^{+}+\sqrt{2 M}\left(s-s_{0}\right)^{-}$.

Proof. Since $0<\alpha_{\varepsilon}<\sqrt{2 M}$, we know from Proposition 3.1 that there exists $r_{\varepsilon}<0$ such that $u_{s}^{\varepsilon}\left(r_{\varepsilon}\right)=0$ and $u_{s}^{\varepsilon}(s)>0$ in $\left(r_{\varepsilon}, 0\right]$.

Let us multiply the equation in (3.4) by $u_{s}^{\varepsilon}$ and then integrate. We get

$$
\frac{1}{2}\left(u_{s}^{\varepsilon}(s)\right)^{2}-\frac{1}{2} \alpha_{\varepsilon}^{2}=B_{\varepsilon}\left(u^{\varepsilon}(s)\right)-M, \text { for } s \in \mathbb{R},
$$

where $B_{\varepsilon}(s)=\int_{0}^{s} \beta_{\varepsilon}(\tau) d \tau$. This implies that

$$
\int_{r_{\varepsilon}}^{0} \frac{u_{s}^{\varepsilon}(s)}{\sqrt{2 B_{\varepsilon}\left(u^{\varepsilon}(s)\right)-2 M+\alpha_{\varepsilon}^{2}}} d s=\int_{r_{\varepsilon}}^{0} d s=\left|r_{\varepsilon}\right| .
$$

On the other hand, the evaluation of (3.5) in $s=r_{\varepsilon}$ implies that $M-\frac{1}{2} \alpha_{\varepsilon}^{2}=B_{\varepsilon}\left(u^{\varepsilon}\left(r_{\varepsilon}\right)\right)$ and thus

$$
\frac{u^{\varepsilon}\left(r_{\varepsilon}\right)}{\varepsilon}=a_{\varepsilon} .
$$

Now changing coordinates in (3.6) and using (3.7) we obtain

$$
\left|r_{\varepsilon}\right|=\frac{\varepsilon}{\sqrt{2}} \int_{a_{\varepsilon}}^{1} \frac{d \rho}{\sqrt{B(\rho)-B\left(a_{\varepsilon}\right)}} .
$$

In order to bound $\left|r_{\varepsilon}\right|$ we first observe that

$$
\frac{\varepsilon}{\sqrt{2}} \int_{\rho_{0}}^{1} \frac{d \rho}{\sqrt{B(\rho)-B\left(a_{\varepsilon}\right)}} \leq \frac{\varepsilon\left(1-\rho_{0}\right)}{\sqrt{2} \sqrt{B\left(\rho_{0}\right)-B\left(a_{\varepsilon}\right)}} .
$$

On the other hand, we have

$$
\frac{\sigma}{2}\left(\rho^{2}-a_{\varepsilon}{ }^{2}\right) \leq B(\rho)-B\left(a_{\varepsilon}\right) \leq \frac{L}{2}\left(\rho^{2}-a_{\varepsilon}{ }^{2}\right) \quad \text { for } a_{\varepsilon} \leq \rho \leq \rho_{0},
$$

where $L$ is the Lipschitz constant of the function $\beta$. This implies that

$$
\frac{\varepsilon}{\sqrt{L}} \int_{1}^{\frac{\rho_{0}}{a_{\varepsilon}}} \frac{d \rho}{\sqrt{\rho^{2}-1}} \leq \frac{\varepsilon}{\sqrt{2}} \int_{a_{\varepsilon}}^{\rho_{0}} \frac{d \rho}{\sqrt{B(\rho)-B\left(a_{\varepsilon}\right)}} \leq \frac{\varepsilon}{\sqrt{\sigma}} \int_{1}^{\frac{\rho_{0}}{a_{\varepsilon}}} \frac{d \rho}{\sqrt{\rho^{2}-1}} .
$$

It is not hard to see that

$$
\varepsilon \ln \rho_{0}-\varepsilon \ln a_{\varepsilon} \leq \varepsilon \int_{1}^{\frac{\rho_{0}}{a_{\varepsilon}}} \frac{d \rho}{\sqrt{\rho^{2}-1}} \leq \varepsilon \ln \left(2 \rho_{0}\right)-\varepsilon \ln a_{\varepsilon}
$$


and thus, putting together (3.8), (3.9), (3.11) and (3.12) we obtain

$$
\frac{1}{\sqrt{L}}\left|\varepsilon \ln a_{\varepsilon}\right|+O(\varepsilon) \leq\left|r_{\varepsilon}\right| \leq \frac{1}{\sqrt{\sigma}}\left|\varepsilon \ln a_{\varepsilon}\right|+O(\varepsilon)
$$

Finally we observe that, whatever the case, we have

$$
u^{\varepsilon}(s)=\alpha_{\varepsilon} s+\varepsilon \quad \text { for } \quad s \geq 0,
$$

$0 \leq u^{\varepsilon}(s) \leq \varepsilon$ for $r_{\varepsilon} \leq s \leq 0$ and $u^{\varepsilon}$ symmetric with respect to $s=r_{\varepsilon}$. This in conjunction with (3.13) gives the desired result.

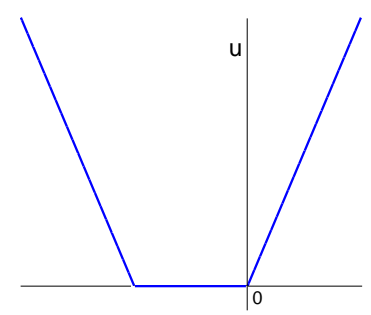

Figure 1. graph of $u$ in Proposition 3.2 when $c_{1}<\left|\varepsilon \ln a_{\varepsilon}\right|<c_{2}$

We next analyze the case of a constant negative forcing term:

Proposition 3.3. Let us assume that there exist $0<a<b<1$ such that $\beta$ is strictly increasing in $(0, a)$ and is strictly decreasing in $(b, 1)$. Let $C>0$ and let $u^{\varepsilon}=u^{\varepsilon}(s)$ be the solution to

$$
\begin{aligned}
u_{s s}^{\varepsilon} & =\beta_{\varepsilon}\left(u^{\varepsilon}\right)-C \quad s \in \mathbb{R}, \\
u^{\varepsilon}(0) & =\varepsilon, \quad u_{s}^{\varepsilon}(0)=\alpha_{\varepsilon} .
\end{aligned}
$$

Let us denote $u(s)=\lim _{\varepsilon \rightarrow 0} u^{\varepsilon}(s)$ and $B_{\varepsilon}(s)=\int_{0}^{s} \beta_{\varepsilon}(\tau) d \tau$. Let $0<s_{\varepsilon}<1$ be the first value such that $\beta\left(s_{\varepsilon}\right)=C \varepsilon$. Then, there exists a critical value

$$
\alpha_{\varepsilon}^{*}=\sqrt{2\left(M-B_{\varepsilon}\left(s_{\varepsilon} \varepsilon\right)+C s_{\varepsilon} \varepsilon-C \varepsilon\right)},
$$

with $\alpha_{\varepsilon}^{*} \rightarrow \sqrt{2 M}$, as $\varepsilon \rightarrow 0$, such that the following situation holds:

1) If $\alpha_{\varepsilon}=0$, then $s_{\varepsilon} \varepsilon<u^{\varepsilon}(s) \leq \varepsilon$ for $s \in \mathbb{R}$ and thus, $u \equiv 0$.

2) If $0<\alpha_{\varepsilon}<\alpha_{\varepsilon}^{*}$, then $u^{\varepsilon}(s)=-\frac{C}{2} s^{2}+\alpha_{\varepsilon} s+\varepsilon$, for $0 \leq s \leq \frac{2 \alpha_{\varepsilon}}{C}$ and there exists $\rho_{\varepsilon}>0$ the smallest value such that $u_{s}^{\varepsilon}\left(-\rho_{\varepsilon}\right)=u_{s}^{\varepsilon}\left(\frac{2 \alpha_{\varepsilon}}{C}+\rho_{\varepsilon}\right)=0$. Thus, $u^{\varepsilon}$ is $\left(\frac{2 \alpha_{\varepsilon}}{C}+2 \rho_{\varepsilon}\right)$-periodic. If, in addition, $\alpha_{\varepsilon} \rightarrow \alpha$ as $\varepsilon \rightarrow 0$ with $0<\alpha<\sqrt{2 M}$, then $\rho_{\varepsilon} \rightarrow 0$ as $\varepsilon \rightarrow 0$,

$$
u \text { is } \frac{2 \alpha}{C} \text {-periodic, } \quad u(s)=\left(-\frac{C}{2} s^{2}+\alpha s\right)^{+} \quad \text { for } 0 \leq s \leq \frac{2 \alpha}{C} \text {. }
$$

3) If $\alpha_{\varepsilon}=\alpha_{\varepsilon}^{*}$, then $u^{\varepsilon}(s)=-\frac{C}{2} s^{2}+\alpha_{\varepsilon} s+\varepsilon$, for $0 \leq s \leq \frac{2 \alpha_{\varepsilon}}{C}, u_{s}^{\varepsilon}(s)>0$ for $s<0, u_{s}^{\varepsilon}(s)<0$ for $s>\frac{2 \alpha_{\varepsilon}}{C}$ and $\lim _{|s| \rightarrow \infty} u^{\varepsilon}(s)=s_{\varepsilon} \varepsilon$. Thus,

$$
u(s)=\left(-\frac{C}{2} s^{2}+\sqrt{2 M} s\right)^{+} .
$$

4) If $\alpha_{\varepsilon}>\alpha_{\varepsilon}^{*}$, then $u^{\varepsilon}$ changes sign. If, in addition, $\alpha_{\varepsilon} \rightarrow \alpha$ as $\varepsilon \rightarrow 0$ with $\alpha>\sqrt{2 M}$, then also $u$ changes sign. 
Proof. Let us first notice that, from our assumptions, it follows that, if $\varepsilon \leq \varepsilon_{0}$, there exist $0<s_{\varepsilon}<\gamma_{\varepsilon}<1$ such that

$$
\beta(s)>C \varepsilon \text { in }\left(s_{\varepsilon}, \gamma_{\varepsilon}\right), \quad \beta(s)<C \varepsilon \text { in }\left(0, s_{\varepsilon}\right) \cup\left(\gamma_{\varepsilon}, 1\right), \quad \beta\left(s_{\varepsilon}\right)=\beta\left(\gamma_{\varepsilon}\right)=C \varepsilon .
$$

Moreover, $\alpha_{\varepsilon}^{*}$ in the statement is well defined, and $\alpha_{\varepsilon}^{*} \rightarrow \sqrt{2 M}$ as $\varepsilon \rightarrow 0$.

Let us now multiply the equation in (3.15) by $u_{s}^{\varepsilon}$ and then integrate. We get

$$
\frac{1}{2}\left(u_{s}^{\varepsilon}(s)\right)^{2}-\frac{1}{2} \alpha_{\varepsilon}^{2}=B_{\varepsilon}\left(u^{\varepsilon}(s)\right)-C u^{\varepsilon}(s)-M+C \varepsilon, \text { for } s \in \mathbb{R},
$$

that can be rewritten as

$$
\frac{1}{2}\left(u_{s}^{\varepsilon}(s)\right)^{2}+\frac{1}{2} \alpha_{\varepsilon}^{* 2}-\frac{1}{2} \alpha_{\varepsilon}^{2}=\int_{s_{\varepsilon} \varepsilon}^{u^{\varepsilon}(s)}\left(\beta_{\varepsilon}(\tau)-C\right) d \tau, \text { for } s \in \mathbb{R} .
$$

I. Assume $\alpha_{\varepsilon}=0$. Then (3.19) implies that $u^{\varepsilon}(s) \leq \varepsilon$ for any $s \in \mathbb{R}$ and from (3.20) we deduce that $s_{\varepsilon} \varepsilon<u^{\varepsilon}(s)$ for any $s \in \mathbb{R}$. Thus, 1) follows.

II. Let us now assume that $0<\alpha_{\varepsilon}<\alpha_{\varepsilon}^{*}$. Then, equation (3.20) implies again that $u^{\varepsilon}(s)>s_{\varepsilon} \varepsilon$ for $s \in \mathbb{R}$.

Also, $u^{\varepsilon}(s)>\varepsilon$ for $s>0$ small and thus $\beta_{\varepsilon}=0$ there. Integrating the equation it follows that

$$
u^{\varepsilon}(s)=-\frac{C}{2} s^{2}+\alpha_{\varepsilon} s+\varepsilon \quad \text { for } \quad 0 \leq s \leq \frac{2 \alpha_{\varepsilon}}{C} .
$$

We claim that there exists $\rho_{\varepsilon}>0$ the smallest value such that $u_{s}^{\varepsilon}\left(-\rho_{\varepsilon}\right)=0$. Otherwise, $u_{s}^{\varepsilon}(s)>0$ for every $s<0$, which implies that there exists $\lim _{s \rightarrow-\infty} u^{\varepsilon}(s)=u_{\infty}^{\varepsilon} \geq s_{\varepsilon} \varepsilon$.

From equation (3.20) we obtain that $u_{\infty}^{\varepsilon}>s_{\varepsilon} \varepsilon$. Now letting $s \rightarrow-\infty$ in the equation in (3.15), we obtain that the only possibility is $u_{\infty}^{\varepsilon}=\gamma_{\varepsilon} \varepsilon$. Hence, $\beta_{\varepsilon}\left(u^{\varepsilon}(s)\right)-C<0$ for every $s<0$, which implies that $u^{\varepsilon}(s) \rightarrow-\infty$, as $s \rightarrow-\infty$, a contradiction.

We have therefore shown that there exists $\rho_{\varepsilon}>0$ the smallest value such that $u_{s}^{\varepsilon}\left(-\rho_{\varepsilon}\right)=0$. Now a reflection argument gives that $u_{s}^{\varepsilon}\left(\frac{2 \alpha_{\varepsilon}}{C}+\rho_{\varepsilon}\right)=0$ and that $u^{\varepsilon}$ is $\left(\frac{2 \alpha_{\varepsilon}}{C}+2 \rho_{\varepsilon}\right)$-periodic.

In order to finish with the proof of 2) let us now assume that $\alpha_{\varepsilon} \rightarrow \alpha$, as $\varepsilon \rightarrow 0$, with $0<\alpha<\sqrt{2 M}$. It only remains to prove that $\rho_{\varepsilon} \rightarrow 0$, as $\varepsilon \rightarrow 0$.

For that purpose we define

$$
v^{\varepsilon}(s)=\frac{1}{\varepsilon} u^{\varepsilon}(\varepsilon s)
$$

and we observe that $v^{\varepsilon} \rightarrow v$ uniformly on compact sets, as $\varepsilon \rightarrow 0$, where $v$ is the solution to

$$
v_{s s}=\beta(v) \quad s \in \mathbb{R}, \quad v(0)=1, \quad v_{s}(0)=\alpha .
$$

From the analysis in Lemma 4.1 in [14] (recall that $0<\alpha<\sqrt{2 M}$ ) we know that there exists $\bar{s}>0$ such that $v(-\bar{s})>2$ and thus $u^{\varepsilon}(-\varepsilon \bar{s})>\varepsilon$, for $\varepsilon$ small. Now using that $u^{\varepsilon}(0)=\varepsilon$ and $u_{s}^{\varepsilon}(0)>0$ we deduce that $-\rho_{\varepsilon} \in(-\varepsilon \bar{s}, 0)$, which implies that $\rho_{\varepsilon} \rightarrow 0$, as $\varepsilon \rightarrow 0$. Therefore 2) follows.

III. Assume that $\alpha_{\varepsilon}=\alpha_{\varepsilon}^{*}$. Then, arguing as in the previous case we get that (3.21) holds.

On the other hand, it is not hard to see that also in this case we have $u^{\varepsilon}>s_{\varepsilon} \varepsilon$. In fact, if there existed $x_{\varepsilon}$ such that $u\left(x_{\varepsilon}\right)=s_{\varepsilon} \varepsilon$, then from (3.20) we would get $u_{s}^{\varepsilon}\left(x_{\varepsilon}\right)=0$ and thus $u^{\varepsilon} \equiv s_{\varepsilon} \varepsilon$ (recall that $\beta\left(s_{\varepsilon} \varepsilon\right)=C \varepsilon$ ) which gives a contradiction.

Let us analyze the behavior of $u^{\varepsilon}$ for $s<0$. We first see that there exists $y_{\varepsilon}>0$ the first value such that $u^{\varepsilon}\left(-y_{\varepsilon}\right)=\gamma_{\varepsilon} \varepsilon$. Otherwise, $\beta_{\varepsilon}\left(u^{\varepsilon}(s)\right)-C<0$ for every $s<0$, implying as in the previous case that $u^{\varepsilon}(s) \rightarrow-\infty$, as $s \rightarrow-\infty$, a contradiction. Also, the fact that $u_{s s}^{\varepsilon}<0$ for $\gamma_{\varepsilon} \varepsilon<u^{\varepsilon}<\varepsilon$ ensures that $u_{s}^{\varepsilon}>0$ for $-y_{\varepsilon} \leq s \leq 0$. 


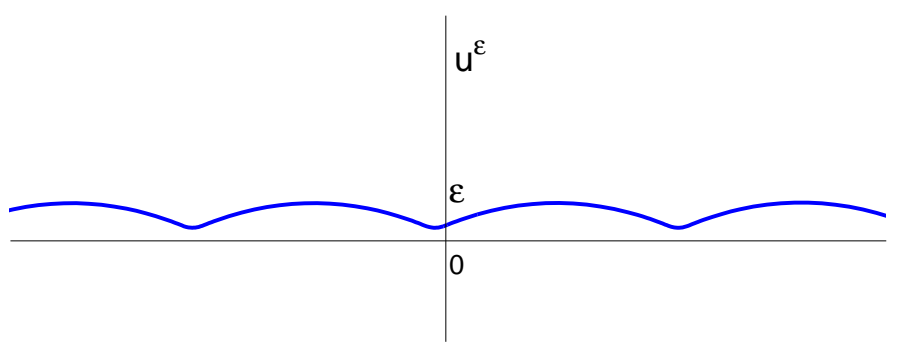

Figure 2. graph of $u^{\varepsilon}$ in Proposition 3.3 for $0<\alpha_{\varepsilon}<\alpha_{\varepsilon}^{*}$

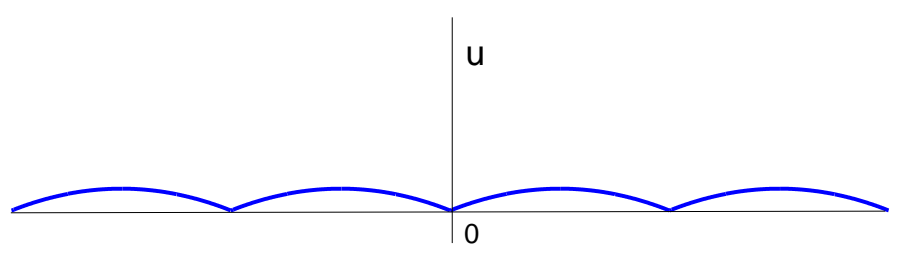

Figure 3. graph of $u$ in Proposition 3.3 when $0<\lim \alpha_{\varepsilon}<\sqrt{2 M}$

We will next show that $u_{s}^{\varepsilon}>0$ also for $s<-y_{\varepsilon}$. We observe that while $s_{\varepsilon} \varepsilon<u^{\varepsilon}(s)<\gamma_{\varepsilon} \varepsilon$ we have $\beta_{\varepsilon}(\tau)-C>0$ in the integrand in (3.20) and thus $u_{s}^{\varepsilon}(s) \neq 0$. Now using that $u_{s}^{\varepsilon}>0$ in a neighborhood of $-y_{\varepsilon}$ we deduce that $s_{\varepsilon} \varepsilon<u^{\varepsilon}<\gamma_{\varepsilon} \varepsilon$ for $s<-y_{\varepsilon}$ and $u_{s}^{\varepsilon}>0$ for $s<0$.

It thus follows that there exists $\lim _{s \rightarrow-\infty} u^{\varepsilon}(s)=u_{\infty}^{\varepsilon}$. The argument above shows that $s_{\varepsilon} \varepsilon \leq u_{\infty}^{\varepsilon}<\gamma_{\varepsilon} \varepsilon$ so letting $s \rightarrow-\infty$ in the equation in (3.15), we obtain that the only possibility is $u_{\infty}^{\varepsilon}=s_{\varepsilon} \varepsilon$.

Finally, a reflection argument gives the behavior of $u^{\varepsilon}$ for $s>\frac{2 \alpha_{\varepsilon}}{C}$, which completes the proof of 3).

IV. Let us now discuss the case $\alpha_{\varepsilon}>\alpha_{\varepsilon}^{*}$. It is enough to study the solutions when $s<0$. Reasoning in a similar way as above, we deduce that it is not possible that $u^{\varepsilon}>\gamma_{\varepsilon} \varepsilon$ for every $s<0$. We also deduce that $u_{s}^{\varepsilon}>0$ while $s_{\varepsilon} \varepsilon \leq u^{\varepsilon} \leq \gamma_{\varepsilon} \varepsilon$.

It follows that $u^{\varepsilon}$ crosses the value $s_{\varepsilon} \varepsilon$ somewhere in $s<0$. But (3.20) also gives that $u_{s}^{\varepsilon}>0$ when $u^{\varepsilon}<s_{\varepsilon} \varepsilon$. This implies that $\lim _{s \rightarrow-\infty} u^{\varepsilon}(s)=-\infty$ and in particular that $u^{\varepsilon}$ changes sign.

It only remains to show that, when $\alpha_{\varepsilon} \rightarrow \alpha$ as $\varepsilon \rightarrow 0$ with $\alpha>\sqrt{2 M}$, also $u$ changes sign. This follows looking again at the functions $v^{\varepsilon}$ in (3.22). In the present case the analysis in Lemma 4.1 in [14] gives that $v=\lim v^{\varepsilon}$ is negative for $s<-\hat{s}<0$. This implies that $u<0$ for $s<0$, completing the proof of 4 ).

Remark 3.1. At the moment of looking for an example of solution to problem (3.2) with positive forcing term, we observe that, since $\beta_{\varepsilon} \geq 0$, a necessary condition for having $\{u \equiv 0\}^{\circ} \neq \emptyset$ for $u=\lim u^{\varepsilon}$, is that $f=\lim f^{\varepsilon}$ be nonpositive on some open set.

Thus, instead of considering the case of a constant positive forcing term, we study: 


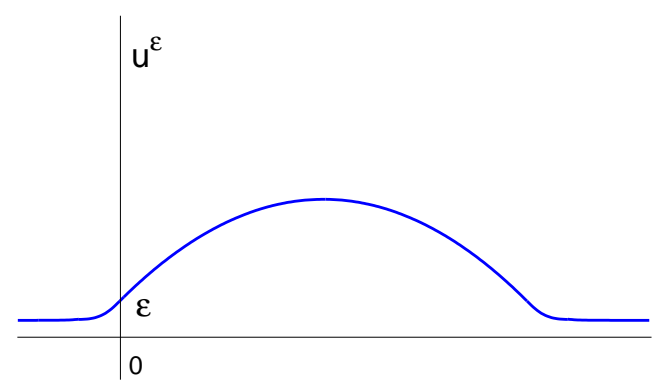

Figure 4. graph of $u^{\varepsilon}$ in Proposition 3.3 for $\alpha_{\varepsilon}=\alpha_{\varepsilon}^{*}$

Proposition 3.4. Let $C>0$ and let $u^{\varepsilon}=u^{\varepsilon}(s)$ be the solution to

$$
\begin{aligned}
u_{s s}^{\varepsilon} & =\beta_{\varepsilon}\left(u^{\varepsilon}\right)+C \chi_{\{s>0\}} \quad s \in \mathbb{R}, \\
u^{\varepsilon}(0) & =\varepsilon, \quad u_{s}^{\varepsilon}(0)=\alpha .
\end{aligned}
$$

Let us denote $u(s)=\lim _{\varepsilon \rightarrow 0} u^{\varepsilon}(s)$. Then,

1) If $\alpha=0$, then $u^{\varepsilon}(s) \equiv \varepsilon$ for $s \leq 0$ and $u^{\varepsilon}(s)=\frac{C}{2} s^{2}+\varepsilon$ for $s \geq 0$. Thus, $u(s)=\frac{C}{2} s^{2} \chi_{\{s>0\}}$.

2) If $0<\alpha<\sqrt{2 M}$, then $\lim _{s \rightarrow-\infty} u^{\varepsilon}(s)=+\infty$ and $u^{\varepsilon}(s)=\frac{C}{2} s^{2}+\alpha s+\varepsilon$ for $s \geq 0$. Thus, $u(s)=\alpha s^{-}+\left(\frac{C}{2} s^{2}+\alpha s\right) \chi_{\{s>0\}}$.

3) If $\alpha=\sqrt{2 M}$, then $u_{s}^{\varepsilon}(s)>0$ for $s<0, \lim _{s \rightarrow-\infty} u^{\varepsilon}(s)=0$ and $u^{\varepsilon}(s)=\frac{C}{2} s^{2}+\sqrt{2 M} s+\varepsilon$ for $s \geq 0$. Thus, $u(s)=\left(\frac{C}{2} s^{2}+\sqrt{2 M} s\right) \chi_{\{s>0\}}$.

4) If $\alpha>\sqrt{2 M}$, then both $u^{\varepsilon}$ and $u$ change sign.

Proof. When $s>0$ the results follow by direct integration, since $\beta_{\varepsilon} \geq 0$ and $\beta_{\varepsilon}(\tau) \equiv 0$ for $\tau \geq \varepsilon$. When $s<0$ the results follow from Proposition 3.1.

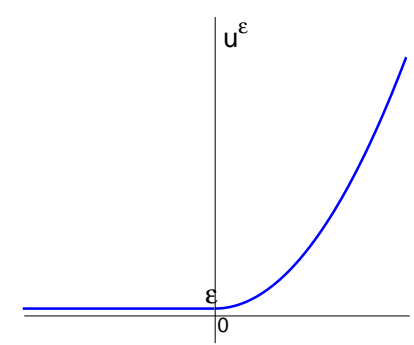

FiguRE 5. graph of $u^{\varepsilon}$ in Proposition 3.4 for $\alpha=0$

Remark 3.2. Notice that, as $C \rightarrow 0$, limit functions in Propositions 3.3 and 3.4 converge to the corresponding limit functions in Proposition 3.1.

\section{Further uniform estimates and passage to the Limit}

Motivated by the analysis presented at the beginning of the previous section, in this section we consider a given family of nonnegative solutions $u^{\varepsilon}(x, t)$ of the equations

$$
\Delta u^{\varepsilon}-u_{t}^{\varepsilon}=\beta_{\varepsilon}\left(u^{\varepsilon}\right)+f^{\varepsilon}
$$


in a domain $\mathcal{D} \subset \mathbb{R}^{N+1}$. We will refer to the equations above as $P_{\varepsilon}\left(f^{\varepsilon}\right)$.

We assume that both families $u^{\varepsilon}$ and $f^{\varepsilon}$ are uniformly bounded in $L^{\infty}$ norm in $\mathcal{D}$, and we obtain further uniform estimates on the family $u^{\varepsilon}$ that allow the passage to the limit, as $\varepsilon \rightarrow 0$.

We also show that the limit function $u$ is a solution of the free boundary problem $P(f)$ :

$$
\begin{array}{ll}
\Delta u-u_{t}=f & \text { in }\{u>0\} \\
|\nabla u|=\sqrt{2 M} & \text { on } \partial\{u>0\}
\end{array}
$$

in a very weak sense. More precisely,

$$
\Delta u-u_{t}-f \chi_{\{u>0\}}=\Lambda
$$

with $\Lambda$ a nonnegative Radon measure supported on the free boundary $\partial\{u>0\}$. Here we have denoted $u=\lim u^{\varepsilon}, f=\lim f^{\varepsilon}$.

We start the section obtaining further uniform estimates for our family:

Theorem 4.1. Let $u^{\varepsilon}$ be a family of nonnegative solutions to $P_{\varepsilon}\left(f^{\varepsilon}\right)$ in a domain $\mathcal{D} \subset \mathbb{R}^{N+1}$ such that $\left\|u^{\varepsilon}\right\|_{L^{\infty}(\mathcal{D})} \leq \mathcal{A}_{1}$ and $\left\|f^{\varepsilon}\right\|_{L^{\infty}(\mathcal{D})} \leq \mathcal{A}_{2}$ for some $\mathcal{A}_{1}>0, \mathcal{A}_{2}>0$. Let $K \subset \mathcal{D}$ be compact and let $\tau>0$ be such that $\mathcal{N}_{2 \tau}^{-}(K) \subset \mathcal{D}$. There exist constants $L=L\left(\tau, \mathcal{A}_{1}, \mathcal{A}_{2}\right)$ and $C=$ $C\left(L, \tau, \mathcal{A}_{1}, \mathcal{A}_{2}\right)$ such that

$$
\left|\nabla u^{\varepsilon}(x, t)\right| \leq L \quad \text { and } \quad\left|u^{\varepsilon}(x, t+\Delta t)-u^{\varepsilon}(x, t)\right| \leq C|\Delta t|^{\frac{1}{2}} \quad \text { for }(x, t),(x, t+\Delta t) \in K .
$$

Proof. The proof follows as in [7] from the next lemma.

Lemma 4.1. Let $u^{\varepsilon}$ be uniformly bounded solutions to $P_{\varepsilon}\left(f^{\varepsilon}\right)$ in $Q_{1}^{-}$with $f^{\varepsilon}$ uniformly bounded. Then, there exists a constant $C$ independent of $\varepsilon$ such that

$$
\left\|\nabla u^{\varepsilon}\right\|_{L^{\infty}\left(Q_{1 / 4}^{-}\right)} \leq C .
$$

Proof. The proof follows as that in [6] for the case $f^{\varepsilon} \equiv 0$. In fact, Lemma 1 in [6] is a general lemma on functions that verify $\left|\Delta u-u_{t}\right| \leq C$. Lemma 2 in [6] does not apply to our one phase case (i.e. $u^{\varepsilon} \geq 0$ ). Lemma 3 in [6] is an energy estimate of the $L^{2}$ norm of $\nabla u^{\varepsilon}$ in $Q_{3 / 4}^{-}$in terms of the $L^{\infty}$ norm of $u^{\varepsilon}$ in $Q_{1}^{-}$in the case that $f^{\varepsilon} \equiv 0$ that also holds for $f^{\varepsilon}$ uniformly bounded in which case the estimate depends also on the norm of $f^{\varepsilon}$. Finally, the actual proof of the result follows in this way: By an approximation argument, we can assume that $u^{\varepsilon}$ and $f^{\varepsilon}$ are smooth. Then, as in [6] we let $\varphi$ be a smooth function, $\varphi \equiv 1$ in $Q_{1 / 2}^{-}$and $\varphi$ decaying linearly to zero when approaching the parabolic boundary of $Q_{2 / 3}^{-}$. Then we let,

$$
M_{1}=\sup _{0 \leq u^{\varepsilon} \leq \varepsilon}\left|\nabla u^{\varepsilon}\right| \varphi \quad M_{2}=\sup _{Q_{1 / 4}^{-}}\left|\nabla u^{\varepsilon}\right|
$$

and prove that $M_{1}$ and $M_{2}$ are bounded independently of $\varepsilon$.

First, one can prove that $M_{1}$ is bounded exactly as in [6]. To this end, let $\left(x_{0}, t_{0}\right) \in Q_{1}^{-}$be such that $u^{\varepsilon}\left(x_{0}, t_{0}\right) \leq \varepsilon$ and $\varphi\left(x_{0}, t_{0}\right)\left|\nabla u^{\varepsilon}\left(x_{0}, t_{0}\right)\right|=M_{1}$ (in particular, $\left.\left(x_{0}, t_{0}\right) \in Q_{2 / 3}^{-}\right)$.

In our case the rescaled functions $\bar{u}^{\varepsilon}(x, t)=\frac{1}{\varepsilon} u^{\varepsilon}\left(x_{0}+\varepsilon x, t_{0}+\varepsilon^{2} t\right)$ are uniformly bounded solutions to

$$
\Delta v-v_{t}=\varepsilon f^{\varepsilon}\left(x_{0}+\varepsilon x, t_{0}+\varepsilon^{2} t\right)+\beta(v) .
$$

Thus, $\left|\Delta \bar{u}^{\varepsilon}-\bar{u}^{\varepsilon} t\right| \leq C$ with $C$ independent of $\varepsilon$. As in [6] we find that there exists $\sigma>0$ independent of $\varepsilon$ such that if $\frac{M_{1}}{\varphi\left(x_{0}, t_{0}\right)}>\frac{1}{\sigma}$ (and this is true if $\left.M_{1}>1 / \sigma\right)$, there exists $(\bar{x}, \bar{t}) \in$ 
$Q_{\frac{\varphi\left(x_{0}, t_{0}\right)}{\sigma M_{1}}}^{-}$such that

$$
\left|\nabla \bar{u}^{\bar{\varepsilon}}(\bar{x}, \bar{t})\right| \geq 2 \frac{M_{1}}{\varphi\left(x_{0}, t_{0}\right)} .
$$

And, rescaling back, there exists $\left(x_{1}, t_{1}\right) \in Q_{\frac{\varepsilon \varphi\left(x_{0}, t_{0}\right)}{\sigma M_{1}}}^{-}\left(x_{0}, t_{0}\right)$ such that

$$
\left|\nabla u^{\varepsilon}\left(x_{1}, t_{1}\right)\right| \geq 2 \frac{M_{1}}{\varphi\left(x_{0}, t_{0}\right)} .
$$

On the other hand, by the definition of $M_{1}$,

$$
\left|\nabla u^{\varepsilon}(x, t)\right| \leq \frac{M_{1}}{\varphi(x, t)} \quad \text { in }\left\{0 \leq u^{\varepsilon} \leq \varepsilon\right\} \cap Q_{1}^{-} .
$$

Since $\varphi$ is Lipschitz continuous there exists $\delta>0$ such that $\varphi(x, t) \geq \frac{2}{3} \varphi\left(x_{0}, t_{0}\right)$ in $Q_{\delta \varphi\left(x_{0}, t_{0}\right)}^{-}\left(x_{0}, t_{0}\right)$. In particular,

$$
\left|\nabla u^{\varepsilon}(x, t)\right| \leq \frac{3}{2} \frac{M_{1}}{\varphi\left(x_{0}, t_{0}\right)} \quad \text { in }\left\{0 \leq u^{\varepsilon} \leq \varepsilon\right\} \cap Q_{\delta \varphi\left(x_{0}, t_{0}\right)}^{-}\left(x_{0}, t_{0}\right) .
$$

Thus, for any direction $e_{i}$, the function $v(x, t)=u_{x_{i}}^{\varepsilon}-\frac{3}{2} \frac{M_{1}}{\varphi\left(x_{0}, t_{0}\right)}$ is a solution to

$$
\Delta v-v_{t}=f_{x_{i}}^{\varepsilon} \quad \text { in }\{v>0\} \cap Q_{\delta \varphi\left(x_{0}, t_{0}\right)}^{-}\left(x_{0}, t_{0}\right) .
$$

Then, (see the proof of Theorem 8.1, Chapter II in [13])

$$
\sup _{Q_{\frac{1}{2} \delta \varphi\left(x_{0}, t_{0}\right)}^{-}\left(x_{0}, t_{0}\right)} v \leq \frac{\tilde{C}}{\delta \varphi\left(x_{0}, t_{0}\right)}\left(1+\|v\|_{L^{2}\left(Q_{\delta \varphi\left(x_{0}, t_{0}\right)}^{-}\left(x_{0}, t_{0}\right)\right)}\right) \leq \frac{C}{\delta \varphi\left(x_{0}, t_{0}\right)}
$$

by the energy estimate. Here $\bar{C}$ depends on the $L^{\infty}$ norm of $f^{\varepsilon}$ and, by the energy estimate, $C$ dependes on the $L^{\infty}$ norms of $f^{\varepsilon}$ and $u^{\varepsilon}$.

Notice that if $M_{1}>\frac{2}{\sigma \delta}$, there holds that $\left(x_{1}, t_{1}\right) \in Q_{\frac{1}{2} \delta \varphi\left(x_{0}, t_{0}\right)}^{-}\left(x_{0}, t_{0}\right)$. If we take $e_{i}$ as the direction of $\nabla u^{\varepsilon}\left(x_{1}, t_{1}\right)$ by applying (4.3) we get

$$
v\left(x_{1}, t_{1}\right)=\left|\nabla u^{\varepsilon}\left(x_{1}, t_{1}\right)\right|-\frac{3}{2} \frac{M_{1}}{\varphi\left(x_{0}, t_{0}\right)} \geq \frac{1}{2} \frac{M_{1}}{\varphi\left(x_{0}, t_{0}\right)} .
$$

This is a contradiction if $M_{1}>\frac{2 C}{\delta}$. Thus, $M_{1}$ is bounded independently of $\varepsilon$.

Now, in order to bound $M_{2}$ we apply again the $L^{\infty}$ estimate in [13] to the function $v=u_{x_{i}}-M_{1}$ which, by the definition of $M_{1}$ and the fact that $\varphi \equiv 1$ in $Q_{1 / 2}^{-}$, is a solution to

$$
\Delta v-v_{t}=f_{x_{i}}^{\varepsilon} \quad \text { in }\{v>0\} \cap Q_{1 / 2}^{-} .
$$

So that, there exists a constant $C$ independent of $\varepsilon$ such that $\sup _{Q_{1 / 4}^{-}} v \leq C$. Therefore, $M_{2}$ is bounded independently of $\varepsilon$ and the result is proved.

With the uniform estimates obtained in Theorem 4.1 we can now pass to the limit as $\varepsilon \rightarrow 0$.

Lemma 4.2. Let $u^{\varepsilon}$ be a family of nonnegative solutions to $P_{\varepsilon}\left(f^{\varepsilon}\right)$ in a domain $\mathcal{D} \subset \mathbb{R}^{N+1}$. Let us assume that $\left\|u^{\varepsilon}\right\|_{L^{\infty}(\mathcal{D})} \leq \mathcal{A}_{1}$ and $\left\|f^{\varepsilon}\right\|_{L^{\infty}(\mathcal{D})} \leq \mathcal{A}_{2}$ for some $\mathcal{A}_{1}>0, \mathcal{A}_{2}>0$. For every $\varepsilon_{n} \rightarrow 0$ there exist a subsequence $\varepsilon_{n^{\prime}} \rightarrow 0, u \in \operatorname{Lip}_{\mathrm{loc}}(1,1 / 2)$ in $\mathcal{D}$ and $f \in L^{\infty}(\mathcal{D})$, such that i) $u^{\varepsilon_{n^{\prime}}} \rightarrow u$ uniformly on compact subsets of $\mathcal{D}$,

ii) $\nabla u^{\varepsilon_{n^{\prime}}} \rightarrow \nabla u$ in $L_{\mathrm{loc}}^{2}(\mathcal{D})$, 
iii) $\frac{\partial}{\partial t} u^{\varepsilon_{n^{\prime}}} \rightarrow \frac{\partial}{\partial t} u$ weakly in $L_{\mathrm{loc}}^{2}(\mathcal{D})$,

iv) $f^{\varepsilon_{n^{\prime}}} \rightarrow f *$-weakly in $L^{\infty}(\mathcal{D})$,

v) $\Delta u-u_{t} \geq f$ in a distributional sense in $\mathcal{D}$.

vi) $\Delta u-u_{t}=f$ in $\mathcal{D} \cap\{u>0\}$.

vii) for every compact $K \subset \mathcal{D}$ there exists $C_{K}>0$, such that

$$
\left\|\frac{\partial u^{\varepsilon}}{\partial t}\right\|_{L^{2}(K)} \leq C_{K}
$$

for every $\varepsilon>0$.

Proof. The proof follows as that of Lemma 3.1 in [7].

We next show that the limit function $u$ is a solution of the free boundary problem $P(f)$ in a very weak sense

Proposition 4.1. Let $u^{\varepsilon_{j}}$ be a family of nonnegative solutions to $P_{\varepsilon_{j}}\left(f^{\varepsilon_{j}}\right)$ in a domain $\mathcal{D} \subset$ $\mathbb{R}^{N+1}$ such that $u^{\varepsilon_{j}} \rightarrow u$ uniformly on compact subsets of $\mathcal{D}, f^{\varepsilon_{j}} \rightarrow f *$-weakly in $L^{\infty}(\mathcal{D})$ and $\varepsilon_{j} \rightarrow 0$. There exists a Radon measure $\mu$ such that $\beta_{\varepsilon_{j}}\left(u^{\varepsilon_{j}}\right) \rightarrow \mu$ as measures in $\mathcal{D}$ and therefore,

$$
\Delta u-u_{t}=\mu+f \quad \text { in } \mathcal{D},
$$

that is, for every $\phi \in C_{c}^{\infty}(\mathcal{D})$,

$$
\iint_{\mathcal{D}}\left(u \phi_{t}-\nabla u \nabla \phi\right) d x d t=\iint_{\mathcal{D}} \phi d \mu+\iint_{\mathcal{D}} f \phi d x d t
$$

There holds that

$$
\begin{aligned}
& \mu=0 \quad \text { in } \mathcal{D} \cap\{u>0\}, \\
& \mu=-f \quad \text { in } \mathcal{D} \cap\{u \equiv 0\}^{\circ},
\end{aligned}
$$

and thus,

$$
\Delta u-u_{t}-f \chi_{\{u>0\}}=\Lambda \quad \text { in } \mathcal{D},
$$

with $\Lambda$ a nonnegative Radon measure supported on the free boundary $\mathcal{D} \cap \partial\{u>0\}$.

Proof. In order to prove (4.4) we only have to find uniform $L^{1}$ estimates of $\beta_{\varepsilon_{j}}\left(u^{\varepsilon_{j}}\right)$. This can be done as in [7], Proposition 3.1.

(4.5) follows by (vi) in the previous lemma. (4.6) is immediate.

Then, $\Lambda=\mu+f-f \chi_{\{u>0\}}$ is a Radon measure supported on the free boundary. Let us see that $\Lambda \geq 0$. To this end, let $\eta \in C_{c}^{\infty}(\mathcal{D})$ be nonnegative and let $\phi_{k}=\eta h(k u)$ where $h(s)=\max (\min (2-s, 1), 0)$. Then,

$$
\int_{\mathcal{D}} \eta d \Lambda=\int_{\mathcal{D} \cap\{u \equiv 0\}} \eta d \Lambda=\lim _{k \rightarrow \infty} \int_{\mathcal{D} \cap\{u \equiv 0\}} \phi_{k} d \Lambda .
$$

Now,

$$
\begin{aligned}
\int_{\mathcal{D} \cap\{u \equiv 0\}} \phi_{k} d \Lambda & =-\int_{\mathcal{D}} \nabla u \nabla \phi_{k}-\int_{\mathcal{D}} u_{t} \phi_{k}-\int_{\mathcal{D}} f \chi_{\{u>0\}} \phi_{k} \\
& \geq-\int_{\mathcal{D} \cap\{0<u<2 / k\}}|\nabla u \nabla \eta|-\int_{\mathcal{D} \cap\{0<u<2 / k\}}\left|u_{t}\right| \eta-\int_{\mathcal{D} \cap\{0<u<2 / k\}}|f| \eta
\end{aligned}
$$


since $\nabla u$ and $u_{t}$ are locally in $L^{2}$. Thus,

$$
\int_{\mathcal{D}} \eta d \Lambda=\lim _{k \rightarrow \infty} \int_{\mathcal{D} \cap\{u \equiv 0\}} \phi_{k} d \Lambda \geq 0 .
$$

Now we state a result that follows from the convergence result (Lemma 4.2) exactly as Lemma 3.2 in [7]. A result analogous to Lemma 3.3 in [7] also holds when $f^{\varepsilon_{j}} \rightarrow 0$.

Lemma 4.3. Let $u^{\varepsilon_{j}}$ be a family of nonnegative solutions to $P_{\varepsilon_{j}}\left(f^{\varepsilon_{j}}\right)$ in a domain $\mathcal{D} \subset \mathbb{R}^{N+1}$ such that $u^{\varepsilon_{j}} \rightarrow u$ uniformly on compact subsets of $\mathcal{D}, f^{\varepsilon_{j}} \rightarrow f *$-weakly in $L^{\infty}(\mathcal{D})$ and $\varepsilon_{j} \rightarrow 0$. Let $\left(x_{0}, t_{0}\right) \in \mathcal{D} \cap \partial\{u>0\}$ and let $\left(x_{n}, t_{n}\right) \in \mathcal{D} \cap \partial\{u>0\}$ be such that $\left(x_{n}, t_{n}\right) \rightarrow\left(x_{0}, t_{0}\right)$ as $n \rightarrow$ $\infty$. Let $\lambda_{n} \rightarrow 0, u_{\lambda_{n}}(x, t)=\frac{1}{\lambda_{n}} u\left(x_{n}+\lambda_{n} x, t_{n}+\lambda_{n}^{2} t\right)$ and $\left(u^{\varepsilon_{j}}\right)_{\lambda_{n}}(x, t)=\frac{1}{\lambda_{n}} u^{\varepsilon_{j}}\left(x_{n}+\lambda_{n} x, t_{n}+\lambda_{n}^{2} t\right)$. Assume that $u_{\lambda_{n}} \rightarrow U$ as $n \rightarrow \infty$ uniformly on compact sets of $\mathbb{R}^{N+1}$. Then, there exists $j(n) \rightarrow+\infty$ such that for every $j_{n} \geq j(n)$ there holds that $\frac{\varepsilon_{j_{n}}}{\lambda_{n}} \rightarrow 0$ and

1) $\left(u^{\varepsilon_{j_{n}}}\right)_{\lambda_{n}} \rightarrow U$ uniformly on compact sets of $\mathbb{R}^{N+1}$,

2) $\nabla\left(u^{\varepsilon_{j_{n}}}\right)_{\lambda_{n}} \rightarrow \nabla U$ in $L_{\mathrm{loc}}^{2}\left(\mathbb{R}^{N+1}\right)$,

3) $\frac{\partial}{\partial t}\left(u^{\varepsilon_{j_{n}}}\right)_{\lambda_{n}} \rightarrow \frac{\partial}{\partial t} U$ weakly in $L_{\mathrm{loc}}^{2}\left(\mathbb{R}^{N+1}\right)$.

Also, we deduce that

4) $\nabla u_{\lambda_{n}} \rightarrow \nabla U$ in $L_{\text {loc }}^{2}\left(\mathbb{R}^{N+1}\right)$,

5) $\frac{\partial}{\partial t} u_{\lambda_{n}} \rightarrow \frac{\partial}{\partial t} U$ weakly in $L_{\mathrm{loc}}^{2}\left(\mathbb{R}^{N+1}\right)$.

Finally, we conclude this section with some comments on the global version of problem $P_{\varepsilon}\left(f^{\varepsilon}\right)$. Namely, given a family $u^{\varepsilon} \in C\left(\mathbb{R}^{N} \times[0, T]\right) \cap L^{\infty}\left(\mathbb{R}^{N} \times(0, T)\right)$ of nonnegative solutions to

$$
\left\{\begin{aligned}
\Delta u^{\varepsilon}-u_{t}^{\varepsilon} & =\beta_{\varepsilon}\left(u^{\varepsilon}\right)+f^{\varepsilon} \quad \text { in } \mathbb{R}^{N} \times(0, T), \\
u^{\varepsilon}(x, 0) & =u_{0}^{\varepsilon}(x) \quad \text { in } \mathbb{R}^{N},
\end{aligned}\right.
$$

with $f^{\varepsilon} \in L^{\infty}\left(\mathbb{R}^{N} \times(0, T)\right)$, we are interested in the uniform properties and the limit as $\varepsilon \rightarrow 0$.

Remark 4.1. Given $u_{0}^{\varepsilon} \in C\left(\mathbb{R}^{N}\right) \cap L^{\infty}\left(\mathbb{R}^{N}\right)$ and $f^{\varepsilon} \in L^{\infty}\left(\mathbb{R}^{N} \times(0, T)\right)$, the results of Section 2 with $\theta=1$ and $g^{\varepsilon}=f^{\varepsilon}$ show that there exists a unique $u^{\varepsilon} \in C\left(\mathbb{R}^{N} \times[0, T]\right) \cap L^{\infty}\left(\mathbb{R}^{N} \times(0, T)\right)$ solution to (4.7).

As a corollary to the results in Section 2 we also obtain

Corollary 4.1. Let $u^{\varepsilon} \in C\left(\mathbb{R}^{N} \times[0, T]\right) \cap L^{\infty}\left(\mathbb{R}^{N} \times(0, T)\right)$ be a family of solutions to (4.7) with $f^{\varepsilon} \in L^{\infty}\left(\mathbb{R}^{N} \times(0, T)\right)$. Assume, in addition, that $\left\|u_{0}^{\varepsilon}\right\|_{L^{\infty}\left(\mathbb{R}^{N}\right)} \leq \mathcal{A}_{1}$ and $\left\|f^{\varepsilon}\right\|_{L^{\infty}}\left(\mathbb{R}^{N} \times(0, T)\right) \leq$ $\mathcal{A}_{2}$ for some $\mathcal{A}_{1}>0, \mathcal{A}_{2}>0$. Then, we have $\left\|u^{\varepsilon}\right\|_{L^{\infty}\left(\mathbb{R}^{N} \times(0, T)\right)} \leq L$, for some $L=L\left(T, \mathcal{A}_{1}, \mathcal{A}_{2}\right)$.

Remark 4.2. Let $u^{\varepsilon}$ be as in Corollary 4.1. Then, the local results proven previously in this section - as well as those in following ones - apply.

Under the same assumptions, a global version of Proposition 4.1 (in $\mathbb{R}^{N} \times[0, T]$ ) analogous to Proposition 4.1 in [7] also holds.

\section{BASIC LIMITS}

In this section we analyze some particular limits of problems $P_{\varepsilon}\left(f^{\varepsilon}\right)$ that are crucial in the understanding of general limits. We recall that $B_{\varepsilon}(s)=\int_{0}^{s} \beta_{\varepsilon}(\tau) d \tau$. 
Lemma 5.1. Let $u^{\varepsilon_{j}}$ be a family of nonnegative solutions to $P_{\varepsilon_{j}}\left(f^{\varepsilon_{j}}\right)$ in a domain $\mathcal{D} \subset \mathbb{R}^{N+1}$ with $\varepsilon_{j} \rightarrow 0$. Let us assume that $\left\|u^{\varepsilon_{j}}\right\|_{L^{\infty}(\mathcal{D})} \leq \mathcal{A}_{1}$ and $\left\|f^{\varepsilon_{j}}\right\|_{L^{\infty}(\mathcal{D})} \leq \mathcal{A}_{2}$ for some $\mathcal{A}_{1}>0$, $\mathcal{A}_{2}>0$. Then, $\left\{B_{\varepsilon_{j}}\left(u^{\varepsilon_{j}}\right)\right\}$ is precompact in $L^{1}\left(\mathcal{D}^{\prime}\right)$ for every $\mathcal{D}^{\prime} \subset \subset \mathcal{D}$. Let $\chi=\lim _{j \rightarrow \infty} B_{\varepsilon_{j}}\left(u^{\varepsilon_{j}}\right)$ (limit in $L_{\mathrm{loc}}^{1}(\mathcal{D})$ for a subsequence). Then $\chi(x, t)=0$ or $\chi(x, t)=M$ almost everywhere in $\mathcal{D}$.

Proof. The proof follows from [18], Proposition 4.1 and Lemma 4.1, where the result was proved when $f^{\varepsilon_{j}}=0$. But the same proof holds for $f^{\varepsilon_{j}} \neq 0$.

Proposition 5.1. Let $u^{\varepsilon_{j}}$ be nonnegative solutions to $P_{\varepsilon_{j}}\left(f^{\varepsilon_{j}}\right)$ in a domain $\mathcal{D} \subset \mathbb{R}^{N+1}$. Let $\left(x_{0}, t_{0}\right) \in \mathcal{D}$ and suppose $u^{\varepsilon_{j}}$ converge to $u=\alpha\left(x-x_{0}\right)_{1}^{+}$uniformly on compact subsets of $\mathcal{D}$, with $\alpha \in \mathbb{R}, f^{\varepsilon_{j}} \rightarrow 0 *$ - weakly in $L^{\infty}(\mathcal{D})$ and $\varepsilon_{j} \rightarrow 0$. Then

$$
\alpha=0 \quad \text { or } \quad \alpha=\sqrt{2 M} \text {. }
$$

Proof. For simplicity we take $x_{0}=0$. Assume $\alpha>0$. As in Proposition 5.2 in [7], let us multiply equation $P_{\varepsilon_{j}}\left(f^{\varepsilon_{j}}\right)$ by $u_{x_{1}}^{\varepsilon_{j}} \varphi$ where $\varphi \in C_{0}^{\infty}(\mathcal{D})$ and let us integrate by parts. Then,

$$
-\frac{1}{2} \iint\left|\nabla u^{\varepsilon_{j}}\right|^{2} \varphi_{x_{1}}+\iint \nabla u^{\varepsilon_{j}} \nabla \varphi u_{x_{1}}^{\varepsilon_{j}}=\iint B_{\varepsilon_{j}}\left(u^{\varepsilon_{j}}\right) \varphi_{x_{1}}-\iint u_{t}^{\varepsilon_{j}} u_{x_{1}}^{\varepsilon_{j}} \varphi-\iint f^{\varepsilon_{j}} u_{x_{1}}^{\varepsilon_{j}} \varphi .
$$

The main difference with [7] is the presence of the term with $f^{\varepsilon_{j}}$. But this term goes to zero as $j \rightarrow \infty$. The other difference is the treatment of the limit of $B_{\varepsilon_{j}}\left(u^{\varepsilon_{j}}\right)$. In [7] it was proved that $\alpha \leq \sqrt{2 M}$ because it was only used the $*$ - weak convergence of $B_{\varepsilon_{j}}\left(u^{\varepsilon_{j}}\right)$ in $L^{\infty}$. In the present situation the treatment is closer to that in Theorem 3.1, Step IV in [15] (see also Lemma 3.1 in [10]): Since we are assuming that $\alpha>0$, using Lemma 5.1 we see that (for a subsequence) $B_{\varepsilon_{j}}\left(u^{\varepsilon_{j}}\right) \rightarrow M \chi_{\left\{x_{1}>0\right\}}+\bar{M}(x, t) \chi_{\left\{x_{1}<0\right\}}$ locally in $L^{1}$, with $\bar{M}(x, t)=0$ or $\bar{M}(x, t)=M$ almost everywhere. Since $\nabla B_{\varepsilon_{j}}\left(u^{\varepsilon_{j}}\right) \rightarrow 0$ in $L_{\mathrm{loc}}^{1}\left(\mathcal{D} \cap\{u \equiv 0\}^{\circ}\right.$ ) (recall that $\beta_{\varepsilon_{j}}\left(u^{\varepsilon_{j}}\right)+f^{\varepsilon_{j}} \rightarrow 0$ as measures in $\mathcal{D} \cap\{u \equiv 0\}^{\circ}$ and $f^{\varepsilon_{j}} \rightarrow 0$ ) we deduce that $\bar{M}=\bar{M}(t)$. Finally, we treat the other terms as in [7] and [15] and conclude that $\alpha=\sqrt{2 M}$.

Proposition 5.2. Let $u^{\varepsilon_{j}}$ be nonnegative solutions to $P_{\varepsilon_{j}}\left(f^{\varepsilon_{j}}\right)$ in a domain $\mathcal{D} \subset \mathbb{R}^{N+1}$. Let $\left(x_{0}, t_{0}\right) \in \mathcal{D}$ and suppose $u^{\varepsilon_{j}}$ converge to $u=\alpha\left(x-x_{0}\right)_{1}^{+}+\bar{\alpha}\left(x-x_{0}\right)_{1}^{-}$uniformly on compact subsets of $\mathcal{D}$, with $\alpha>0, \bar{\alpha}>0, f^{\varepsilon_{j}} \rightarrow 0$ uniformly on compact subsets of $\mathcal{D}$ and $\varepsilon_{j} \rightarrow 0$. Then

$$
\alpha=\bar{\alpha} \leq \sqrt{2 M}
$$

Proof. For simplicity we take $x_{0}=0$ and remove the subindex $j$. Also, we may assume that $Q_{2} \subset \subset \mathcal{D}$. The proof that $\alpha=\bar{\alpha}$ follows as in [7], Proposition 5.2. In order to see that $\alpha \leq \sqrt{2 M}$ we have to modify the arguments in [7].

We proceed by contradiction. Assume $\alpha>\sqrt{2 M}$ and let $u=\alpha x_{1}^{+}+\alpha x_{1}^{-}$. We prove that $u$ is the limit of a sequence $z^{\varepsilon}$ of functions that are symmetric with respect to $x_{1}=0$ and solutions to an equation very similar to $P_{\varepsilon}\left(f^{\varepsilon}\right)$. In fact, let $F_{\varepsilon}=\left\|f^{\varepsilon}\right\|_{\infty}$ and $b_{\varepsilon}=\sup _{Q_{2}}\left|u^{\varepsilon}-u\right|$. Let $z^{\varepsilon}$ be the solution to

$$
\begin{aligned}
\Delta z^{\varepsilon}-z_{t}^{\varepsilon} & =\beta_{\varepsilon}\left(z^{\varepsilon}\right)+F_{\varepsilon} & & \text { in } Q_{2}, \\
z^{\varepsilon} & =u-b_{\varepsilon} & & \text { on } \partial_{p} Q_{2} .
\end{aligned}
$$

Clearly, $z^{\varepsilon}\left(x_{1}, x^{\prime}, t\right)=z^{\varepsilon}\left(-x_{1}, x^{\prime}, t\right)$ and $z^{\varepsilon}$ satisfies the hypotheses of the first sections since they are uniformly bounded. Thus, there exists a function $z$ such that (for a subsequence that we still call $z^{\varepsilon}$ ), $z^{\varepsilon} \rightarrow z$. We want to show that $z=u$.

First, on $\partial_{p} Q_{2}, z^{\varepsilon}=u-\left\|u^{\varepsilon}-u\right\|_{\infty} \leq u^{\varepsilon}$. On the other hand,

$$
\Delta z^{\varepsilon}-z_{t}^{\varepsilon} \geq \beta_{\varepsilon}\left(z^{\varepsilon}\right)+f^{\varepsilon} \quad \text { in } Q_{2} .
$$


Thus, $z^{\varepsilon} \leq u^{\varepsilon}$. Therefore, $z \leq u$. In order to see that $z \geq u$, let $w^{\varepsilon} \in C^{2}(\mathbb{R})$ be such that

$$
w_{s s}^{\varepsilon}=\beta\left(w^{\varepsilon}\right)+\varepsilon F_{\varepsilon}, \quad w^{\varepsilon}(0)=1, \quad w_{s}^{\varepsilon}(0)=\alpha_{\varepsilon},
$$

where $\alpha_{\varepsilon} \rightarrow \alpha$ is such that $\alpha_{\varepsilon}+2 F_{\varepsilon} \leq \alpha$ and $\frac{1}{2} \alpha_{\varepsilon}^{2}-M-\varepsilon F_{\varepsilon}>0$ (recall that $\alpha>\sqrt{2 M}$ ). Then, $w^{\varepsilon}(s)=1+\alpha_{\varepsilon} s+\frac{1}{2} \varepsilon F_{\varepsilon} s^{2}$ if $s \geq 0$. On the other hand, for every $s \in \mathbb{R}$, (with $\left.B(w)=\int_{0}^{w} \beta(u) d u\right)$,

$$
\frac{1}{2}\left(w_{s}^{\varepsilon}(s)\right)^{2}=B\left(w^{\varepsilon}(s)\right)+\frac{1}{2} \alpha_{\varepsilon}^{2}-M+\varepsilon F_{\varepsilon}\left(w^{\varepsilon}(s)-1\right) .
$$

So, as long as $w^{\varepsilon}(s) \geq 0$,

$$
\frac{1}{2}\left(w_{s}^{\varepsilon}(s)\right)^{2} \geq \frac{1}{2} \alpha_{\varepsilon}^{2}-M-\varepsilon F_{\varepsilon}=\frac{1}{2} \gamma_{\varepsilon}^{2}>0 .
$$

Since $\gamma_{\varepsilon} \rightarrow \gamma$ as $\varepsilon \rightarrow 0$ with $\frac{1}{2} \gamma^{2}=\frac{1}{2} \alpha^{2}-M$, there holds that there exists $\bar{\gamma}>0$ such that $\gamma_{\varepsilon} \geq \bar{\gamma}$ if $\varepsilon \leq \varepsilon_{0}$. Thus, there exists $0>\bar{s}_{\varepsilon}>\bar{s}>-\infty$ such that $w^{\varepsilon}\left(\bar{s}_{\varepsilon}\right)=0$ and $w^{\varepsilon}(s)>0$ if $s>\bar{s}_{\varepsilon}$. On the other hand, there exists $\tilde{s}_{\varepsilon}$ such that $w^{\varepsilon}(s)<0$ if $\tilde{s}_{\varepsilon}<s<\bar{s}_{\varepsilon}$ and $w^{\varepsilon}\left(\tilde{s}_{\varepsilon}\right)=0$. There holds,

$$
w_{s}^{\varepsilon}\left(\bar{s}_{\varepsilon}\right)=\gamma_{\varepsilon}, w^{\varepsilon}\left(\bar{s}_{\varepsilon}\right)=0 \text { and } w_{s s}^{\varepsilon}(s)=\varepsilon F_{\varepsilon} \text { in } \tilde{s}_{\varepsilon}<s<\bar{s}_{\varepsilon} .
$$

Thus,

$$
w^{\varepsilon}(s)=\frac{1}{2} \varepsilon F_{\varepsilon}\left(s-\bar{s}_{\varepsilon}\right)^{2}+\gamma_{\varepsilon}\left(s-\bar{s}_{\varepsilon}\right) .
$$

It follows that $\frac{1}{2} \varepsilon F_{\varepsilon}\left(\tilde{s}_{\varepsilon}-\bar{s}_{\varepsilon}\right)+\gamma_{\varepsilon}=0$, so that $\tilde{s}_{\varepsilon}=\bar{s}_{\varepsilon}-\frac{2 \gamma_{\varepsilon}}{\varepsilon F_{\varepsilon}}$. This is,

$$
w^{\varepsilon}(s)=\frac{1}{2} \varepsilon F_{\varepsilon}\left(s-\bar{s}_{\varepsilon}\right)^{2}+\gamma_{\varepsilon}\left(s-\bar{s}_{\varepsilon}\right) \quad \text { if } \quad \bar{s}_{\varepsilon}-\frac{2 \gamma_{\varepsilon}}{\varepsilon F_{\varepsilon}} \leq s \leq \bar{s}_{\varepsilon} .
$$

Since $w^{\varepsilon}$ is a convex function there holds that $w_{s}^{\varepsilon}(s) \leq w_{s}^{\varepsilon}\left(\frac{2}{\varepsilon}+\bar{s}_{\varepsilon}\right)$ if $s \leq \frac{2}{\varepsilon}+\bar{s}_{\varepsilon}$. But, if $\varepsilon$ is small so that $\frac{2}{\varepsilon}+\bar{s}_{\varepsilon}>0$,

$$
w_{s}^{\varepsilon}\left(\frac{2}{\varepsilon}+\bar{s}_{\varepsilon}\right)=\alpha_{\varepsilon}+\varepsilon F_{\varepsilon}\left(\frac{2}{\varepsilon}+\bar{s}_{\varepsilon}\right) \leq \alpha_{\varepsilon}+2 F_{\varepsilon} \leq \alpha .
$$

Thus, if $\varepsilon$ is small, $w_{s}^{\varepsilon}(s) \leq \alpha$ if $s \leq \frac{2}{\varepsilon}+\bar{s}_{\varepsilon}$. Let now $\tilde{b}_{\varepsilon}=b_{\varepsilon}+4 F_{\varepsilon}$ and

$$
\tilde{w}^{\varepsilon}\left(x_{1}\right)=\varepsilon w^{\varepsilon}\left(\frac{x_{1}}{\varepsilon}-\frac{\tilde{b}_{\varepsilon}}{\varepsilon \gamma_{\varepsilon}}+\bar{s}_{\varepsilon}\right) \text {. }
$$

Then,

$$
\Delta \tilde{w}^{\varepsilon}=\beta_{\varepsilon}\left(\tilde{w}^{\varepsilon}\right)+F_{\varepsilon} \quad \text { and } \quad \tilde{w}^{\varepsilon} \leq u-b_{\varepsilon} \quad \text { in } Q_{2} .
$$

In fact, we claim that $\tilde{w}^{\varepsilon}\left(x_{1}\right) \leq-b_{\varepsilon}$ if $-2 \leq x_{1} \leq 0$. In fact, for $x_{1}$ in that range and $\varepsilon \leq \varepsilon_{0}$ small enough, $\tilde{s}_{\varepsilon} \leq \frac{x_{1}}{\varepsilon}-\frac{\tilde{b}_{\varepsilon}}{\varepsilon \gamma_{\varepsilon}}+\bar{s}_{\varepsilon} \leq \bar{s}_{\varepsilon}$. In order to see that this is true we recall that

$$
\tilde{s}_{\varepsilon}=\bar{s}_{\varepsilon}-\frac{1}{\varepsilon} \frac{2 \gamma_{\varepsilon}}{F_{\varepsilon}}, \quad \frac{x_{1}}{\varepsilon}-\frac{\tilde{b}_{\varepsilon}}{\varepsilon \gamma_{\varepsilon}}+\bar{s}_{\varepsilon} \geq \bar{s}_{\varepsilon}-\frac{1}{\varepsilon}\left(2+\frac{\tilde{b}_{\varepsilon}}{\gamma_{\varepsilon}}\right)
$$

and $2+\frac{\tilde{b}_{\varepsilon}}{\gamma_{\varepsilon}} \rightarrow 2 ; \frac{2 \gamma_{\varepsilon}}{F_{\varepsilon}} \rightarrow+\infty$ as $\varepsilon \rightarrow 0$. Thus, if $\varepsilon \leq \varepsilon_{0}$ and $-2 \leq x_{1} \leq 0$,

$$
\tilde{w}^{\varepsilon}\left(x_{1}\right) \leq \frac{1}{2} F_{\varepsilon}\left(2+\frac{\tilde{b}_{\varepsilon}}{\gamma_{\varepsilon}}\right)^{2}-\tilde{b}_{\varepsilon}=\frac{1}{2} F_{\varepsilon}\left(2+\frac{\tilde{b}_{\varepsilon}}{\gamma_{\varepsilon}}\right)^{2}-4 F_{\varepsilon}-b_{\varepsilon} \leq-b_{\varepsilon},
$$

if $\left(2+\frac{\tilde{b}_{\varepsilon}}{\gamma_{\varepsilon}}\right)^{2} \leq 8$ and this holds if $\varepsilon$ is small enough since $\tilde{b}_{\varepsilon} \rightarrow 0$. Then, since $u \geq 0$ in $\left\{x_{1}<0\right\}$, there holds that $\tilde{w}^{\varepsilon}\left(x_{1}\right) \leq u-b_{\varepsilon}$ if $-2 \leq x_{1} \leq 0$. On the other hand, if $x_{1} \leq 2$ there holds that 
$\frac{x_{1}}{\varepsilon}-\frac{\tilde{b}_{\varepsilon}}{\varepsilon \gamma_{\varepsilon}}+\bar{s}_{\varepsilon} \leq \frac{2}{\varepsilon}+\bar{s}_{\varepsilon}$. So that, $\tilde{w}^{\varepsilon} x_{1}\left(x_{1}\right) \leq \alpha$. This, together with the fact that $\tilde{w}^{\varepsilon}(0) \leq-b_{\varepsilon}$ implies that

$$
\tilde{w}^{\varepsilon}\left(x_{1}\right) \leq \alpha x_{1}-b_{\varepsilon}=u-b_{\varepsilon} \quad \text { if } 0 \leq x_{1} \leq 2 .
$$

So that, since $z^{\varepsilon}=u-b_{\varepsilon}$ on $\partial_{p} Q_{2}$, there holds that $\tilde{w}^{\varepsilon} \leq z^{\varepsilon}$ in $Q_{2}$.

We will see that $\tilde{w}^{\varepsilon} \rightarrow u$ in $Q_{2} \cap\left\{x_{1}>0\right\}$. This will imply that $u \leq z$ in $Q_{2} \cap\left\{x_{1}>0\right\}$. Finally, the fact that $u$ and $z$ are symmetric with respect to $x_{1}=0$ implies that $u \leq z$ in $Q_{2}$.

So that $u=z=\lim z^{\varepsilon}$ as we wanted to prove.

So, let us see that $\tilde{w}^{\varepsilon} \rightarrow u$ in $Q_{2} \cap\left\{x_{1}>0\right\}$. Let $0<x_{1} \leq 2$, then for $\varepsilon$ small enough, as $\tilde{b}_{\varepsilon} \rightarrow 0, x_{1}>\frac{\tilde{b}_{\varepsilon}}{\gamma_{\varepsilon}}-\bar{s}_{\varepsilon} \varepsilon$. Thus,

$$
\tilde{w}^{\varepsilon}\left(x_{1}\right)=\varepsilon+\alpha_{\varepsilon} \varepsilon\left(\frac{x_{1}}{\varepsilon}-\frac{\tilde{b}_{\varepsilon}}{\varepsilon \gamma_{\varepsilon}}+\bar{s}_{\varepsilon}\right)+\frac{1}{2} \varepsilon^{2} F_{\varepsilon}\left(\frac{x_{1}}{\varepsilon}-\frac{\tilde{b}_{\varepsilon}}{\varepsilon \gamma_{\varepsilon}}+\bar{s}_{\varepsilon}\right)^{2} \rightarrow \alpha x_{1} .
$$

In conclusion, assuming that $\alpha>\sqrt{2 M}$ we see that there exist functions $z^{\varepsilon}$ which are symmetric with respect to $x_{1}=0$, solutions to

$$
\Delta z^{\varepsilon}-z_{t}^{\varepsilon}=\beta_{\varepsilon}\left(z^{\varepsilon}\right)+F_{\varepsilon}
$$

with $F_{\varepsilon} \rightarrow 0$, that converge to $u=\alpha x_{1}^{+}+\alpha x_{1}^{-}$as $\varepsilon \rightarrow 0$. Let us see that necessarily $\alpha \leq \sqrt{2 M}$. This will be a contradiction thus proving that $\alpha \leq \sqrt{2 M}$ in the first place.

Multiply (5.1) by $z_{x_{1}}^{\varepsilon}$ and integrate in $\mathcal{R}=\left\{0<x_{1}<1,\left|x^{\prime}\right|<1 .|t|<1\right\}$. We get,

$$
\iint_{\mathcal{R}} z_{x_{1} x_{1}}^{\varepsilon} z_{x_{1}}^{\varepsilon}-\iint_{\mathcal{R}} \beta_{\varepsilon}\left(z^{\varepsilon}\right) z_{x_{1}}^{\varepsilon}=\iint_{\mathcal{R}} z_{t}^{\varepsilon} z_{x_{1}}^{\varepsilon}-\iint_{\mathcal{R}} \Delta_{x^{\prime}} z^{\varepsilon} z_{x_{1}}^{\varepsilon}+\iint_{\mathcal{R}} F_{\varepsilon} z_{x_{1}}^{\varepsilon} .
$$

Let

$$
E_{\varepsilon}:=\iint_{\mathcal{R}} \frac{\partial}{\partial x_{1}}\left(\frac{1}{2}\left(z_{x_{1}}^{\varepsilon}\right)^{2}-B_{\varepsilon}\left(z^{\varepsilon}\right)\right)=\iint_{\mathcal{R}} z_{t}^{\varepsilon} z_{x_{1}}^{\varepsilon}-\iint_{\mathcal{R}} \Delta_{x^{\prime}} z^{\varepsilon} z_{x_{1}}^{\varepsilon}+\iint_{\mathcal{R}} F_{\varepsilon} z_{x_{1}}^{\varepsilon} .
$$

Since $F_{\varepsilon} \rightarrow 0,\left|\nabla z^{\varepsilon}\right| \leq C, z_{t}^{\varepsilon} \rightarrow 0$ in $L^{2}\left(Q_{1}\right)$,

$$
\limsup _{\varepsilon \rightarrow 0} E_{\varepsilon}=-\liminf _{\varepsilon \rightarrow 0} \iint_{\mathcal{R}} \Delta_{x^{\prime}} z^{\varepsilon} z_{x_{1}}^{\varepsilon}
$$

We see that $\liminf \operatorname{in}_{\varepsilon \rightarrow 0} \iint_{\mathcal{R}} \Delta_{x^{\prime}} z^{\varepsilon} z_{x_{1}}^{\varepsilon} \geq 0$. This is done as in [7] since the estimate

$$
\left\|\nabla\left(z^{\varepsilon}-u\right)\right\|_{L^{\infty}\left(Q_{r / 2}^{-}\left(x_{0}, t_{0}\right)\right)} \leq C\left\{\left\|z^{\varepsilon}-u\right\|_{L^{\infty}\left(Q_{r}^{-}\left(x_{0}, t_{0}\right)\right)}+F_{\varepsilon}\right\} \quad \text { if } Q_{r}^{-}\left(x_{0}, t_{0}\right) \subset \subset Q_{2} \backslash\left\{x_{1}=0\right\}
$$

gives the uniform convergence of $\nabla z^{\varepsilon}$ to $\nabla u$ on compact subsets of $Q_{2} \backslash\left\{x_{1}=0\right\}$.

Thus, $\lim _{\sup _{\varepsilon \rightarrow 0}} E_{\varepsilon} \leq 0$. Now the proof follows as in [7].

Remark 5.1. Let $0 \leq \alpha \leq \sqrt{2 M}$. The analysis in Section 3 provides us with examples of families $u^{\varepsilon_{j}}$ of nonnegative solutions to $P_{\varepsilon_{j}}\left(f^{\varepsilon_{j}}\right)$ in $\mathbb{R}^{N+1}$, with $f^{\varepsilon_{j}} \rightarrow 0$ uniformly on compact sets of $\mathbb{R}^{N}$ such that $u^{\varepsilon_{j}} \rightarrow u=\alpha x_{1}^{+}+\alpha x_{1}^{-}$uniformly on compact sets of $\mathbb{R}^{N+1}$.

\section{BeHAVIOR OF LIMIT FUNCTIONS NEAR THE FREE BOUNDARY}

In this section we analyze the behavior of limit functions $u=\lim u^{\varepsilon}$, with $u^{\varepsilon}$ a family of solutions to problems $P_{\varepsilon}\left(f^{\varepsilon}\right)$.

The first result (Theorem 6.1) says that a limit function is, in a sense that we may call pseudo-classical, a supersolution to the free boundary problem $P(f)$ - this holding for any limit function, without imposing any additional hypothesis. 
Then, we prove that the limit function $u$ is nondegenerate at a free boundary point if there is a vertical hyperplane such that the parabolic density of the set $\{u>0\}$ at one side and that of $\{u \equiv 0\}$ at the other side are large enough at the point and $u^{\varepsilon}$ goes to zero fast enough in $\{u \equiv 0\}$ (Theorem 6.2).

Theorem 6.1. Let $u^{\varepsilon_{j}}$ be nonnegative solutions to $P_{\varepsilon_{j}}\left(f^{\varepsilon_{j}}\right)$ in a domain $\mathcal{D} \subset \mathbb{R}^{N+1}$ such that $u^{\varepsilon_{j}} \rightarrow u$ uniformly on compact subsets of $\mathcal{D}, f^{\varepsilon_{j}} \rightarrow f *$-weakly in $L^{\infty}(\mathcal{D})$ and $\varepsilon_{j} \rightarrow 0$. Let $\left(x_{0}, t_{0}\right) \in \mathcal{D} \cap \partial\{u>0\}$. Then,

$$
\limsup _{(x, t) \rightarrow\left(x_{0}, t_{0}\right)}|\nabla u(x, t)| \leq \sqrt{2 M} .
$$

Proof. The proof follows as that of Theorem 6.1 in [7]. In fact, since $\left\|f^{\varepsilon_{j}}\right\|_{\infty} \leq C$, for $d_{n} \rightarrow 0$, $\varepsilon_{n}^{0}:=\frac{\varepsilon_{j_{n}}}{d_{n}} \rightarrow 0,\left(z_{n}, s_{n}\right) \rightarrow\left(x_{0}, t_{0}\right)$, there holds that $f^{\varepsilon_{n}^{0}}(x, t)=d_{n} f^{\varepsilon_{j_{n}}}\left(z_{n}+d_{n} x, s_{n}+d_{n}^{2} t\right)$ converge uniformly to 0 as $n \rightarrow \infty$ on compact sets. Let $u^{\varepsilon_{n}^{0}}(x, t)=\frac{1}{d_{n}} u^{\varepsilon_{j_{n}}}\left(z_{n}+d_{n} x, s_{n}+d_{n}^{2} t\right)$. Then, $u^{\varepsilon_{n}^{0}}$ are solutions to $P_{\varepsilon_{n}^{0}}\left(f^{\varepsilon_{n}^{0}}\right)$. The proof follows as in [7] by applying Propositions 5.1 and 5.2 to rescalings of $u^{\varepsilon_{n}^{0}}$. We are using that any blow up limit $u_{0}$ of $u$ is a solution of the homogenous heat equation in the set $\left\{u_{0}>0\right\}$.

A result similar to Theorem 6.2 in [7], when $U \geq 0$ and $f^{\varepsilon_{j}} \rightarrow 0$, also follows.

Theorem 6.2. Let $u^{\varepsilon_{j}}$ be nonnegative solutions to $P_{\varepsilon_{j}}\left(f^{\varepsilon_{j}}\right)$ in a domain $\mathcal{D} \subset \mathbb{R}^{N+1}$ such that $u^{\varepsilon_{j}} \rightarrow u$ uniformly on compact subsets of $\mathcal{D}, f^{\varepsilon_{j}} \rightarrow f *$-weakly in $L^{\infty}(\mathcal{D})$ and $\varepsilon_{j} \rightarrow 0$. Let $\left(x_{0}, t_{0}\right) \in \mathcal{D} \cap \partial\{u>0\}$. Assume that $\frac{u^{\varepsilon_{j}}}{\varepsilon_{j}} \rightarrow 0$ a.e. in $\mathcal{D} \cap\{u \equiv 0\}^{\circ} \cap\left\{t \leq t_{0}\right\}$ and that there exists $\nu \in \mathbb{R}^{N}$, with $|\nu|=1$ such that

$$
\liminf _{r \rightarrow 0^{+}} \frac{\left|\{u>0\} \cap\left\{\left\langle x-x_{0}, \nu\right\rangle>0\right\} \cap Q_{r}^{-}\left(x_{0}, t_{0}\right)\right|}{\left|Q_{r}^{-}\left(x_{0}, t_{0}\right)\right|}=\alpha_{1},
$$

and

$$
\liminf _{r \rightarrow 0^{+}} \frac{\left|\{u \equiv 0\}^{\circ} \cap\left\{\left\langle x-x_{0}, \nu\right\rangle<0\right\} \cap Q_{r}^{-}\left(x_{0}, t_{0}\right)\right|}{\left|Q_{r}^{-}\left(x_{0}, t_{0}\right)\right|}=\alpha_{2}
$$

with $\alpha_{1}+\alpha_{2}>\frac{1}{2}$. Then, there exists a constant $C>0$ such that, for every $r>0$ small,

$$
\sup _{\partial_{p} Q_{r}^{-}\left(x_{0}, t_{0}\right)} u \geq C r .
$$

The constant $C$ depends only on $\alpha_{1}+\alpha_{2}, N$ and the function $\beta$.

Proof. The proof was done in [7], Theorem 6.3, for the strictly two phase case and $f^{\varepsilon_{j}}=0$. For the one phase case (in a more general setting that consists of a system of equations with unknowns $u^{\varepsilon_{j}}$ and $v^{\varepsilon_{j}}$, that reduces to $P_{\varepsilon_{j}}\left(f^{\varepsilon_{j}}\right)$ when $\left.u^{\varepsilon_{j}}=v^{\varepsilon_{j}}\right)$ and still with $f^{\varepsilon_{j}}=0$ it was proved in [10], Proposition 4.1 and Remark 4.1.

In the present situation we follow the lines of the proof in [10] in order to prove that

$$
\iint_{Q_{1}^{-}} \beta_{\varepsilon_{j} / r}\left(\left(u^{\varepsilon_{j}}\right)_{r}\right) \geq c
$$

for a certain positive constant $c$. Here $\left(u^{\varepsilon_{j}}\right)_{r}(x, t)=\frac{1}{r} u^{\varepsilon_{j}}\left(x_{0}+r x, t_{0}+r^{2} t\right)$. Let us sketch the idea of the proof of (6.3). By (6.1) and (6.2) with $\alpha_{1}+\alpha_{2}>1 / 2$, the fact that $u^{\varepsilon_{j}} / \varepsilon_{j} \rightarrow 0$ a.e. in $\{u \equiv 0\}^{\circ}$ and the uniform Lipschitz continuity of $u^{\varepsilon_{j}}$, we can prove that for every $0<\eta<1$ 
there exists $r_{0}>0$ such that if $0<r \leq r_{0}$ and $0<\varepsilon_{j} \leq \varepsilon_{0}(r)$ there exists $A \subset Q_{1}^{-}$with $|A| \geq c \varepsilon_{j} / r$ such that $\eta \leq \frac{\left(u^{\varepsilon_{j}}\right)_{r}}{\varepsilon_{j} / r} \leq 1-\eta$ in $A$. From this (6.3) follows immediately.

Now the proof follows as in [7]. In fact, $\left(u^{\varepsilon_{j}}\right)_{r}$ are solutions to $P_{\varepsilon_{j}}\left(\bar{f}_{r}^{\varepsilon_{j}}\right)$ where $\bar{f}_{r}^{\varepsilon_{j}}(x, t)=$ $r f^{\varepsilon_{j}}\left(x_{0}+r x, t_{0}+r^{2} t\right) \rightarrow 0$ uniformly on compact sets of $\mathbb{R}^{N+1}$. Let us prove that there exists $C>0$ such that, for $r$ small

$$
\sup _{\partial_{p} Q_{r}^{-}\left(x_{0}, t_{0}\right)} u \geq C r .
$$

If not, there exists $r_{n} \rightarrow 0$ such that $\sup _{\partial_{p} Q_{r_{n}}^{-}\left(x_{0}, t_{0}\right)} u \leq r_{n} / n$. Since $u^{\varepsilon_{j}} \rightarrow u$ uniformly in a neighborhood of $\left(x_{0}, t_{0}\right)$,

$$
\sup _{\partial_{p} Q_{r_{n}}^{-}\left(x_{0}, t_{0}\right)} u^{\varepsilon_{j}} \leq \frac{2}{n} r_{n} \quad \text { for } j \geq j_{0}(n) .
$$

On the other hand, since $\left(u^{\varepsilon_{j}}\right)_{r}$ are solutions to $P_{\varepsilon_{j}}\left(\bar{f}_{r}^{\varepsilon_{j}}\right)$,

$$
\left(u^{\varepsilon_{j}}\right)_{r_{n}}(0,0)=\int_{\partial_{p} Q_{2}^{-}}\left(u^{\varepsilon_{j}}\right)_{r_{n}} P-\iint_{Q_{2}^{-}} \beta_{\varepsilon_{j} / r_{n}}\left(\left(u^{\varepsilon_{j}}\right)_{r_{n}}\right) G-\iint_{Q_{2}^{-}} \bar{f}_{r_{n}}^{\varepsilon_{j}} G
$$

where

$$
P \geq 0 \text { in } \partial_{p} Q_{2}^{-}, \quad \int_{\partial_{p} Q_{2}^{-}} P=1, \quad G \geq 0 \text { in } Q_{2}^{-}, \quad \iint_{Q_{2}^{-}} G \leq K, \quad G \geq \mu>0 \text { in } Q_{1}^{-} .
$$

Thus, by (6.3) and the fact that $\bar{f}_{r}^{\varepsilon_{j}} \rightarrow 0$ uniformly in $Q_{1}^{-}$as $r \rightarrow 0$, there holds that $\left(u^{\varepsilon_{j}}\right)_{r_{n}}(0,0) \leq 2 / n-\mu c+K \delta$, for every $\delta>0$ if $n$ is large enough and $j \geq j_{1}(n)$. So that,

$$
\left(u^{\varepsilon_{j}}\right)_{r_{n}}(0,0) \leq-\frac{\mu}{2} c
$$

if $n$ is large and $j \geq j_{2}(n)$, a contradiction since $\left(u^{\varepsilon_{j}}\right)_{r_{n}}(0,0) \rightarrow 0$ as $j \rightarrow \infty$. The theorem is proved.

\section{Asymptotic Development at Regular fReE Boundary points}

In this section we consider $u=\lim u^{\varepsilon}$, with $u^{\varepsilon}$ solutions to problems $P_{\varepsilon}\left(f^{\varepsilon}\right)$, and we prove that the free boundary condition

$$
|\nabla u|=\sqrt{2 M}
$$

is satisfied in a pointwise sense at any point $\left(x_{0}, t_{0}\right) \in \partial\{u>0\}$ that has an inward unit spatial normal in the parabolic measure theoretic sense (see Definition 7.1). The result holds if $u$ satisfies a nondegeneracy condition at the point (see Definition 7.3).

In Theorem 7.1 we prove the asymptotic development for $t \leq t_{0}$ with assumptions only at the free boundary point. This result is new even when $f^{\varepsilon} \equiv 0$. On the other hand, in Theorem 7.2 we get the asymptotic development also for $t \geq t_{0}$ if $u^{\varepsilon} / \varepsilon$ converges to zero fast enough in $\{u \equiv 0\}$ in a neighborhood of the free boundary point.

We remark that, as shown by the examples in Section 3, an assumption that guarantees nondegeneracy is essential in order to get the free boundary condition (7.1). 
Definition 7.1. We say that $\nu$ is the inward unit spatial normal to the free boundary $\partial\{u>0\}$ at a point $\left(x_{0}, t_{0}\right) \in \partial\{u>0\}$, for $t \leq t_{0}$, in the parabolic measure theoretic sense, if $\nu \in \mathbb{R}^{N}$, $|\nu|=1$ and

$$
\lim _{r \rightarrow 0} \frac{1}{r^{N+2}} \iint_{Q_{r}^{-}\left(x_{0}, t_{0}\right)}\left|\chi_{\{u>0\}}-\chi_{\left\{(x, t) /\left\langle x-x_{0}, \nu\right\rangle>0\right\}}\right| d x d t=0 .
$$

Definition 7.2. We say that a point $\left(x_{0}, t_{0}\right) \in \partial\{u>0\}$ is regular if there exists an inward unit spatial normal to $\partial\{u>0\}$ at $\left(x_{0}, t_{0}\right)$, for $t \leq t_{0}$, in the parabolic measure theoretic sense.

Definition 7.3. We say that $u$ is nondegenerate at $\left(x_{0}, t_{0}\right) \in \partial\{u>0\}$ if there exists a constant $C>0$ such that, for every $r>0$ small,

$$
\sup _{\partial_{p} Q_{r}^{-}\left(x_{0}, t_{0}\right)} u \geq C r .
$$

Theorem 7.1. Let $u^{\varepsilon_{j}}$ be nonnegative solutions to $P_{\varepsilon_{j}}\left(f^{\varepsilon_{j}}\right)$ in a domain $\mathcal{D} \subset \mathbb{R}^{N+1}$ such that $u^{\varepsilon_{j}}$ converge to $u$ uniformly on compact subsets of $\mathcal{D}, f^{\varepsilon_{j}} \rightarrow f$-weakly in $L^{\infty}(\mathcal{D})$ and $\varepsilon_{j} \rightarrow 0$. Let $\left(x_{0}, t_{0}\right) \in \mathcal{D} \cap \partial\{u>0\}$ be a regular point. Assume that $u$ is nondegenerate at $\left(x_{0}, t_{0}\right)$. Then, for $t \leq t_{0}$,

$$
u(x, t)=\sqrt{2 M}\left\langle x-x_{0}, \nu\right\rangle^{+}+o\left(\left|x-x_{0}\right|+\left|t-t_{0}\right|^{\frac{1}{2}}\right)
$$

where $\nu$ is the inward unit spatial normal to $\partial\{u>0\}$ at $\left(x_{0}, t_{0}\right)$, for $t \leq t_{0}$, in the parabolic measure theoretic sense.

Proof. We will assume, without loss of generality, that $\left(x_{0}, t_{0}\right)=(0,0)$ and $\nu=e_{1}$. Let $u_{\lambda}(x, t)=$ $\frac{1}{\lambda} u\left(\lambda x, \lambda^{2} t\right)$, and let $r>0$ be such that $Q_{r}(0,0) \subset \subset \mathcal{D}$. We have that $u_{\lambda} \in \operatorname{Lip}(1,1 / 2)$ in $Q_{r / \lambda}(0,0)$ uniformly in $\lambda$, and $u_{\lambda}(0,0)=0$. Therefore, for every $\lambda_{n} \rightarrow 0$, there exists a subsequence, that we still call $\lambda_{n}$, and a function $U \in \operatorname{Lip}(1,1 / 2)$ in $\mathbb{R}^{N+1}$ such that $u_{\lambda_{n}} \rightarrow U$ uniformly on compact sets of $\mathbb{R}^{N+1}$.

Our aim is to prove that $U=\sqrt{2 M} x_{1}^{+}$for $t \leq 0$.

By (7.2), it follows that for every $k>0$, as $\lambda \rightarrow 0$

$$
\left|\left\{u_{\lambda}>0\right\} \cap\left\{x_{1}<0\right\} \cap Q_{k}^{-}(0,0)\right| \rightarrow 0, \quad\left|\left\{u_{\lambda} \equiv 0\right\} \cap\left\{x_{1}>0\right\} \cap Q_{k}^{-}(0,0)\right| \rightarrow 0 .
$$

This implies that $U \equiv 0$ in $\left\{x_{1}<0\right\} \cap\{t \leq 0\}$. On the other hand, $U$ is nonnegative and a solution of the homogeneous heat equation in $\{U>0\}$, and $\{U>0\} \cap\{t<0\} \subset\left\{x_{1}>0\right\}$. By Corollary A.1 in [7], there exists $\alpha \geq 0$ such that

$$
U(x, t)=\alpha x_{1}^{+}+o\left(|x|+|t|^{\frac{1}{2}}\right) \text { in }\left\{x_{1}>0\right\} \cap\{t<0\} .
$$

The nondegeneracy assumption of $u$ at $\left(x_{0}, t_{0}\right)$ implies that necessarily $\alpha>0$.

Let us now show that $\alpha=\sqrt{2 M}$. By Lemma 4.3 there exists a subsequence $\varepsilon_{j_{n}}$ such that $\delta_{n}:=\frac{\varepsilon_{j_{n}}}{\lambda_{n}} \rightarrow 0$ and $u^{\delta_{n}}(x, t):=\frac{1}{\lambda_{n}} u^{\varepsilon_{j_{n}}}\left(\lambda_{n} x, \lambda_{n}^{2} t\right) \rightarrow U(x, t)$ uniformly on compact sets of $\mathbb{R}^{N+1}$. Let $f^{\delta_{n}}(x, t):=\lambda_{n} f^{\varepsilon_{j_{n}}}\left(\lambda_{n} x, \lambda_{n}^{2} t\right)$. Then, $f^{\delta_{n}} \rightarrow 0$ uniformly on compact sets of $\mathbb{R}^{N+1}$ and $u^{\delta_{n}}$ is a solution to $P_{\delta_{n}}\left(f^{\delta_{n}}\right)$.

Now let $U_{\lambda}(x, t)=\frac{1}{\lambda} U\left(\lambda x, \lambda^{2} t\right)$. Then for a sequence $\lambda_{k} \rightarrow 0, U_{\lambda_{k}} \rightarrow \alpha x_{1}^{+}$in $\{t \leq 0\}$, uniformly on compact subsets.

As before, there exists a subsequence $\delta_{n_{k}}$ such that $\bar{\delta}_{k}:=\frac{\delta_{n_{k}}}{\lambda_{k}} \rightarrow 0$ and that $u^{\bar{\delta}_{k}}(x, t):=$ $\frac{1}{\lambda_{k}} u^{\delta_{n_{k}}}\left(\lambda_{k} x, \lambda_{k}^{2} t\right)$ satisfies that $u^{\bar{\delta}_{k}} \rightarrow \alpha x_{1}^{+}$in $\{t \leq 0\}$, uniformly on compact subsets.

Since $u^{\bar{\delta}_{k}}$ is a solution to $P_{\bar{\delta}_{k}}\left(\bar{f}^{\bar{\delta}_{k}}\right)$ with $\bar{f}^{\bar{\delta}_{k}} \rightarrow 0$ (they are rescalings of the functions $f^{\delta_{n_{k}}}$ ) uniformly on compact sets of $\mathbb{R}^{N+1}$, we may apply Proposition 5.1 and deduce that $\alpha=\sqrt{2 M}$. 
Let us finally see that $U=\sqrt{2 M} x_{1}^{+}$in $\{t \leq 0\}$. In fact, $U \geq 0, \partial\{U>0\} \neq \emptyset$ and thus by Theorem 6.2 in [7] (see comment after Theorem 6.1) we have $|\nabla U| \leq \sqrt{2 M}$. Using that $U \equiv 0$ in $\left\{x_{1}=0\right\} \cap\{t \leq 0\}$ we deduce that

$$
U \leq \sqrt{2 M} x_{1} \quad \text { in }\left\{x_{1}>0\right\} \cap\{t \leq 0\} .
$$

Since $U$ is globally subcaloric and satisfies (7.3) with $\alpha=\sqrt{2 M}$, the application of Hopf's Principle yields that the equality holds in (7.4).

In the following corollary we use Hopf's Principle for caloric functions at boundary points where a parabolic Dini condition is satisfied (see [12]).

Corollary 7.1. Let $u^{\varepsilon_{j}}$ be nonnegative solutions to $P_{\varepsilon_{j}}\left(f^{\varepsilon_{j}}\right)$ in a domain $\mathcal{D} \subset \mathbb{R}^{N+1}$ such that $u^{\varepsilon_{j}}$ converge to $u$ uniformly on compact subsets of $\mathcal{D}, f^{\varepsilon_{j}} \rightarrow f *$-weakly in $L^{\infty}(\mathcal{D})$ and $\varepsilon_{j} \rightarrow 0$. Let $\left(x_{0}, t_{0}\right) \in \mathcal{D} \cap \partial\{u>0\}$ be a regular point. Assume that one of the following conditions is satisfied:

i) $f$ is nonpositive in a neighborhood of $\left(x_{0}, t_{0}\right)$ and $\partial\{u>0\}$ satisfies a Dini interior condition at $\left(x_{0}, t_{0}\right)$

ii) $\frac{u^{\varepsilon_{j}}}{\varepsilon_{j}} \rightarrow 0$ a.e. in $\mathcal{D} \cap\{u \equiv 0\} \cap\left\{t \leq t_{0}\right\}$.

Then the same conclusion of Theorem 7.1 holds.

Proof. Assume i). Then, there holds that $\Delta u-u_{t}=f$ in $\{u>0\}$ with $f \leq 0$ in a neighborhood of $\left(x_{0}, t_{0}\right)$. From Hopf's Principle (see [12]) we deduce that $u$ is nondegenerate at $\left(x_{0}, t_{0}\right)$ and thus the result follows from Theorem 7.1.

In case ii) holds, the nondegeneracy follows with arguments similar to those of Thm. 6.2.

Theorem 7.2. Let $u^{\varepsilon_{j}}$ be nonnegative solutions to $P_{\varepsilon_{j}}\left(f^{\varepsilon_{j}}\right)$ in a domain $\mathcal{D} \subset \mathbb{R}^{N+1}$ such that $u^{\varepsilon_{j}}$ converge to $u$ uniformly on compact subsets of $\mathcal{D}, f^{\varepsilon_{j}} \rightarrow f *$-weakly in $L^{\infty}(\mathcal{D})$ and $\varepsilon_{j} \rightarrow 0$. Let $\left(x_{0}, t_{0}\right) \in \mathcal{D} \cap \partial\{u>0\}$ be such that there exists $\nu \in \mathbb{R}^{N},|\nu|=1$, that satisfies (7.2) with $Q_{r}^{-}\left(x_{0}, t_{0}\right)$ replaced by $Q_{r}\left(x_{0}, t_{0}\right)$. Assume that $\frac{u^{\varepsilon_{j}}}{\varepsilon_{j}} \rightarrow 0$ a.e. in $\mathcal{D} \cap\{u \equiv 0\}^{\circ}$. Then, for every $t$,

$$
u(x, t)=\sqrt{2 M}\left\langle x-x_{0}, \nu\right\rangle^{+}+o\left(\left|x-x_{0}\right|+\left|t-t_{0}\right|^{\frac{1}{2}}\right) .
$$

Proof. From our assumptions it follows that $B_{\varepsilon_{j}}\left(u^{\varepsilon_{j}}\right) \rightarrow M \chi_{\{u>0\}}$ in $L_{\text {loc }}^{1}(\mathcal{D})$. Proceeding as in Theorem 3.1 in [8] we deduce that $u_{\lambda}(x, t)=\frac{1}{\lambda} u\left(x_{0}+\lambda x, t_{0}+\lambda^{2} t\right)$ verifies that, for every $\psi \in C_{0}^{\infty}\left(\mathbb{R}^{N+1}\right)$,

$$
\begin{aligned}
\iint\left(u_{\lambda}\right)_{t}\left(u_{\lambda}\right)_{x_{1}} \psi & =\frac{1}{2} \iint\left|\nabla u_{\lambda}\right|^{2} \psi_{x_{1}}-\iint\left(u_{\lambda}\right)_{x_{1}} \nabla u_{\lambda} \nabla \psi \\
& +M \iint_{\left\{u_{\lambda}>0\right\}} \psi_{x_{1}}+\lambda \iint f\left(x_{0}+\lambda x, t_{0}+\lambda^{2} t\right)\left(u_{\lambda}\right)_{x_{1}} \psi .
\end{aligned}
$$

Since the last term in the right hand side of (7.5) goes to zero as $\lambda \rightarrow 0$, the proof follows as in [8], with arguments similar to those in the proof of Theorem 7.1.

\section{Viscosity SOLUTIONS TO THE FREE BOUNDARY PROBLEM}

In this section we consider $u=\lim u^{\varepsilon}$, with $u^{\varepsilon}$ solutions to problems $P_{\varepsilon}\left(f^{\varepsilon}\right)$, and we prove that $u=\lim u^{\varepsilon}$ is a solution of the free boundary problem $P(f)$ in a viscosity sense (Corollaries 8.1 and 8.2), under a nondegeneracy assumption - which is necessary.

First, we prove results on asymptotic developments at free boundary points in which there is a tangent ball contained either in $\{u>0\}$ or in $\{u \equiv 0\}$, that are new even for (1.2) (Theorems 
8.1 and 8.2). The corollaries follow as an immediate consequence. The proofs of Theorems 8.1 and 8.2 make strong use of general results on asymptotic developments that we prove in the Appendix.

We start the section with some definitions:

Definition 8.1. Let $u$ be a continuous nonnegative function in a domain $\mathcal{D} \subset \mathbb{R}^{N+1}$. We say that a point $\left(x_{0}, t_{0}\right) \in \mathcal{D} \cap \partial\{u>0\}$ is a regular point from the right if there is a tangent ball at $\left(x_{0}, t_{0}\right)$ from $\mathcal{D} \cap\{u>0\}$ for $t \leq t_{0}$ (i.e, there is a ball $B$ such that $B \cap\left\{t \leq t_{0}\right\} \subset\{u>0\}$, with $\left.\left(x_{0}, t_{0}\right) \in \partial B\right)$, such that the spatial normal to $\partial B$ at $\left(x_{0}, t_{0}\right)$ does not vanish.

Analogously, we say that a point $\left(x_{0}, t_{0}\right) \in \mathcal{D} \cap \partial\{u>0\}$ is a regular point from the left if there is a tangent ball at $\left(x_{0}, t_{0}\right)$ from $\mathcal{D} \cap\{u \equiv 0\}^{\circ}$ for $t \leq t_{0}$ (i.e, there is a ball $B$ such that $B \cap\left\{t \leq t_{0}\right\} \subset\{u \equiv 0\}^{\circ}$, with $\left.\left(x_{0}, t_{0}\right) \in \partial B\right)$, such that the spatial normal to $\partial B$ at $\left(x_{0}, t_{0}\right)$ does not vanish.

Definition 8.2. Let $u$ be a nonnegative continuous function in a domain $\mathcal{D} \subset \mathbb{R}^{N+1}$. Let $f \in L^{\infty}(\mathcal{D})$. Then $u$ is called a viscosity supersolution of $P(f)$ in $\mathcal{D}$ if

(i) $\Delta u-u_{t} \leq f \quad$ in $\mathcal{D} \cap\{u>0\}$.

(ii) Along $\mathcal{D} \cap \partial\{u>0\}$, $u$ satisfies the condition $u_{\nu}^{+} \leq \sqrt{2 M}$ in the following weak sense. If $\left(x_{0}, t_{0}\right) \in \mathcal{D} \cap \partial\{u>0\}$ is a regular point from the right with touching ball $B$ and

$$
u(x, t) \geq \alpha\left\langle x-x_{0}, \nu\right\rangle^{+}+o\left(\left|x-x_{0}\right|+\left|t-t_{0}\right|^{\frac{1}{2}}\right), \quad \text { in } B \cap\left\{t \leq t_{0}\right\},
$$

with $\nu$ the inward unit spatial normal to $\partial B$ at $\left(x_{0}, t_{0}\right)$, then $\alpha \leq \sqrt{2 M}$.

Definition 8.3. Let $u$ be a nonnegative continuous function in a domain $\mathcal{D} \subset \mathbb{R}^{N+1}$. Let $f \in L^{\infty}(\mathcal{D})$. Then $u$ is called a viscosity subsolution of $P(f)$ in $\mathcal{D}$ if

(i) $\Delta u-u_{t} \geq f \quad$ in $\mathcal{D} \cap\{u>0\}$.

(ii) Along $\mathcal{D} \cap \partial\{u>0\}$, $u$ satisfies the condition $u_{\nu}^{+} \geq \sqrt{2 M}$ in the following weak sense. If $\left(x_{0}, t_{0}\right) \in \mathcal{D} \cap \partial\{u>0\}$ is a regular point from the left with touching ball $B$, then

$$
u(x, t)>\alpha\left\langle x-x_{0}, \nu\right\rangle^{+}+o\left(\left|x-x_{0}\right|+\left|t-t_{0}\right|^{\frac{1}{2}}\right) \quad \text { in } \mathcal{C} B \cap\left\{t \leq t_{0}\right\},
$$

for any $\alpha<\sqrt{2 M}$, with $\nu$ the outward unit spatial normal to $\partial B$ at $\left(x_{0}, t_{0}\right)$.

Definition 8.4. We say that $u$ is a viscosity solution of $P(f)$ in a domain $\mathcal{D} \subset \mathbb{R}^{N+1}$ if it is both a viscosity subsolution and a viscosity supersolution of $P(f)$ in $\mathcal{D}$.

We obtain

Theorem 8.1. Let $u^{\varepsilon_{j}}$ be nonnegative solutions to $P_{\varepsilon_{j}}\left(f^{\varepsilon_{j}}\right)$ in a domain $\mathcal{D} \subset \mathbb{R}^{N+1}$ such that $u^{\varepsilon_{j}} \rightarrow u$ uniformly on compact subsets of $\mathcal{D}, f^{\varepsilon_{j}} \rightarrow f *$-weakly in $L^{\infty}(\mathcal{D})$ and $\varepsilon_{j} \rightarrow 0$. Let $\left(x_{0}, t_{0}\right) \in \mathcal{D} \cap \partial\{u>0\}$ be a regular point from the right with touching ball $B$. Then, $u$ has the following asymptotic development

$$
u(x, t)=\bar{\alpha}\left\langle x-x_{0}, \nu\right\rangle^{+}+o\left(\left|x-x_{0}\right|+\left|t-t_{0}\right|^{\frac{1}{2}}\right), \quad \text { in } B \cap\left\{t \leq t_{0}\right\},
$$

with $0 \leq \bar{\alpha} \leq \sqrt{2 M}$, where $\nu$ is the inward unit spatial normal to $\partial B$ at $\left(x_{0}, t_{0}\right)$.

Proof. Let us assume that $\left(x_{0}, t_{0}\right)=(0,0)$ and the inward unit spatial normal to $\partial B$ is $e_{1}$.

By Lemma A.1 in the appendix there exists $\bar{\alpha} \geq 0$ such that

$$
u(x, t)=\bar{\alpha} x_{1}^{+}+o\left(|x|+|t|^{\frac{1}{2}}\right) \quad \text { in } B \cap\{t \leq 0\} .
$$

So we only have to prove that $\bar{\alpha} \leq \sqrt{2 M}$.

If $\bar{\alpha}=0$ there is nothing to prove. So let us assume that $\bar{\alpha}>0$. 
Let $u_{\lambda}(x, t)=\frac{1}{\lambda} u\left(\lambda x, \lambda^{2} t\right)$. Then, for a subsequence $\lambda_{n} \rightarrow 0$, there holds that $u_{\lambda_{n}} \rightarrow u_{0} \geq 0$ uniformly on compact subsets of $\mathbb{R}^{N+1}$ for a function $u_{0} \in \operatorname{Lip}(1,1 / 2)$ with $u_{0}(x, t)=\bar{\alpha} x_{1}^{+}$in $\left\{x_{1}>0\right\}$ and $u_{0}$ a solution of the homogeneous heat equation in $\left\{x_{1}<0\right\} \cap\left\{u_{0}>0\right\}$ with $u_{0}=0$ on $\left\{x_{1}=0\right\}$.

Thus, applying Corollary A.1 of [7], we find that

$$
u_{0}=\gamma x_{1}^{-}+o\left(|x|+|t|^{\frac{1}{2}}\right) \quad \text { in }\left\{x_{1}<0\right\}, \quad \text { with } \gamma \geq 0 .
$$

On the other hand, there exist $\varepsilon_{j_{n}}$ such that $\delta_{n}:=\frac{\varepsilon_{j_{n}}}{\lambda_{n}} \rightarrow 0$ and $u^{\delta_{n}}=\frac{1}{\lambda_{n}} u^{\varepsilon_{j_{n}}}\left(\lambda_{n} x, \lambda_{n}^{2} t\right) \rightarrow$ $u_{0} \quad(n \rightarrow \infty)$. Let $f^{\delta_{n}}(x, t):=\lambda_{n} f^{\varepsilon_{j_{n}}}\left(\lambda_{n} x, \lambda_{n}^{2} t\right)$. Then, $f^{\delta_{n}} \rightarrow 0$ uniformly on compact subsets of $\mathbb{R}^{N+1}$ and $u^{\delta_{n}}$ is a solution to $P_{\delta_{n}}\left(f^{\delta_{n}}\right)$.

Now let $u_{00}=\lim \left(u_{0}\right)_{\lambda_{k}}$ for a sequence $\lambda_{k} \rightarrow 0$. Then, $u_{00}=\bar{\alpha} x_{1}^{+}+\gamma x_{1}^{-}$. As before, there exists a subsequence $\delta_{n_{k}}$ such that $\bar{\delta}_{k}:=\frac{\delta_{n_{k}}}{\lambda_{k}} \rightarrow 0$ and $u_{00}=\lim _{k \rightarrow \infty} u^{\bar{\delta}_{k}}$.

Arguing as in Theorem 7.1, we may apply Proposition 5.1 if $\gamma=0$ or Proposition 5.2 if $\gamma>0$. In either case we deduce that $\bar{\alpha} \leq \sqrt{2 M}$.

Theorem 8.2. Let $u^{\varepsilon_{j}}$ be nonnegative solutions to $P_{\varepsilon_{j}}\left(f^{\varepsilon_{j}}\right)$ in a domain $\mathcal{D} \subset \mathbb{R}^{N+1}$ such that $u^{\varepsilon_{j}} \rightarrow u$ uniformly on compact subsets of $\mathcal{D}, f^{\varepsilon_{j}} \rightarrow f *$-weakly in $L^{\infty}(\mathcal{D})$ and $\varepsilon_{j} \rightarrow 0$. Let $\left(x_{0}, t_{0}\right) \in \mathcal{D} \cap \partial\{u>0\}$ be a regular point from the left with touching ball $B$ and assume that $u$ is nondegenerate at $\left(x_{0}, t_{0}\right)$. Then, $u$ has the following asymptotic development

$$
u(x, t)=\sqrt{2 M}\left\langle x-x_{0}, \nu\right\rangle^{+}+o\left(\left|x-x_{0}\right|+\left|t-t_{0}\right|^{\frac{1}{2}}\right), \quad \text { in }\left\{t \leq t_{0}\right\},
$$

where $\nu$ is the outward unit spatial normal to $\partial B$ at $\left(x_{0}, t_{0}\right)$.

Proof. Let us assume that $\left(x_{0}, t_{0}\right)=(0,0)$ and $\nu=e_{1}$. By Lemma A.2 in the appendix, there exists $\alpha \geq 0$ such that

$$
u(x, t)=\alpha x_{1}^{+}+o\left(|x|+|t|^{\frac{1}{2}}\right) \quad \text { in } \mathcal{C} B \cap\{t \leq 0\} .
$$

We want to prove that $\alpha=\sqrt{2 M}$.

First, $\alpha>0$ since, by hypothesis, $u$ is nondegenerate at $(0,0)$.

We perform a blow up as in the proof of Theorem 8.1 and find a sequence $\delta_{n} \rightarrow 0$ such that $u^{\delta_{n}} \rightarrow u_{0}=\alpha x_{1}^{+}$uniformly on compact sets of $\mathbb{R}^{N+1}$ with $u^{\delta_{n}}$ solutions to $P_{\delta_{n}}\left(f^{\delta_{n}}\right)$ and $f^{\delta_{n}} \rightarrow 0$ uniformly. By Proposition 5.1 we deduce that $\alpha=\sqrt{2 M}$.

As a corollary we obtain

Corollary 8.1. Let $u^{\varepsilon_{j}}$ be nonnegative solutions to $P_{\varepsilon_{j}}\left(f^{\varepsilon_{j}}\right)$ in a domain $\mathcal{D} \subset \mathbb{R}^{N+1}$ such that $u^{\varepsilon_{j}} \rightarrow u$ uniformly on compact subsets of $\mathcal{D}, f^{\varepsilon_{j}} \rightarrow f *$-weakly in $L^{\infty}(\mathcal{D})$ and $\varepsilon_{j} \rightarrow 0$. Then $u$ is a viscosity supersolution to $P(f)$ in $\mathcal{D}$.

Corollary 8.2. Let $u^{\varepsilon_{j}}$ be nonnegative solutions to $P_{\varepsilon_{j}}\left(f^{\varepsilon_{j}}\right)$ in a domain $\mathcal{D} \subset \mathbb{R}^{N+1}$ such that $u^{\varepsilon_{j}} \rightarrow u$ uniformly on compact subsets of $\mathcal{D}, f^{\varepsilon_{j}} \rightarrow f *$-weakly in $L^{\infty}(\mathcal{D})$ and $\varepsilon_{j} \rightarrow 0$. Assume that $u$ is nondegenerate at every regular point from the left in $\mathcal{D} \cap \partial\{u>0\}$. Then $u$ is a viscosity subsolution to $P(f)$ in $\mathcal{D}$.

Remark 8.1. In this paper we have chosen a definition of viscosity solution that differs from the one in [8]. It is easy to see that our viscosity solutions are also viscosity solutions in the sense of [8] if the comparison classical sub and supersolutions are taken with $C^{2}$ free boundaries.

On the other hand, we have chosen this definition because we consider that it is more suitable for the nonlocal problem (see Section 9, Theorems 9.2 and 9.3). 


\section{The NONLOCAL PROBLEM}

We consider the nonlocal problem (2.4), where the kernel $J$ is as in Section 1.

In Section 2 (Theorem 2.1) we proved existence, uniqueness and uniform $L^{\infty}$ estimates of the solution of problem $(2.4)$ when $\left\|u_{0}^{\varepsilon}\right\|_{L^{\infty}\left(\mathbb{R}^{N}\right)} \leq \mathcal{A}_{1}$ and $\left\|g^{\varepsilon}\right\|_{L^{\infty}\left(\mathbb{R}^{N} \times(0, T)\right)} \leq \mathcal{A}_{2}$ for some $\mathcal{A}_{1}>0$, $\mathcal{A}_{2}>0$. This solution is nonnegative if, for instance, $u_{0}^{\varepsilon} \geq 0$ in $\mathbb{R}^{N}$ and $g^{\varepsilon} \leq 0$ in $\mathbb{R}^{N} \times(0, T)$.

Then, as in Section 3 we define

$$
\bar{u}^{\varepsilon}(x, t)=u^{\varepsilon}(\sqrt{\theta} x, t), \quad f^{\varepsilon}(x, t)=-(1-\theta)\left(J * u^{\varepsilon}-u^{\varepsilon}\right)(\sqrt{\theta} x, t)+g^{\varepsilon}(\sqrt{\theta} x, t),
$$

so that $\bar{u}^{\varepsilon}$ are uniformly bounded solutions to $P_{\varepsilon}\left(f^{\varepsilon}\right)$ in $\mathbb{R}^{N} \times(0, T)$, with $f^{\varepsilon}$ uniformly bounded.

Thus we can apply the results of Sections 4 to 8 to the family $\bar{u}^{\varepsilon}$. In particular, for a subsequence, $u^{\varepsilon}$ converges uniformly on compact sets to a nonnegative function $u \in \operatorname{Lip}_{\text {loc }}(1,1 / 2)$ satisfying

$$
\theta \Delta u-u_{t}+[(1-\theta)(J * u-u)-g] \chi_{\{u>0\}}=\Lambda
$$

where $\Lambda$ is a nonnegative Radon measure supported on $\partial\{u>0\}$. Here $g=\lim g^{\varepsilon} *$-weakly.

With respect to the characterization of the measure $\Lambda$ our main results are:

Theorem 9.1. Let $u^{\varepsilon_{j}}$ be nonnegative solutions to the nonlocal problem (2.4) in $\mathbb{R}^{N} \times(0, T)$ such that $u^{\varepsilon_{j}}$ converge to $u$ uniformly on compact subsets of $\mathbb{R}^{N} \times(0, T], g^{\varepsilon_{j}} \rightarrow g$ *-weakly in $L^{\infty}\left(\mathbb{R}^{N} \times(0, T)\right)$ and $\varepsilon_{j} \rightarrow 0$. Let $\left(x_{0}, t_{0}\right) \in \partial\{u>0\} \cap\{0<t<T\}$ be a regular point. Assume that $u$ is nondegenerate at $\left(x_{0}, t_{0}\right)$. Then, for $t \leq t_{0}$,

$$
u(x, t)=\sqrt{\frac{2 M}{\theta}}\left\langle x-x_{0}, \nu\right\rangle^{+}+o\left(\left|x-x_{0}\right|+\left|t-t_{0}\right|^{\frac{1}{2}}\right)
$$

where $\nu$ is the inward unit spatial normal to $\partial\{u>0\}$ at $\left(x_{0}, t_{0}\right)$, for $t \leq t_{0}$, in the parabolic measure theoretic sense.

As a corollary we have

Corollary 9.1. Let $u^{\varepsilon_{j}}$ be nonnegative solutions to the nonlocal problem (2.4) in $\mathbb{R}^{N} \times(0, T)$ such that $u^{\varepsilon_{j}}$ converge to $u$ uniformly on compact subsets of $\mathbb{R}^{N} \times(0, T], g^{\varepsilon_{j}} \rightarrow g$ *-weakly in $L^{\infty}\left(\mathbb{R}^{N} \times(0, T)\right)$ and $\varepsilon_{j} \rightarrow 0$. Let $\left(x_{0}, t_{0}\right) \in \partial\{u>0\} \cap\{0<t<T\}$ be a regular point. Assume that one of the following conditions is satisfied:

i) The kernel $J$ is positive in a neighborhood of the origin, $g$ is nonpositive in a neighborhood of $\left(x_{0}, t_{0}\right)$ and $\partial\{u>0\}$ satisfies a Dini interior condition at $\left(x_{0}, t_{0}\right)$

ii) $\frac{u^{\varepsilon_{j}}}{\varepsilon_{j}} \rightarrow 0$ a.e. in $\mathcal{D} \cap\{u \equiv 0\} \cap\left\{t \leq t_{0}\right\}$.

Then the same conclusion of Theorem 9.1 holds.

Proof. In case i) holds we have

$$
\theta \Delta u^{\varepsilon_{j}}-u_{t}^{\varepsilon_{j}}=\beta_{\varepsilon_{j}}\left(u^{\varepsilon_{j}}\right)-(1-\theta)\left(J * u^{\varepsilon_{j}}-u^{\varepsilon_{j}}\right)+g^{\varepsilon_{j}},
$$

and since in the present situation there holds $u\left(x_{0}, t_{0}\right)=0,(J * u)\left(x_{0}, t_{0}\right)>0$ and $g\left(x_{0}, t_{0}\right) \leq 0$, we see that the limit in the forcing term in (9.2) is nonpositive in a neighborhood of $\left(x_{0}, t_{0}\right)$.

In any case, Corollary 7.1 applies to $\bar{u}^{\varepsilon_{j}}(x, t)=u^{\varepsilon_{j}}(\sqrt{\theta} x, t)$.

In particular this result implies, when the free boundary is smooth and $g \leq 0$, that

$$
\Lambda=\sqrt{2 M \theta} \mathcal{H}^{N}\left\lfloor\partial\{u>0\} \quad \text { and } \quad|\nabla u|=\sqrt{\frac{2 M}{\theta}} \quad \text { on } \partial\{u>0\} .\right.
$$

From the results in Section 8 we deduce 
Theorem 9.2. Let $u^{\varepsilon_{j}}$ be nonnegative solutions to the nonlocal problem (2.4) in $\mathbb{R}^{N} \times(0, T)$ such that $u^{\varepsilon_{j}}$ converge to $u$ uniformly on compact subsets of $\mathbb{R}^{N} \times(0, T], g^{\varepsilon_{j}} \rightarrow g$ *-weakly in $L^{\infty}\left(\mathbb{R}^{N} \times(0, T)\right)$ and $\varepsilon_{j} \rightarrow 0$. Then, $u$ satisfies

$$
\theta \Delta u+(1-\theta)(J * u-u)-u_{t}=g \quad \text { in }\{u>0\} .
$$

Let $\left(x_{0}, t_{0}\right) \in \partial\{u>0\} \cap\{0<t<T\}$. If $\left(x_{0}, t_{0}\right)$ is a regular point from the right with touching ball $B$ then, in $B \cap\left\{t \leq t_{0}\right\}$, $u$ has the asymptotic development

$$
u(x, t)=\alpha\left\langle x-x_{0}, \nu\right\rangle^{+}+o\left(\left|x-x_{0}\right|+\left|t-t_{0}\right|^{\frac{1}{2}}\right),
$$

with $0 \leq \alpha \leq \sqrt{\frac{2 M}{\theta}}$ and $\nu$ the inward unit spatial normal to $\partial B$ at $\left(x_{0}, t_{0}\right)$.

If $\left(x_{0}, t_{0}\right)$ is a regular point from the left with touching ball $B$ then, in $\left\{t \leq t_{0}\right\}, u$ has the asymptotic development (9.3), with $\alpha=0$ or $\alpha=\sqrt{\frac{2 M}{\theta}}$ and $\nu$ the outward unit spatial normal to $\partial B$ at $\left(x_{0}, t_{0}\right)$.

We also obtain

Theorem 9.3. Let $u^{\varepsilon_{j}}$ be nonnegative solutions to the nonlocal problem (2.4) in $\mathbb{R}^{N} \times(0, T)$ such that $u^{\varepsilon_{j}}$ converge to $u$ uniformly on compact subsets of $\mathbb{R}^{N} \times(0, T], g^{\varepsilon_{j}} \rightarrow g$ *-weakly in $L^{\infty}\left(\mathbb{R}^{N} \times(0, T)\right)$ and $\varepsilon_{j} \rightarrow 0$. Let $\bar{u}=\lim \bar{u}^{\varepsilon_{j}}$ and $f=\lim f^{\varepsilon_{j}}\left(\bar{u}^{\varepsilon_{j}}\right.$ and $f^{\varepsilon_{j}}$ as in (9.1)) and assume that $\bar{u}$ is nondegenerate at every regular point from the left in $\mathcal{D} \cap \partial\{\bar{u}>0\}$, for some domain $\mathcal{D} \subset \mathbb{R}^{N} \times(0, T)$. Then $\bar{u}$ is a viscosity solution to $P(f)$ in $\mathcal{D}$.

The results in Section 3 provide examples of stationary solutions to the nonlocal problem (2.4). We next present other examples of solutions to this nonlocal problem. We first show that, under suitable assumptions, if $u=\lim u^{\varepsilon}$ has bounded initial support, then the support (and thus the free boundary) remains bounded and moreover, it shrinks.

Proposition 9.1. Let $u_{0} \in C\left(\mathbb{R}^{N}\right) \cap C^{2}\left(\overline{\left\{u_{0}>0\right\}}\right)$ be a nonnegative function with compact support. Let $g \in C\left(\mathbb{R}^{N} \times[0, T]\right) \cap L^{\infty}\left(\mathbb{R}^{N} \times(0, T)\right)$ be such that $g \leq 0$ and $g$ is nondecreasing in t. Assume that $\theta \Delta u_{0}+(1-\theta)\left(J * u_{0}-u_{0}\right)-g(x, 0) \leq-\rho_{0}$ in $\left\{u_{0}>0\right\}$ for some $\rho_{0}>0$ and that $0<\left|\nabla u_{0}\right| \leq a_{1}<\sqrt{\frac{2 M}{\theta}}$ on $\partial\left\{u_{0}>0\right\}$.

Let $g^{\varepsilon} \in C\left(\mathbb{R}^{N} \times[0, T]\right)$ be a uniformly bounded family such that $g^{\varepsilon} \leq 0$, $g^{\varepsilon}$ are nondecreasing in $t$ and $g^{\varepsilon} \rightarrow g$ uniformly in $\mathbb{R}^{N} \times[0, T]$. Then, there exists a $L^{\infty}$-uniformly bounded family $u_{0}^{\varepsilon} \in C^{1}\left(\mathbb{R}^{N}\right)$ with $u_{0}^{\varepsilon} \geq 0$ and $u_{0}^{\varepsilon} \rightarrow u_{0}$ uniformly in $\mathbb{R}^{N}$ such that, if $\varepsilon$ is small enough,

$$
\theta \Delta u_{0}^{\varepsilon}+(1-\theta)\left(J * u_{0}^{\varepsilon}-u_{0}^{\varepsilon}\right)-g^{\varepsilon}(x, 0)-\beta_{\varepsilon}\left(u_{0}^{\varepsilon}\right) \leq-\frac{1}{2} \rho_{0} \quad \text { in } \mathbb{R}^{N} .
$$

Now let $u^{\varepsilon} \in C\left(\mathbb{R}^{N} \times[0, T]\right) \cap L^{\infty}\left(\mathbb{R}^{N} \times(0, T)\right)$ be the solution to (2.4) with $u_{0}^{\varepsilon}$ and $g^{\varepsilon}$ as above (so that, in particular, $u^{\varepsilon}$ are nonnegative and uniformly bounded). Then, $u^{\varepsilon}$ is nonincreasing in $t, 0 \leq t \leq T$. Thus, if $\varepsilon_{j} \rightarrow 0$ and $u=\lim u^{\varepsilon_{j}}$, the supports $\overline{\{u(\cdot, t)>0\}}$ are bounded and nonincreasing, $0 \leq t \leq T$.

Proof. Our construction of $u_{0}^{\varepsilon}$ will be a modification of the construction of the initial data in [9]. The approximations are defined in the following way. First we modify $u_{0}$ where it vanishes, in such a way that in a neighborhood $S$ of $\left\{u_{0}>0\right\}, S:=\left\{x \in \mathbb{R}^{N} / \operatorname{dist}\left(x,\left\{u_{0}>0\right\}\right) \leq \gamma\right\}$, we have $\left\|u_{0}\right\|_{C^{2}(S)}<\infty$. We can assume that $0<a_{2} \leq\left|\nabla u_{0}\right| \leq a_{1}<\sqrt{\frac{2 M}{\theta}}$ if $\operatorname{dist}\left(x, \partial\left\{u_{0}>0\right\}\right) \leq$ $\gamma$ 
For $\varepsilon$ small we consider $F_{\varepsilon} \in C^{2}(\mathbb{R})$ such that

$$
\theta F_{\varepsilon}^{\prime \prime} \leq(1+\delta) \beta\left(F_{\varepsilon}\right)+\alpha F_{\varepsilon}^{\prime}-\varepsilon D, \quad F_{\varepsilon}(0)=1, \quad F_{\varepsilon}^{\prime}(0)=-\sqrt{\frac{2 M}{\theta}} .
$$

Here $D, \delta$ and $\alpha$ are positive constants ( $D$ big and $\delta$ small to be fixed later) and $F_{\varepsilon}$ is such that it has a strict minimum at a point $\bar{s}_{\varepsilon}$ with $\sqrt{\frac{\theta}{2 M}}<\bar{s}_{\varepsilon}<\bar{s}$ and $0<c<F_{\varepsilon}\left(\bar{s}_{\varepsilon}\right)<1-c(c, \bar{s}$ independent of $\varepsilon$ ) and $F_{\varepsilon}$ is decreasing for $s<\bar{s}_{\varepsilon}$.

The existence of such a $F_{\varepsilon}$, when $\alpha=\varepsilon=0$ follows, for instance, from the analysis in Propositions 3.1 and 3.2 and it thus follows by continuous dependence for $\alpha$ small and $\varepsilon \leq \varepsilon_{0}$.

We let $C_{\varepsilon}=\bar{s}_{\varepsilon} \sqrt{\frac{2 M}{\theta}}-1$ and we define, for $\varepsilon$ small,

$$
\begin{aligned}
& u_{0}^{\varepsilon}(x)=u_{0}(x) \quad \text { in }\left\{u_{0}>\varepsilon\right\}, \\
& u_{0}^{\varepsilon}(x)=\varepsilon F_{\varepsilon}\left(\sqrt{\frac{\theta}{2 M}}\left(1-\frac{u_{0}(x)}{\varepsilon}\right)\right) \quad \text { in } \quad\left\{x \in S /-C_{\varepsilon} \varepsilon \leq u_{0} \leq \varepsilon\right\}, \\
& u_{0}^{\varepsilon}(x)=\varepsilon F_{\varepsilon}\left(\bar{s}_{\varepsilon}\right) \quad \text { elsewhere. }
\end{aligned}
$$

As in [9], it follows that $u_{0}^{\varepsilon} \in C^{1}\left(\mathbb{R}^{N}\right)$.

I. Let us verify that (9.4) is satisfied in $\left\{u_{0}>\varepsilon\right\}$. In fact, if $\varepsilon+\left\|g^{\varepsilon}-g\right\|_{L^{\infty}} \leq \frac{\rho_{0}}{2}$,

$$
\begin{gathered}
\theta \Delta u_{0}^{\varepsilon}+(1-\theta)\left(J * u_{0}^{\varepsilon}-u_{0}^{\varepsilon}\right)-g^{\varepsilon}(x, 0)-\beta_{\varepsilon}\left(u_{0}^{\varepsilon}\right)=\theta \Delta u_{0}+(1-\theta)\left(J * u_{0}^{\varepsilon}-u_{0}\right)-g^{\varepsilon}(x, 0) \\
\leq-\rho_{0}+(1-\theta)\left(J * u_{0}^{\varepsilon}-J * u_{0}\right)+\left\|g^{\varepsilon}-g\right\|_{L^{\infty}} \leq-\frac{\rho_{0}}{2} .
\end{gathered}
$$

II. Let us see that (9.4) is satisfied in $\left\{x \in S /-C_{\varepsilon} \varepsilon \leq u_{0} \leq \varepsilon\right\}$. In fact,

$$
\begin{aligned}
\theta \Delta u_{0}^{\varepsilon} & +(1-\theta)\left(J * u_{0}^{\varepsilon}-u_{0}^{\varepsilon}\right)-g^{\varepsilon}(x, 0)-\beta_{\varepsilon}\left(u_{0}^{\varepsilon}\right) \\
& \leq \frac{1}{2 M \varepsilon} \theta^{2} F_{\varepsilon}^{\prime \prime}\left|\nabla u_{0}\right|^{2}-\sqrt{\frac{\theta}{2 M}} F_{\varepsilon}^{\prime} \theta \Delta u_{0}+B-\frac{1}{\varepsilon} \beta\left(F_{\varepsilon}\right) \\
& \leq \frac{(1+\delta) \theta}{2 M \varepsilon} \beta\left(F_{\varepsilon}\right)\left|\nabla u_{0}\right|^{2}+\frac{\alpha \theta}{2 M \varepsilon} F_{\varepsilon}^{\prime}\left|\nabla u_{0}\right|^{2}-\frac{\theta}{2 M} D\left|\nabla u_{0}\right|^{2}-a \sqrt{\frac{\theta}{2 M}} F_{\varepsilon}^{\prime}+B-\frac{1}{\varepsilon} \beta\left(F_{\varepsilon}\right),
\end{aligned}
$$

where $B=2\left(\left\|u_{0}\right\|_{L^{\infty}}+\|g\|_{L^{\infty}}\right)$ and $a>0$ is such that $\left|\Delta u_{0}\right| \leq a$ in $S$.

Let $0<A<1$ be such that $a_{1} \leq A \sqrt{\frac{2 M}{\theta}}$, and let $\delta$ and $D$ in the definition of $F_{\varepsilon}$ be such that $(1+\delta) A^{2} \leq 1$ and $D \geq \frac{2 M}{\theta a_{2}^{2}}\left(B+\frac{\rho_{0}}{2}\right)$. Then, if $\varepsilon$ is small enough so that $\alpha a_{2}^{2} \sqrt{\frac{\theta}{2 M}}>a \varepsilon$, there holds that

$$
\begin{aligned}
\theta \Delta u_{0}^{\varepsilon} & +(1-\theta)\left(J * u_{0}^{\varepsilon}-u_{0}^{\varepsilon}\right)-g^{\varepsilon}(x, 0)-\beta_{\varepsilon}\left(u_{0}^{\varepsilon}\right) \\
& \leq \frac{1}{\varepsilon}\left[\left[(1+\delta) A^{2}-1\right] \beta\left(F_{\varepsilon}\right)+\left(\frac{\alpha a_{2}^{2} \theta}{2 M}-a \varepsilon \sqrt{\frac{\theta}{2 M}}\right) F_{\varepsilon}^{\prime}\right]+\left(B-\frac{\theta D a_{2}^{2}}{2 M}\right) \leq-\frac{\rho_{0}}{2} .
\end{aligned}
$$

III. Let us finally show that (9.4) holds in the remainder of $\mathbb{R}^{N}$. We have

$$
\theta \Delta u_{0}^{\varepsilon}+(1-\theta)\left(J * u_{0}^{\varepsilon}-u_{0}^{\varepsilon}\right)-g^{\varepsilon}(x, 0)-\beta_{\varepsilon}\left(u_{0}^{\varepsilon}\right) \leq B-\frac{1}{\varepsilon} \beta\left(F_{\varepsilon}\left(\bar{s}_{\varepsilon}\right)\right) \leq B-\frac{\sigma}{\varepsilon} \leq-\frac{\rho_{0}}{2},
$$

if $\varepsilon\left(B+\frac{\rho_{0}}{2}\right) \leq \sigma$, where $\sigma=\min \{\beta(s) / c \leq s \leq 1-c\}$.

Now let $u^{\varepsilon} \in C\left(\mathbb{R}^{N} \times[0, T]\right) \cap L^{\infty}\left(\mathbb{R}^{N} \times(0, T)\right)$ be the solution to (2.4) with $u_{0}^{\varepsilon}$ and $g^{\varepsilon}$ as above. Then, by our previous results, $u^{\varepsilon}$ are nonnegative and uniformly bounded. 
On the other hand, for $\varepsilon$ small, $u_{t}^{\varepsilon}$ satisfies (in a weak sense)

$$
\begin{aligned}
\theta \Delta U+(1-\theta)(J * U-U)-U_{t} & =\beta_{\varepsilon}^{\prime}\left(u^{\varepsilon}\right) U+g_{t}^{\varepsilon} & \text { in } \mathbb{R}^{N} \times(0, T) \\
U(x, 0) & \leq-\frac{1}{2} \rho_{0}<0 & \text { in } \mathbb{R}^{N},
\end{aligned}
$$

with $g_{t}^{\varepsilon} \geq 0$.

Then, we approximate the initial data $u_{0}^{\varepsilon}$ uniformly in $\mathbb{R}^{N}$ by smooth functions $\left(u_{0}^{\varepsilon}\right)_{\delta}$ satisfying (9.4) with the right hand side $-\frac{1}{2} \rho_{0}$ replaced by $-\frac{1}{4} \rho_{0}$. Next we take $u_{\delta}^{\varepsilon}$ the solution to $(2.4)$ with initial data $\left(u_{0}^{\varepsilon}\right)_{\delta}$. Thus, by the results in Section 2 there holds that $u_{\delta}^{\varepsilon} \rightarrow u^{\varepsilon}$ uniformly on compact sets. As $\frac{\partial u_{\delta}^{\varepsilon}}{\partial t} \leq 0$ in $\mathbb{R}^{N} \times[0, T]$, there holds that $u^{\varepsilon}$ is nonincreasing in time.

The following result gives an example of choice of data $u_{0}^{\varepsilon}$ and $g^{\varepsilon}$ such that the solutions of the nonlocal problem (2.4) satisfy that $\bar{u}^{\varepsilon}$ meet the hypotheses of Theorem 9.3 , so that $\bar{u}$ is a viscosity solution to $P(f)$ in $\mathbb{R}^{N} \times(0, T)$.

Proposition 9.2. Assume that the kernel $J$ is positive in a neighborhood of the origin and satisfies that supp $J \subset B_{R}(0)$, for some $R>0$. Let $u_{0} \in C\left(\mathbb{R}^{N}\right) \cap C^{2}\left(\overline{\left\{u_{0}>0\right\}}\right)$ be a nonnegative function such that $\left\|u_{0}\right\|_{C^{2}(\overline{\{u>0\}})}<\infty$ and $\partial\left\{u_{0}>0\right\} \neq \emptyset$. Let $g \in C\left(\mathbb{R}^{N} \times[0, T]\right) \cap L^{\infty}\left(\mathbb{R}^{N} \times\right.$ $(0, T))$ be such that $g \leq 0$ and $g$ is nondecreasing in $t$. Assume that $\theta \Delta u_{0}+(1-\theta)\left(J * u_{0}-\right.$ $\left.u_{0}\right)-g(x, 0) \leq-\rho_{0} \chi_{B_{2 R}\left(\left\{u_{0} \equiv 0\right\}\right)}$ in $\left\{u_{0}>0\right\}$, for some $\rho_{0}>0$.

Assume moreover that $0<a_{2} \leq\left|\nabla u_{0}\right| \leq a_{1}<\sqrt{2 M / \theta}$ on $\partial\left\{u_{0}>0\right\}$ and that there exists a spatial cone $\Gamma$ with axis $e_{1}$ such that, in the directions of $\Gamma, u_{0}$ is nondecreasing and $g(\cdot, t)$ is nonincreasing, for every $t$.

There exist $L^{\infty}$-uniformly bounded families $u_{0}^{\varepsilon} \in C^{1}\left(\mathbb{R}^{N}\right)$ and $g^{\varepsilon} \in C\left(\mathbb{R}^{N} \times[0, T]\right)$, satisfying that $u_{0}^{\varepsilon} \geq 0, g^{\varepsilon} \leq 0, u_{0}^{\varepsilon} \rightarrow u_{0}$ uniformly in $\mathbb{R}^{N}$ and $g^{\varepsilon} \rightarrow g$ uniformly in $\mathbb{R}^{N} \times[0, T]$, such that if $u^{\varepsilon} \in C\left(\mathbb{R}^{N} \times[0, T]\right) \cap L^{\infty}\left(\mathbb{R}^{N} \times(0, T)\right)$ is the solution to (2.4) with this choice of $u_{0}^{\varepsilon}$ and $g^{\varepsilon}$ (so that, in particular, $u^{\varepsilon}$ are nonnegative and uniformly bounded) then $u^{\varepsilon}$ is under the hypotheses of Theorem 9.3 in $\mathbb{R}^{N} \times(0, T)$. Thus, if $\varepsilon_{j} \rightarrow 0, \bar{u}=\lim \bar{u}^{\varepsilon_{j}}$ and $f=\lim f^{\varepsilon_{j}}\left(\bar{u}^{\varepsilon_{j}}\right.$ and $f^{\varepsilon_{j}}$ as in (9.1)), then $\bar{u}$ is a viscosity solution to $P(f)$ in $\mathbb{R}^{N} \times(0, T)$.

Proof. We take the families $g^{\varepsilon}$ and $u_{0}^{\varepsilon}$ as in Prop. 9.1. Let us see that there holds in $\mathbb{R}^{N}$

$$
\theta \Delta u_{0}^{\varepsilon}+(1-\theta)\left(J * u_{0}^{\varepsilon}-u_{0}^{\varepsilon}\right)-g^{\varepsilon}(x, 0)-\beta_{\varepsilon}\left(u_{0}^{\varepsilon}\right) \leq-\frac{1}{2} \rho_{0} \chi_{\left\{x / \operatorname{dist}\left(x,\left\{u_{0} \equiv 0\right\}\right) \leq 2 R\right\}} .
$$

In fact, when $\operatorname{dist}\left(x,\left\{u_{0} \equiv 0\right\}\right) \leq 2 R,(9.5)$ follows by proceeding as in Prop. 9.1. Let us verify that (9.5) holds when $\operatorname{dist}\left(x,\left\{u_{0} \equiv 0\right\}\right)>2 R$. In this region, $u_{0}^{\varepsilon}=u_{0}>\varepsilon, J * u_{0}^{\varepsilon}=J * u_{0}$ (we use that supp $J \subset B_{R}(0)$ ). Then, (9.5) holds if we require that the approximations $g^{\varepsilon}$ verify that $g(x, 0) \leq g^{\varepsilon}(x, 0)$. Thus, as in Prop. 9.1, we see that $u^{\varepsilon}$ is nonincreasing in $t, 0 \leq t \leq T$.

On the other hand, the approximations $g^{\varepsilon}$ can be chosen satisfying in addition that

$$
g_{x_{1}}^{\varepsilon}+\lambda g_{x_{i}}^{\varepsilon} \leq 0, \quad g_{x_{1}}^{\varepsilon}-\lambda g_{x_{i}}^{\varepsilon} \leq 0,
$$

weakly, for every $i$ and for some constant $\lambda>0$. Let us see that, for every $i$, there holds that

$$
\left(u_{0}^{\varepsilon}\right)_{x_{1}}+\lambda\left(u_{0}^{\varepsilon}\right)_{x_{i}} \geq 0, \quad\left(u_{0}^{\varepsilon}\right)_{x_{1}}-\lambda\left(u_{0}^{\varepsilon}\right)_{x_{i}} \geq 0 .
$$

We only need to verify this property in $\left\{-C_{\varepsilon} \varepsilon<u_{0}<\varepsilon\right\}$ and this is clear from the fact that

$$
\nabla u_{0}^{\varepsilon}=-\sqrt{\frac{\theta}{2 M}} F_{\varepsilon}^{\prime}\left(\sqrt{\frac{\theta}{2 M}}\left(1-\frac{u_{0}}{\varepsilon}\right)\right) \nabla u_{0} .
$$


Since, for every $i, u_{x_{1}}^{\varepsilon}-\lambda u_{x_{i}}^{\varepsilon}$ satisfies (in a weak sense)

$$
\begin{aligned}
\theta \Delta U+(1-\theta)(J * U-U)-U_{t} & =\beta_{\varepsilon}^{\prime}\left(u^{\varepsilon}\right) U+g_{x_{1}}^{\varepsilon}-\lambda g_{x_{i}}^{\varepsilon} & \text { in } \mathbb{R}^{N} \times(0, T) \\
U(x, 0) & \geq 0 & \text { in } \mathbb{R}^{N},
\end{aligned}
$$

with $g_{x_{1}}^{\varepsilon}-\lambda g_{x_{i}}^{\varepsilon} \leq 0$, we see (using an approximation argument) that $u_{x_{1}}^{\varepsilon}-\lambda u_{x_{i}}^{\varepsilon} \geq 0$, for every $i$. Analogously, it follows that $u_{x_{1}}^{\varepsilon}+\lambda u_{x_{i}}^{\varepsilon} \geq 0$. Thus, if $\varepsilon_{j} \rightarrow 0$ and $u=\lim u^{\varepsilon_{j}}$, we obtain,

$$
u_{t} \leq 0 \quad \text { and } \quad u_{x_{1}}-\frac{N}{\lambda}|\nabla u| \geq 0 \quad \text { in }\{u>0\} .
$$

In particular, the free boundary is Lipschitz in space.

Next we want to apply Theorem 9.3 to show that $\bar{u}$ is a viscosity solution to $P(f)$ in $\mathbb{R}^{N} \times(0, T)$.

We need to show that $\bar{u}$ is nondegenerate at every point $\left(x_{0}, t_{0}\right) \in \partial\{\bar{u}>0\}$ which is regular from the left. In fact, $\left\{\bar{u}\left(\cdot, t_{0}\right)>0\right\}=\left\{x_{1}>\phi\left(x^{\prime}\right)\right\}$ with $\phi$ Lipschitz and, since $\bar{u}_{t} \leq 0$, then $\left\{(x, t) / x_{1}>\phi\left(x^{\prime}\right), t \leq t_{0}\right\} \subset\{\bar{u}>0\}$. Therefore, if we show that for some $\tau>0$ small

$$
\frac{\bar{u}^{\varepsilon_{j}}}{\varepsilon_{j}} \rightarrow 0 \quad \text { a. e. in }\{\bar{u} \equiv 0\}^{\circ} \cap Q_{\tau}^{-}\left(x_{0}, t_{0}\right)
$$

then, $\bar{u}$ will be under the hypotheses of Theorem 6.2 at $\left(x_{0}, t_{0}\right)$ and the nondegeneracy of $\bar{u}$ at $\left(x_{0}, t_{0}\right)$ will follow.

In order to show (9.6) we will prove that

$$
B_{\varepsilon}\left(\bar{u}^{\varepsilon}\right) \rightarrow M \chi\{\bar{u}>0\} \quad \text { in } L_{\mathrm{loc}}^{1}\left(Q_{\tau}^{-}\left(x_{0}, t_{0}\right)\right)
$$

(where for simplicity we remove the subscript $j$ ). Thus (9.7) will give $B\left(\frac{\bar{u}^{\varepsilon}}{\varepsilon}\right)=B_{\varepsilon}\left(\bar{u}^{\varepsilon}\right) \rightarrow 0$ a.e. in $\{\bar{u} \equiv 0\}^{\circ}$, which implies $(9.6)$.

It remains to prove that (9.7) holds. In fact, from Lemma 5.1 we know that $B_{\varepsilon}\left(\bar{u}^{\varepsilon}\right) \rightarrow \bar{M}$, in $L_{\text {loc }}^{1}$, with $\bar{M}(x, t)=0$ or $\bar{M}(x, t)=M$ almost everywhere in $\mathbb{R}^{N} \times(0, T)$. We also know that $\bar{M}(x, t)=M$ in $\{\bar{u}>0\}$. On the other hand, there holds that

$$
\left(\frac{\partial}{\partial x_{1}} \pm \lambda \frac{\partial}{\partial x_{i}}\right) B_{\varepsilon}\left(\bar{u}^{\varepsilon}\right)=\beta_{\varepsilon}\left(\bar{u}^{\varepsilon}\right)\left(\frac{\partial}{\partial x_{1}} \pm \lambda \frac{\partial}{\partial x_{i}}\right) \bar{u}^{\varepsilon} \geq 0,
$$

implying that $B_{\varepsilon}\left(\bar{u}^{\varepsilon}\right)(\cdot, t)$ is monotone in the spatial cone $\Gamma$ with axis $e_{1}$, for every $t$.

Now let $\left(\tilde{x}_{0}, \tilde{t}_{0}\right) \in Q_{\tau}^{-}\left(x_{0}, t_{0}\right) \cap \partial\{\bar{u}>0\}$. Let us suppose that there exists $r>0$ such that

$$
\bar{M}\left(x, \tilde{t}_{0}\right) \equiv M \quad \text { in } B_{r}\left(\tilde{x}_{0}\right) .
$$

Since $\frac{\partial}{\partial t} B_{\varepsilon}\left(\bar{u}^{\varepsilon}\right)=\beta_{\varepsilon}\left(\bar{u}^{\varepsilon}\right) \frac{\partial \bar{u}^{\varepsilon}}{\partial t} \leq 0$, then $\bar{M}$ is nonincreasing in $t$ and thus, $\bar{M}(x, t) \equiv M$ in $B_{r}\left(\tilde{x}_{0}\right) \times\left[0, \tilde{t}_{0}\right]$.

Let $y_{0} \in B_{r / 2}\left(\tilde{x}_{0}\right)$ be such that $\bar{u}\left(y_{0}, \tilde{t}_{0}\right)>0$. Let $d=\operatorname{dist}\left(y_{0}, \partial\left\{\bar{u}\left(\cdot, \tilde{t}_{0}\right)>0\right\}\right)$ and let $z_{0}$ be a point where the distance is attained. Then, since $\bar{u}_{t} \leq 0$, there holds that $B_{d}\left(y_{0}\right) \times\left[0, \tilde{t}_{0}\right] \subset$ $\{\bar{u}>0\}$. This is, $\left(z_{0}, \tilde{t}_{0}\right) \in \partial\{\bar{u}>0\}$ and it is a regular point from the right.

Taking $\bar{u}_{\lambda}(x, t)=\frac{1}{\lambda} \bar{u}\left(\lambda x+z_{0}, \lambda^{2} t+\tilde{t}_{0}\right)$ we get, for a sequence $\lambda_{k} \rightarrow 0$, that $\bar{u}_{\lambda_{k}} \rightarrow \bar{u}_{0}$ uniformly on compacts. If $\nu=\frac{y_{0}-z_{0}}{\left|y_{0}-z_{0}\right|}$, Lemma A.1 and Hopf's Principle (see Corollary 9.1) give

$$
\bar{u}_{0}(x, t)=\alpha\langle x, \nu\rangle^{+} \quad \text { in }\langle x, \nu\rangle \geq 0, t \leq 0,
$$

for some $\alpha>0$, and Corollary A.1 in [7] yields

$$
\bar{u}_{0}(x, t)=\beta\langle x, \nu\rangle^{-}+o\left(|x|+|t|^{\frac{1}{2}}\right) \quad \text { in }\langle x, \nu\rangle \leq 0, t \leq 0,
$$


with $\beta \geq 0$. Then, defining $\left(\bar{u}_{0}\right)_{\lambda}(x, t)=\frac{1}{\lambda} \bar{u}_{0}\left(\lambda x, \lambda^{2} t\right)$ we get, for a sequence $\lambda_{k}^{\prime} \rightarrow 0$, that $\left(\bar{u}_{0}\right)_{\lambda_{k}^{\prime}} \rightarrow \bar{u}_{00}$ with

$$
\bar{u}_{00}(x, t)=\alpha\langle x, \nu\rangle^{+}+\beta\langle x, \nu\rangle^{-} \quad \text { in } t \leq 0 .
$$

Now the monotonicity of $\bar{u}(\cdot, t)$ in the directions of the cone $\Gamma$ implies $\beta=0$.

On the other hand, proceeding as in [8], Theorem 3.1, we get, for every $\psi \in C_{0}^{\infty}\left(\mathbb{R}^{N} \times(-\infty, 0)\right)$,

$$
\begin{aligned}
\iint\left(\bar{u}_{\lambda}\right)_{t}\left(\bar{u}_{\lambda}\right)_{x_{1}} \psi & =\frac{1}{2} \iint\left|\nabla \bar{u}_{\lambda}\right|^{2} \psi_{x_{1}}-\iint\left(\bar{u}_{\lambda}\right)_{x_{1}} \nabla \bar{u}_{\lambda} \nabla \psi \\
& +M \iint \psi_{x_{1}}-\lambda \iint f\left(z_{0}+\lambda x, \tilde{t}_{0}+\lambda^{2} t\right)\left(\bar{u}_{\lambda}\right)_{x_{1}} \psi .
\end{aligned}
$$

Therefore, as $\lambda_{k} \rightarrow 0$, we obtain

$$
\iint\left(\bar{u}_{0}\right)_{t}\left(\bar{u}_{0}\right)_{x_{1}} \psi=\frac{1}{2} \iint\left|\nabla \bar{u}_{0}\right|^{2} \psi_{x_{1}}-\iint\left(\bar{u}_{0}\right)_{x_{1}} \nabla \bar{u}_{0} \nabla \psi
$$

It is not hard to see that (9.9) also holds with $\bar{u}_{0}$ replaced by $\bar{u}_{00}$. In fact, we obtain so if we take test functions of the form $\psi\left(\frac{x}{\lambda}, \frac{t}{\lambda^{2}}\right)$, we rescale and then let $\lambda_{k}^{\prime} \rightarrow 0$. Now using that $\bar{u}_{00}(x, t)=\alpha\langle x, \nu\rangle^{+}$in $t \leq 0$, we get that $\alpha=0$, a contradiction.

Thus (9.8) is not possible at a point $\left(\tilde{x}_{0}, \tilde{t}_{0}\right) \in Q_{\tau}^{-}\left(x_{0}, t_{0}\right) \cap \partial\{\bar{u}>0\}$. Therefore, using the monotonicity of $\bar{M}(\cdot, t)$ and $\bar{u}(\cdot, t)$ in the directions of $\Gamma$ we conclude that (9.7) holds. The proof is complete.

\section{The SYSTEM OF EQUATIONS IN NONLOCAL COMBUSTION}

In this section we consider the following system of equations

$$
\left\{\begin{array}{l}
\theta \Delta u^{\varepsilon}+(1-\theta)\left(J * u^{\varepsilon}-u^{\varepsilon}\right)-u_{t}^{\varepsilon}=v^{\varepsilon} F_{\varepsilon}\left(u^{\varepsilon}\right)+g_{1}^{\varepsilon} \\
\theta \Delta v^{\varepsilon}+(1-\theta)\left(J * v^{\varepsilon}-v^{\varepsilon}\right)-v_{t}^{\varepsilon}=v^{\varepsilon} F_{\varepsilon}\left(u^{\varepsilon}\right)+g_{2}^{\varepsilon}
\end{array}\right.
$$

where $F_{\varepsilon}(s)=\frac{1}{\varepsilon^{2}} F\left(\frac{s}{\varepsilon}\right)$ with $F$ Lipschitz, $F>0$ in $(-\infty, 1), F \equiv 0$ in $[1,+\infty), g_{1}^{\varepsilon}, g_{2}^{\varepsilon} \in$ $L^{\infty}\left(\mathbb{R}^{n} \times(0, T)\right)$.

System (10.1) is the nonlocal, nonhomogenous counterpart of the system

$$
\left\{\begin{array}{l}
\Delta u^{\varepsilon}-u_{t}^{\varepsilon}=v^{\varepsilon} F_{\varepsilon}\left(u^{\varepsilon}\right) \\
\Delta v^{\varepsilon}-v_{t}^{\varepsilon}=v^{\varepsilon} F_{\varepsilon}\left(u^{\varepsilon}\right)
\end{array}\right.
$$

that was considered in [10]. This system appears in the propagation of premixed flames when both thermal and reactant diffusivities coincide. In this setting, $v^{\varepsilon}$ is the mass fraction of the reactant (and therefore $v^{\varepsilon} \geq 0$ ) and $u^{\varepsilon}$ is the rescaled temperature (actually, $u^{\varepsilon}=\lambda\left(T_{f}-T\right.$ ) where $T$ is the temperature of the gas and $T_{f}$ is the flame temperature).

When the initial data for (10.2) satisfy that $u_{0}^{\varepsilon}=v_{0}^{\varepsilon}$, (10.2) reduces to

$$
\Delta u^{\varepsilon}-u_{t}^{\varepsilon}=\beta_{\varepsilon}\left(u^{\varepsilon}\right)
$$

where $\beta_{\varepsilon}(s)=\frac{1}{\varepsilon} \beta\left(\frac{s}{\varepsilon}\right)$ with $\beta(s)=s F(s)$. Since in this case $u^{\varepsilon}=v^{\varepsilon} \geq 0$ we may assume that $\beta(s)=0$ for $s<0$.

Analogously, when the initial data and forcing terms for (10.1) satisfy that $u_{0}^{\varepsilon}=v_{0}^{\varepsilon}$ and $g_{1}^{\varepsilon}=g_{2}^{\varepsilon}$, (10.1) reduces to the equation

$$
\theta \Delta u^{\varepsilon}+(1-\theta)\left(J * u^{\varepsilon}-u^{\varepsilon}\right)-u_{t}^{\varepsilon}=\beta_{\varepsilon}\left(u^{\varepsilon}\right)+g_{1}^{\varepsilon}
$$

that has been considered throughout the paper. 
As in [10] we can study system (10.1) under the hypotheses that

(1) $v_{0}^{\varepsilon} \geq 0$.

(2) $\left\|\frac{v_{0}^{\bar{\varepsilon}}-u_{0}^{\varepsilon}}{\varepsilon}\right\|_{L^{\infty}\left(\mathbb{R}^{N}\right)} \leq C$ independently of $\varepsilon$ and $\frac{v_{0}^{\varepsilon}-u_{0}^{\varepsilon}}{\varepsilon} \rightarrow w_{0}(x)$ uniformly on compact sets of $\mathbb{R}^{N}$ with $w_{0} \geq-1$.

(3) $\left\|u_{0}^{\varepsilon}\right\|_{L^{\infty}\left(\mathbb{R}^{N}\right)} \leq C$ independently of $\varepsilon$ and $u_{0}^{\varepsilon} \rightarrow u_{0}$ uniformly on compact sets of $\mathbb{R}^{N}$.

In the present situation we add the following conditions on the data: $u_{0}^{\varepsilon}, v_{0}^{\varepsilon} \in C\left(\mathbb{R}^{N}\right)$ and

(1) $g_{2}^{\varepsilon} \leq 0$.

(2) $\left\|\frac{g_{1}^{\varepsilon}-g_{2}^{\varepsilon}}{\varepsilon}\right\|_{L^{\infty}\left(\mathbb{R}^{N} \times(0, T)\right)} \leq C$ independently of $\varepsilon$ and $\frac{g_{1}^{\varepsilon}-g_{2}^{\varepsilon}}{\varepsilon} \rightarrow g_{0} *$-weakly in $L^{\infty}\left(\mathbb{R}^{N} \times\right.$ $(0, T))$.

(3) $\left\|g_{1}^{\varepsilon}\right\|_{L^{\infty}\left(\mathbb{R}^{N}\right)} \leq C$ independently of $\varepsilon$ and $g_{1}^{\varepsilon} \rightarrow g *$-weakly in $L^{\infty}\left(\mathbb{R}^{N} \times(0, T)\right)$.

If we let $w^{\varepsilon}=v^{\varepsilon}-u^{\varepsilon}, w^{\varepsilon}$ is a solution to

$$
\left\{\begin{array}{l}
\theta \Delta w^{\varepsilon}+(1-\theta)\left(J * w^{\varepsilon}-w^{\varepsilon}\right)-w_{t}^{\varepsilon}=g_{2}^{\varepsilon}-g_{1}^{\varepsilon}, \\
w^{\varepsilon}(x, 0)=v_{0}^{\varepsilon}(x)-u_{0}^{\varepsilon}(x)
\end{array}\right.
$$

By the results of Section 2 there exists a unique bounded solution $w^{\varepsilon}$ of (10.3) and a constant $C$ independent of $\varepsilon$ such that $\left\|w^{\varepsilon}\right\|_{L^{\infty}\left(\mathbb{R}^{N} \times(0, T)\right)} \leq C \varepsilon$. Thus, by standard local estimates for solutions of the nonhomogenous heat equation, for any open set $\mathcal{D} \subset \subset \mathbb{R}^{N} \times(0, T]$ there is a constant $C_{\mathcal{D}}$ such that

$$
\left\|w_{t}^{\varepsilon}\right\|_{L^{2}(\mathcal{D})} \leq C_{\mathcal{D}} \varepsilon, \quad\left\|\nabla w^{\varepsilon}\right\|_{L^{\infty}(\mathcal{D})} \leq C_{\mathcal{D}} \varepsilon
$$

We have that $u^{\varepsilon}$ is a solution to

$$
\left\{\begin{array}{l}
\theta \Delta u^{\varepsilon}+(1-\theta)\left(J * u^{\varepsilon}-u^{\varepsilon}\right)-u_{t}^{\varepsilon}=\left(u^{\varepsilon}+w^{\varepsilon}(x, t)\right) F_{\varepsilon}\left(u^{\varepsilon}\right)+g_{1}^{\varepsilon} \\
u^{\varepsilon}(x, 0)=u_{0}^{\varepsilon}(x)
\end{array}\right.
$$

As in Section 2 , we can prove that there exists a unique bounded solution to (10.5) with $\left\|u^{\varepsilon}\right\|_{L^{\infty}\left(\mathbb{R}^{N} \times(0, T)\right)} \leq C$ and $C$ independent of $\varepsilon$. Moreover, $u^{\varepsilon}=u^{\varepsilon}+w^{\varepsilon}-w^{\varepsilon} \geq-w^{\varepsilon} \geq-C \varepsilon$ since $u^{\varepsilon}+w^{\varepsilon}=v^{\varepsilon}$ is a bounded solution to

$$
\left\{\begin{array}{l}
\theta \Delta v^{\varepsilon}+(1-\theta)\left(J * v^{\varepsilon}-v^{\varepsilon}\right)-v_{t}^{\varepsilon}=v^{\varepsilon} F_{\varepsilon}\left(u^{\varepsilon}\right)+g_{2}^{\varepsilon}, \\
v^{\varepsilon}(x, 0)=v_{0}^{\varepsilon}(x) \geq 0 .
\end{array}\right.
$$

So that $v^{\varepsilon} \geq 0$ in $\mathbb{R}^{N} \times(0, T)$. Thus, $u^{\varepsilon}$ are uniformly bounded solutions to

$$
\theta \Delta u^{\varepsilon}-u_{t}^{\varepsilon}=\left(u^{\varepsilon}+w^{\varepsilon}(x, t)\right) F_{\varepsilon}\left(u^{\varepsilon}\right)+f^{\varepsilon}
$$

where $f^{\varepsilon}=g_{1}^{\varepsilon}-(1-\theta)\left(J * u^{\varepsilon}-u^{\varepsilon}\right)$ are uniformly bounded.

We can therefore consider the following local situation: $u^{\varepsilon}$ are uniformly bounded solutions to (10.7) in an open set $\mathcal{D}$ such that

i) $u^{\varepsilon} \geq-C \varepsilon$,

ii) $\frac{w^{\varepsilon}(x, t)}{\varepsilon} \rightarrow w_{0}(x, t)$ uniformly with $w_{0} \geq-1$ and $w_{0}$ bounded,

iii) $w^{\varepsilon}$ satisfy the estimates (10.4),

iv) $f^{\varepsilon}$ uniformly bounded and $f^{\varepsilon} \rightarrow f *$ - weakly in $L^{\infty}(\mathcal{D})$.

As in [10] and Section 4 in this paper we deduce that there exists $L$ independent of $\varepsilon$ such that, on compact subsets,

$$
\left|\nabla u^{\varepsilon}\right| \leq L \quad\left|u^{\varepsilon}(x, t+\Delta t)-u^{\varepsilon}(x, t)\right| \leq L \Delta t^{1 / 2} .
$$


Now, we deduce results similar to those of Sections 4 to 9 for (10.7) with the constant $M$ replaced by the function

$$
M(x, t)=\int_{-w_{0}(x, t)}^{1}\left(s+w_{0}(x, t)\right) F(s) d s
$$

In the global situation $w_{0}(x, t)=\lim _{\varepsilon \rightarrow 0} \frac{w^{\varepsilon}(x, t)}{\varepsilon}$ is the unique bounded solution to

$$
\left\{\begin{array}{l}
\theta \Delta w_{0}+(1-\theta)\left(J * w_{0}-w_{0}\right)-w_{0 t}=g_{0} \\
w_{0}(x, 0)=w_{0}(x) .
\end{array}\right.
$$

In the statements and proofs we consider the function $B_{\varepsilon}(z, x, t)=\int_{-w^{\varepsilon}(x, t)}^{z}\left(s+w^{\varepsilon}(x, t)\right) F_{\varepsilon}(s) d s$ instead of the function $B_{\varepsilon}(z)=\int_{0}^{z} \beta_{\varepsilon}(s) d s$, and we use the bounds (10.4) and the fact that $\left(u^{\varepsilon}+w^{\varepsilon}\right) F_{\varepsilon}\left(u^{\varepsilon}\right)$ is locally bounded in $L^{1}$ norm. We refer to [10] for details.

\section{A. Appendix}

In this section we prove some auxiliary results on the asymptotic behavior of nonnegative caloric functions at regular boundary points from the right or left (see Definition 8.1). These results are, in a sense, a generalization of Lemma A.1 of [5] and Lemma A.1 of [7]. (See also the related result, Lemma 2.1 in [1]). We will need some notation:

$$
\begin{aligned}
& Q_{\tau}=Q_{\tau}(0,0)=\left\{(x, t) / \max \left\{|x|,|t|^{\frac{1}{2}}\right\}<\tau\right\}, \quad Q_{\tau}^{-}=Q_{\tau} \cap\{t \leq 0\}, \\
& D_{\tau}=Q_{\tau}^{-} \cap B_{r}\left(x_{0}, t_{0}\right), \quad D_{1}^{k}=Q_{1}^{-} \cap\left\{x_{1}>F_{k}\left(x^{\prime}, t\right)\right\}, \quad \mathcal{B}_{\tau}=Q_{\tau}^{-} \cap\left\{x_{1}>0\right\},
\end{aligned}
$$

for fixed $r>0$ and $\left(x_{0}, t_{0}\right)$. Here we have denoted a point $x$ in $\mathbb{R}^{N}$ by $x=\left(x_{1}, x^{\prime}\right)$ with $x^{\prime} \in \mathbb{R}^{N-1}$ and $F_{k}$ is a function that will be defined later on.

Lemma A.1. Let $u \in \operatorname{Lip}(1,1 / 2)$ in $\overline{Q_{\tau}^{-}}(0,0)$, for some $\tau>0$. Assume there exists a ball $B=B_{r}\left(x_{0}, t_{0}\right) \subset \subset Q_{\tau}(0,0)$, with $(0,0) \in \partial B$ and $e_{1}$ the inward unit spatial normal to $\partial B$ at $(0,0)$, such that $u$ is positive and $\Delta u-u_{t} \leq C$ in $B \cap\{t \leq 0\}$, and that $u(0,0)=0$. Then, $u$ has the asymptotic development

$$
u(x, t)=\bar{\alpha} x_{1}+o\left(|x|+|t|^{\frac{1}{2}}\right), \quad \text { in } B \cap\{t \leq 0\}
$$

with $\bar{\alpha} \geq 0$.

Proof. The proof is a modification of the proof of [7], Lemma A.1. Without loss of generality we may assume that $\tau=1$ and $r>>1$. Let $v>0$ in $D_{1}, \Delta v-v_{t}=0$ in $D_{1}$ with $v=0$ in $\partial_{p} D_{1} \cap Q_{1}^{-}$. For each $k \in \mathbb{N}$ let

$$
\varepsilon_{k}=\sup \left\{\varepsilon / u(x, t) \geq \varepsilon v(x, t) \text { in } D_{2^{-k}}\right\} .
$$

Let $\alpha=\lim _{k \rightarrow \infty} \varepsilon_{k}$ (observe that $\varepsilon_{k} \uparrow$ ). Then, $\alpha<\infty$. In fact, by Hopf Lemma

$$
v(x, t) \geq \bar{\gamma} x_{1} \quad \text { in } D_{\nu}
$$

for some $\bar{\gamma}>0, \nu>0$. On the other hand, since $u \in \operatorname{Lip}(1,1 / 2)$ in $Q_{\tau}^{-}$,

$$
u(x, t) \leq K\left(|x|+|t|^{\frac{1}{2}}\right) .
$$

Thus, for $k$ large enough, $\varepsilon_{k} \leq \frac{K}{\bar{\gamma}}$. We claim that,

$$
u(x, t) \geq \alpha v(x, t)+o\left(|x|+|t|^{\frac{1}{2}}\right) .
$$


In fact, since $v$ is a smooth function, $v(0,0)=0$, for $(x, t) \in D_{2^{-k}}$

$$
u(x, t)-\alpha v(x, t) \geq-\left(\alpha-\varepsilon_{k}\right) v(x, t) \geq-\left(\alpha-\varepsilon_{k}\right) L\left(|x|+|t|^{\frac{1}{2}}\right),
$$

where $L$ is the Lipschitz constant of $v$. Let us see that equality holds in (A.1). If equality does not hold, there exists $\delta_{0}>0$ and a sequence $\left(x_{k}, t_{k}\right) \rightarrow 0$ such that

$$
u\left(x_{k}, t_{k}\right)-\alpha v\left(x_{k}, t_{k}\right) \geq \delta_{0} \max \left\{\left|x_{k}\right|,\left|t_{k}\right|^{\frac{1}{2}}\right\} .
$$

Let $r_{k}=\max \left\{\left|x_{k}\right|,\left|t_{k}\right|^{\frac{1}{2}}\right\} \rightarrow 0, u_{k}(x, t)=\frac{1}{r_{k}} u\left(r_{k} x, r_{k}^{2} t\right), v_{k}(x, t)=\frac{1}{r_{k}} v\left(r_{k} x, r_{k}^{2} t\right)$. Then, by (A.1)

$$
u_{k}(x, t)-\alpha v_{k}(x, t) \geq o(1) \quad \text { in } D_{1}^{k}
$$

where the function $F_{k}$ in the definition of $D_{1}^{k}$ is $F_{k}\left(x^{\prime}, t\right)=\frac{1}{r_{k}} F\left(r_{k} x^{\prime}, r_{k}^{2} t\right)$ and $x_{1}=F\left(x^{\prime}, t\right)$ is the equation of the boundary of the ball $B=B_{r}\left(x_{0}, t_{0}\right)$ in a neighborhood of $(0,0)$. So that, since $F \in C^{\infty}, F(0,0)=0$ and $\nabla_{x^{\prime}} F(0,0)=0$ then, in $\left\{\max \left\{\left|x^{\prime}\right|,|t|^{\frac{1}{2}}\right\}<1\right\}$,

$$
F_{k} \rightrightarrows 0, \quad \nabla_{x^{\prime}} F_{k} \rightrightarrows 0, \quad \frac{\partial}{\partial t} F_{k} \rightrightarrows 0 .
$$

Since $u_{k}, v_{k} \in \operatorname{Lip}(1,1 / 2)$ in $D_{1}^{k}$ with seminorm uniformly bounded with respect to $k, u_{k}(0,0)=$ $v_{k}(0,0)=0, D_{1}^{k} \subset D_{1}^{k+1}, \bigcup_{k} D_{1}^{k}=\mathcal{B}_{1}$, there exist $U$ and $V$ such that $u_{k} \rightarrow U, v_{k} \rightarrow V$ in $\mathcal{B}_{1}$. Actually, $u_{k} \rightrightarrows U$ in $\mathcal{B}_{1}$ since $u \in \operatorname{Lip}(1,1 / 2)$ in $Q_{1}^{-}$. There holds that, $U-\alpha V \geq 0$ in $\mathcal{B}_{1}$. On the other hand, $\Delta U-U_{t} \leq 0$ since $\Delta u_{k}-u_{k t} \leq r_{k} C . \Delta V-V_{t}=0$ since this is true for $v_{k}$. Let $\left(\bar{x}_{k}, \bar{t}_{k}\right)=\left(\frac{x_{k}}{r_{k}}, \frac{t_{k}}{r_{k}^{2}}\right)$. Then,

$$
\max \left\{\left|\bar{x}_{k}\right|,\left|\bar{t}_{k}\right|^{1 / 2}\right\}=1 \quad \text { and } \quad u_{k}\left(\bar{x}_{k}, \bar{t}_{k}\right)-\alpha v_{k}\left(\bar{x}_{k}, \bar{t}_{k}\right) \geq \delta_{0} .
$$

Thus, we may assume that $\left(\bar{x}_{k}, \bar{t}_{k}\right) \rightarrow(\bar{x}, \bar{t})$ with $(\bar{x}, \bar{t}) \in \partial_{p} Q_{1}^{-}$. Also, we may assume that $\bar{t}<0$ and $\bar{x}_{1}>0$ and that there exists $\varepsilon>0$ such that, $u_{k}(x, t)-\alpha v_{k}(x, t) \geq \frac{\delta_{o}}{2}$ in $B_{\varepsilon}(\bar{x}, \bar{t})$. Let $w_{k}$ be the solution to

$$
\begin{cases}\Delta w_{k}-w_{k t}=r_{k} C & \text { in } D_{1}^{k} \\ w_{k}=\min \left\{0, u_{k}-\alpha v_{k}\right\} & \text { on } \partial_{p} D_{1}^{k} \backslash B_{\varepsilon}(\bar{x}, \bar{t}) \\ w_{k}=\delta_{0} / 4 & \text { on } \partial_{p} D_{1}^{k} \cap B_{\varepsilon / 2}(\bar{x}, \bar{t}) \\ 0 \leq w_{k} \leq \delta_{0} / 4 & \text { on } \partial_{p} D_{1}^{k} \cap B_{\varepsilon}(\bar{x}, \bar{t})\end{cases}
$$

with $w_{k}$ smooth on $\partial_{p} D_{1}^{k}$ with uniformly bounded $\operatorname{Lip}(1,1 / 2)$ norm. Then, $u_{k}-\alpha v_{k} \geq w_{k}$ in $D_{1}^{k}$. Let us see that,

$$
w_{k}(x, t) \geq \gamma\left(x_{1}-F_{k}\left(x^{\prime}, t\right)\right) \quad \text { in } D_{1}^{k} \cap B_{\nu}(0,0)
$$

for some $\gamma>0, \nu>0$. To this end let us consider the application

$$
y_{1}=x_{1}-\chi\left(x_{1}\right) F_{k}\left(x^{\prime}, t\right), \quad y^{\prime}=x^{\prime}, \quad t=t
$$

where $\chi \in C^{\infty}(\mathbb{R})$ is such that $\chi(s)=1$ if $s<\bar{x}_{1} / 3, \chi(s)=0$ if $s>2 \bar{x}_{1} / 3$. Then, for $k$ large enough, this application is one to one between $D_{1}^{k}$ and $\mathcal{B}_{1}$. For $(y, t) \in \mathcal{B}_{1}$, let $\bar{w}_{k}(y, t)=w_{k}(x, t)$. Then,

$$
\begin{gathered}
\Delta w_{k}-w_{k t}=\left[\left(1-\chi^{\prime} F_{k}\right)^{2}-\chi^{2}\left|\nabla F_{k}\right|^{2}\right] \frac{\partial^{2} \bar{w}_{k}}{\partial y_{1}^{2}}+\Delta_{y^{\prime}} \bar{w}_{k}-2 \sum_{i=2}^{N} \chi \frac{\partial F_{k}}{\partial x_{i}} \frac{\partial^{2} \bar{w}_{k}}{\partial y_{1} \partial y_{i}}-\frac{\partial \bar{w}_{k}}{\partial t} \\
-\frac{\partial \bar{w}_{k}}{\partial y_{1}}\left(\chi^{\prime \prime} F_{k}+\chi\left(\Delta_{x^{\prime}} F_{k}-F_{k t}\right)\right)=L_{k} \bar{w}_{k} .
\end{gathered}
$$


Thus,

$$
L_{k} \bar{w}_{k}=r_{k} C \quad \text { in } \mathcal{B}_{1} .
$$

Since $F_{k} \rightrightarrows 0, \nabla F_{k} \rightrightarrows 0, L_{k}$ is uniformly parabolic with parabolicity constants and coefficient bounds independent of $k$. Moreover, as also $F_{k t} \rightrightarrows 0, L_{k} \rightarrow(\Delta-\partial / \partial t)$ as $k \rightarrow \infty$.

Moreover, $\bar{w}_{k}$ are uniformly bounded in $\operatorname{Lip}(1,1 / 2)$ norm on $\partial_{p} \mathcal{B}_{1}$. Thus, there exists a function $w \in C\left(\overline{\mathcal{B}}_{1}\right)$ such that $\bar{w}_{k} \rightrightarrows w$ in $\mathcal{B}_{1}$. Since $\bar{w}_{k} \geq o(1)$ on $\partial_{p} \mathcal{B}_{1}, \bar{w}_{k}=0$ on $\left\{x_{1}=0\right\}$, $\bar{w}_{k} \geq \delta_{0} / 4$ on $\partial_{p} \mathcal{B}_{1} \cap B_{\varepsilon / 2}(\bar{x}, \bar{t})$ the function $w$ satisfies,

$$
\begin{cases}\Delta w-w_{t}=0 & \text { in } \mathcal{B}_{1} \\ w \geq 0 & \text { on } \partial_{p} \mathcal{B}_{1} \\ w=0 & \text { on }\left\{x_{1}=0\right\} \\ w \geq \delta_{0} / 4 & \text { on } \partial_{p} \mathcal{B}_{1} \cap B_{\varepsilon / 2}(\bar{x}, \bar{t})\end{cases}
$$

so that $w>0$ in $\mathcal{B}_{1}$. Moreover, $w_{k} \rightarrow w$ in $\mathcal{B}_{1}$.

Let $\mu>0, \nu>0$ such that $w(x, t) \geq \mu x_{1}$ in $\mathcal{B}_{1} \cap B_{\nu}(0,0)$. Let us see that for $k$ large,

$$
\bar{w}_{k}(x, t) \geq \frac{\mu}{2} x_{1} \quad \text { in } \mathcal{B}_{1} \cap B_{\nu}(0,0) .
$$

In fact, by standard regularity results, as $w$ is caloric in $\mathcal{B}_{1}$ and $w=0$ on $\left\{x_{1}=0\right\}$, there holds that $\|w\|_{C^{2+\alpha, 1+\frac{\alpha}{2}}\left(\overline{\mathcal{B}}_{1 / 2}\right)} \leq \bar{C}$. Therefore, for every $\delta>0$ there exists $k_{0}$ such that $\left|L_{k} w\right| \leq \delta$ if $k \geq$ $k_{0}$. So that, for $k$ large enough

$$
\left|L_{k}\left(\bar{w}_{k}-w\right)\right| \leq 2 \delta \quad \text { in } \mathcal{B}_{1 / 2}, \quad\left\|\bar{w}_{k}-w\right\|_{L^{\infty}\left(\mathcal{B}_{1 / 2}\right)} \leq \delta, \quad \bar{w}_{k}=w=0 \quad \text { on }\left\{x_{1}=0\right\} .
$$

Thus, there exists a constant $K$ independent of $k$ such that $\left\|\nabla\left(\bar{w}_{k}-w\right)\right\|_{L^{\infty}\left(\mathcal{B}_{1 / 4}\right)} \leq K \delta$. Which implies, in particular that for $k$ large enough

$$
\left|\bar{w}_{k}-w\right| \leq \frac{\mu}{2} x_{1} \quad \text { in } \mathcal{B}_{1 / 4} .
$$

So that, for $k$ large enough $\bar{w}_{k} \geq \frac{\mu}{2} x_{1}$ in $\mathcal{B}_{1} \cap B_{\nu}(0,0)$. Thus,

$$
w_{k}(x, t) \geq \frac{\mu}{2}\left(x_{1}-F_{k}\left(x^{\prime}, t\right)\right) \quad \text { in } D_{1}^{k} \cap B_{\nu / 2}(0,0) .
$$

Since $v$ is smooth and $v=0$ on $\left\{x_{1}=F\left(x^{\prime}, t\right)\right\}$, there holds that $v(x, t) \leq L\left(x_{1}-F\left(x^{\prime}, t\right)\right)$, where $L$ is the Lipschitz constant of $v$. Thus, if $k$ is large enough and $(x, t) \in D_{r_{k}}$,

$$
u(x, t)-\alpha v(x, t) \geq \frac{\mu}{2}\left(x_{1}-F\left(x^{\prime}, t\right)\right) \geq \frac{\mu}{2 L} v(x, t) .
$$

This implies that there exists $k_{0}$ such that

$$
u(x, t) \geq\left(\alpha+\frac{\mu}{2 L}\right) v(x, t) \text { in } D_{r_{k_{0}}} .
$$

Let $j$ large so that $2^{-j}<r_{k_{0}}$. Then, $D_{2^{-j}} \subset D_{r_{k_{0}}}$ and since $\alpha \geq \varepsilon_{j}$,

$$
u(x, t) \geq\left(\varepsilon_{j}+\frac{\mu}{2 L}\right) v(x, t) \quad \text { in } D_{2^{-j}} .
$$

This contradicts the definition of $\varepsilon_{j}$ and thus,

$$
u(x, t)=\alpha v(x, t)+o\left(|x|+|t|^{\frac{1}{2}}\right) \text { in } D_{1} .
$$

Since $v$ is smooth, $v>0$ and $v(0,0)=0$,

$$
v(x, t)=\bar{\gamma} x_{1}+o\left(|x|+|t|^{\frac{1}{2}}\right) \quad \text { in } D_{1}
$$


which implies that

$$
u(x, t)=\bar{\alpha} x_{1}+o\left(|x|+|t|^{\frac{1}{2}}\right) \quad \text { in } D_{1} .
$$

Since $u \geq 0, \bar{\alpha} \geq 0$. The result is proved.

Lemma A.2. Let $u \in \operatorname{Lip}(1,1 / 2)$ in $\overline{Q_{\tau}^{-}}(0,0)$, for some $\tau>0$. Assume that $u$ is nonnegative and $\Delta u-u_{t} \geq-C$ in $Q_{\tau}^{-}(0,0)$ and $u(0,0)=0$. Assume, in addition, that there exists a ball $B_{r}\left(x_{0}, t_{0}\right) \subset \subset Q_{\tau}(0,0)$, with $(0,0) \in \partial B_{r}\left(x_{0}, t_{0}\right)$ and $e_{1}$ the outward unit spatial normal to $\partial B_{r}\left(x_{0}, t_{0}\right)$ at $(0,0)$, such that $u \equiv 0$ in $B_{r}\left(x_{0}, t_{0}\right) \cap\{t \leq 0\}$. Then, $u$ has the asymptotic development

$$
u(x, t)=\beta x_{1}^{+}+o\left(|x|+|t|^{\frac{1}{2}}\right), \quad \text { in }\{t \leq 0\}
$$

with $\beta \geq 0$.

Proof. The proof follows by arguing as in Lemma 4.1 of [15], using in this case Lemma A.1 above, instead of Lemma A.1 of [5]. In fact, let $r^{\prime}>r$ such that $B_{r^{\prime}}\left(x_{0}, t_{0}\right) \cap\{t \leq 0\} \subset Q_{\tau}^{-}(0,0)$. Let $\kappa>0$ small and $\mathcal{D}=\left(B_{r^{\prime}}\left(x_{0}, t_{0}\right) \backslash \bar{B}_{r}\left(x_{0}, t_{0}\right)\right) \cap\{\kappa<t \leq 0\}$. Let $v$ be the solution to

$$
\begin{cases}\Delta v-v_{t}=-C & \text { in } \mathcal{D} \\ v=\Lambda & \text { on } \partial_{p} \mathcal{D} \backslash \partial B_{r}\left(x_{0}, t_{0}\right) \\ v=0 & \text { on } \partial_{p} \mathcal{D} \cap \partial B_{r}\left(x_{0}, t_{0}\right)\end{cases}
$$

where $\Lambda>0$ is an upper bound for $u$ in $Q_{\tau}^{-}(0,0)$. Then, $v$ is smooth in a neighborhood of the origin and therefore

$$
v(x, t)=\delta x_{1}+o\left(|x|+|t|^{\frac{1}{2}}\right) \quad \text { in } \mathcal{C} B_{r}\left(x_{0}, t_{0}\right) \cap\{t \leq 0\} .
$$

Now let $B:=B_{\rho}\left(-\frac{\rho}{r}\left(x_{0}, t_{0}\right)\right)$ with $\rho$ small enough so as to have $B \cap\{t \leq 0\} \subset \mathcal{D}$. Then,

$$
\begin{cases}\Delta(v-u)-(v-u)_{t} \leq 0 & \text { in } B \cap\{t \leq 0\} \\ v-u>0 & \text { in } B \cap\{t \leq 0\} \\ (v-u)(0,0)=0 . & \end{cases}
$$

Thus, by Lemma A.1,

$$
(v-u)(x, t)=\gamma x_{1}+o\left(|x|+|t|^{\frac{1}{2}}\right) \quad \text { in } B \cap\{t \leq 0\} .
$$

So that, by (A.4) and (A.5),

$$
u(x, t)=\beta x_{1}+o\left(|x|+|t|^{\frac{1}{2}}\right) \quad \text { in } \mathcal{C} B_{r}\left(x_{0}, t_{0}\right) \cap\{t \leq 0\} .
$$

Acknowledgements. The authors want to thank Prof. Henri Berestycki for a helpful discussion concerning this nonlocal model and to Prof. Panagiotis Souganidis for providing interesting references on nonlocal models. They also want to thank the referee for helpful comments that improved the presentation of the paper. 


\section{REFERENCES}

[1] I. Athanasopoulos, L. A. Caffarelli, S. Salsa, Phase transition problems of parabolic type: Flat free boundaries are smooth, Communications in Pure and Applied Mathematics 51(1), (1998), 77-112.

[2] H. Berestycki, L. A. Caffarelli, L. Nirenberg, Uniform estimates for regularization of free boundary problems, "Analysis and Partial Differential Equations" (Cora Sadosky, ed.), Lecture Notes in Pure and Applied Mathematics, vol. 122, Marcel Dekker, New York, 1990, 567-619.

[3] J. D. Buckmaster, G. S. S. Ludford, Theory of Laminar Flames, Cambridge University Press, Cambridge, 1982.

[4] J. Coville, Travelling wave in non-local reaction diffusion equation with ignition nonlinearity, preprint.

[5] L. A. Caffarelli, A Harnack inequality approach to the regularity of free boundaries. Part II: Flat free boundaries are Lipschitz, Comm. Pure and Appl. Math. 42 , (1989), 55-78.

[6] L. A. Caffarelli, Uniform Lipschitz Regularity of a Singular Perturbation Problem, Diff. Int. Eqs. 8(7), (1995), 1585-1590.

[7] L. A. Caffarelli, C. Lederman, N. Wolanski, Uniform estimates and limits for a two phase parabolic singular perturbation problem, Indiana Univ. Math. J. 46 (2), (1997), 453-490.

[8] L. A. Caffarelli, C. Lederman, N. Wolanski, Pointwise and viscosity solutions for the limit of a two phase parabolic singular perturbation problem, Indiana Univ. Math. J. 46 (3), (1997), 719-740.

[9] L. A. Caffarelli, J. L. Vazquez, A free boundary problem for the heat equation arising in flame propagation, Trans. Amer. Math. Soc. 347 (1995), 411-441.

[10] J. Fernández Bonder, N. Wolanski, A free boundary problem in combustion theory. Interfaces Free Bound., 2, (2000), 381-411.

[11] P. Fife. Some nonclassical trends in parabolic and parabolic-like evolutions. Trends in nonlinear analysis, 153-191, Springer, Berlin, 2003.

[12] L.I. Kamynin, B.N. Himcenko, On applications of the maximum principle to parabolic equations of second order, Soviet Math. Doklady 13(3) (1972), 683-686.

[13] O. Ladyzenskaja, V. Solonnikov, N. Ural'ceva, Linear and quasilinear equations of parabolic type, Translations of Math. Mon. Vol. 23, Amer. Math. Soc., 1988.

[14] C. Lederman, J. L. Vázquez, N. Wolanski, Uniqueness of solution to a free boundary problem from combustion, Transactions of the American Mathematical Society 353 (2), (2001), 655-692.

[15] C. Lederman, N. Wolanski, Viscosity solutions and regularity of the free boundary for the limit of an elliptic two phase singular perturbation problem, Annali della Scuola Normale Sup. Pisa, Cl. Sci., Serie IV 27 (2), (1998), 253-288.

[16] P. Souganidis, Recent developments in the theory of front propagation and its applications, Modern methods in scientific computing and applications (Montral, QC, 2001), 397-449, NATO Sci. Ser. II Math. Phys. Chem., 75, Kluwer Acad. Publ., Dordrecht, 2002.

[17] J. L. Vázquez, The free boundary problem for the heat equation with fixed gradient condition, Proceedings International Conference on free boundary problems and applications, Zakopane, Poland, 1995.

[18] G. S. Weiss, A singular limit arising in combustion theory: fine properties of the free boundary, Calculus of Variations and Partial Diff. Equations 17(3), (2003), 311-340.

[19] Ya.B. Zeldovich, D.A. Frank-Kamenetski, The theory of thermal propagation of flames, Zh. Fiz. Khim. 12, (1938), 100-105 (in Russian); English translation in "Collected Works of Ya.B. Zeldovich", vol. 1, Princeton Univ. Press, 1992. 